\title{
Competitive Rational Expectations Equilibria without Apology
}

\author{
ALEXANDER KOVALENKOV \\ XAVIER VIVES \\ CESIFO WORKING PAPER NO. 2446 \\ CATEgORY 6: MONETARY POLICY AND INTERNATIONAL FINANCE \\ NOVEMBER 2008
}

An electronic version of the paper may be downloaded
- from the SSRN website: $\quad$ www.SSRN.com
- from the RePEc website:
- from the CESifo website: 


\title{
Competitive Rational Expectations Equilibria without Apology
}

\begin{abstract}
In a standard financial market model with asymmetric information with a finite number $\mathrm{N}$ of risk-averse informed traders, competitive rational expectations equilibria provide a good approximation to strategic equilibria as long as $\mathrm{N}$ is not too small: equilibrium prices in each situation converge to each other at a rate of $1 / \mathrm{N}$ as the market becomes large. The approximation is particularly good when the informationally adjusted risk bearing capacity of traders is not very large. This is not the case if informed traders are close to risk neutral. Both equilibria converge to the competitive equilibrium of an idealized limit continuum economy as the market becomes large at a slower rate of $1 / \sqrt{ } \mathrm{N}$ and, therefore, the limit equilibrium need not be a good approximation of the strategic equilibrium in moderately large markets.
\end{abstract}

JEL Code: C22, G10, G12, D41, D43.

Keywords: "schizophrenia" problem, strategic equilibrium, large markets, information acquisition, free entry, rate of convergence.

\author{
Alexander Kovalenkov \\ University of Glasgow \\ Department of Economics \\ Adam Smith Building \\ UK - Glasgow G12 8RT \\ A.Kovalenkov@lbss.gla.ac.uk
}

\author{
Xavier Vives \\ Department of Economics \\ IESE Business School \\ University of Navarra \\ Avenida Pearson, 21 \\ Spain - 08034 Barcelona \\ xvives@iese.edu
}

Running Title: Competitive REE without apology

We are grateful for the helpful comments of the participants at the North American Winter Meeting of the Econometric Society in 2005 and at ESSFM 2008 in Gerzensee, in particular to Dimitri Vayanos and Paolo Vitale. Kovalenkov is grateful for support from the IBM Fund Award and the Latané Fund. Vives is grateful for support from Consolider 2010 (CSD200600016), and from project SEJ2005-08263 at UPF of the DGI of the Spanish Ministry of Education and Science. 


\section{Introduction}

The aim of this paper is to find out when we can safely use competitive rational expectations equilibria (REE) as an approximation of the "true" strategic equilibria in a standard financial market context. We would like to bound the error when approximating the equilibrium with a finite number of traders with a price-taking equilibrium, as is often done in applied work. The general result is that the competitive approximation basically works, even in a moderately sized market, when competitive traders have incentives to be restrained in their trading.

The concept of competitive REE has been questioned from different quarters. Hellwig (1980) pointed out the "schizophrenia" problem of price-taking behavior in a competitive REE with a finite number of traders. When submitting their demands traders would take into account the information content of the price but not the price impact of their trade. The problem disappears in a large market. Indeed, as the market becomes large the strategic equilibria of finite economies converge to the competitive REE of an idealized limit continuum economy (as described, e.g., by Admati (1985) or Vives (1995)). Kyle (1989) modeled the strategic equilibrium directly where traders are aware of the price impact of their trades and compete in demand schedules (in a REE with imperfect competition) and claimed that the properties of the imperfect competition model were reasonable.

In this paper we show, in the framework of Kyle's (1989) model, that the competitive REE of a large but finite market with risk-averse traders provides a good approximation of the "true" strategic equilibrium. This is particularly the case when the informationally adjusted risk-bearing capacity of traders is not very large. Then, if the market has a minimum size, we can take the shortcut of assuming competitive behavior of the finite number of agents present as a good enough approximation. That is, we can use competitive REE without apology. However, the shortcut does not work, for example, if informed traders are close to risk neutral. In any case the strategic equilibrium is not well approximated by the competitive REE of the idealized limit continuum economy.

Consider the imperfect competition model of Kyle (1989) with risk-averse speculators, parameterize the size of the market by the volume of noise trading $\sigma_{z}$ and let there be free entry of speculators. As in Verrecchia (1982) and Kyle (1989) speculators can become informed, acquiring a private signal of known precision, by paying a fixed cost, which may depend on the size of the market. The entry of uninformed speculators is free. We show first that, if the entry cost in a large market is positive but not too large, the equilibrium number of informed speculators $N$ is of the order of $\sigma_{z}$, that is, $N$ grows in proportion to $\sigma_{z}$, in both the strategic and competitive cases. We can then identify increases in the size of the market $\sigma_{z}$ with increases in $N$. This is our central scenario. The result is that the equilibrium prices in the strategic and competitive cases, as the market becomes large, converge to each other at a rate of $1 / N$, while both converge to the competitive equilibrium of the limit economy at a

rate of $1 / \sqrt{N}$. The same rates of convergence apply for demands, profits and relative utilities 
of traders. The consequence is that in moderately sized and large markets the assumption of competitive behavior with risk-averse traders turns out to be a good approximation to the "true" strategic (Bayesian) equilibrium. However, thinking in terms of the idealized continuum limit economy will not provide a good approximation for the equilibrium of the finite market. The point is that market power is dissipated quickly, at a rate of $1 / N$, while the distance between a finite and the limit economy depends on the rate at which the average error term in the signals of traders vanishes, and this is $1 / \sqrt{N}$.

The result that market power vanishes quickly at a rate of $1 / N$ as the market grows is consistent with the result obtained by Vives (2002) in the context of a Cournot model (which in the financial market would correspond to traders using market orders instead of demand schedules as in Kyle's (1989) model) and with Gong and McAfee (1996) for double auctions allowing correlated values (the discrepancy of the strategic equilibrium, Bayesian bidding equilibrium at the double auction, and the non-strategic price-taking equilibrium bid is of the order of $1 / N) .{ }^{1}$ Our results are also related to the literature that tries to provide a strategic foundation for competitive REE in a continuum economy. This is the aim, for example, of Reny and Perry (2006). They consider a double auction with a finite number of buyers and sellers with interdependent values and affiliated private information, and show that if there are sufficiently many buyers and sellers (and their bids are restricted to a sufficiently fine discrete set of prices), then generically there exists an equilibrium which is arbitrarily close to the unique fully revealing REE of the limit market. In particular, the double auction equilibrium is almost efficient in large economies (but they do not analyze convergence rates). In our model a large number of traders are needed for the equilibrium in the continuum economy to be a good approximation of the strategic equilibrium since the rate of convergence is $1 / \sqrt{N}$.

We also look at a more refined measure of convergence speed for a given rate of convergence: the asymptotic variance of the price difference in the different regimes. We find that the asymptotic variance of the price difference between the strategic and the competitive regime in a finite economy is small, and the approximation of the strategic equilibrium by the competitive equilibrium is good, when the prior volatility of the asset is low, noise trading is large in relation to the risk bearing capacity of the informed traders, or the signals are very noisy. ${ }^{2}$ We confirm, therefore, the idea that the competitive approximation works, even in a moderately sized market, basically when competitive traders have incentives to be restrained in their trading. As traders become less and less risk averse, the asymptotic

\footnotetext{
${ }^{1}$ The latter paper extends the work on independent and identically distributed (i.i.d.) double auction environments of Rustichini et al. (1994) to general information structures. Other extensions are provided by Cripps and Swinkels (2006). Yosha (1997) deals with financial intermediation in a large Cournot-Walras economy with i.i.d. shocks and computes the rates of convergence as the economy becomes large for various market parameters.

${ }^{2}$ However, while the asymptotic variance of the price difference between the strategic and the competitive regime is increasing in the volatility of fundamentals, it can be nonmonotonic in the volume of noise trading and the degree of risk aversion, and it is always nonmonotonic in the noisiness of the signals.
} 
variance of the price difference between the strategic and the competitive regime in a finite economy increases unboundedly, reflecting the fact that when traders are close to risk neutral, competitive prices are close to being fully revealing while prices at the strategic equilibrium are far from being so. This points to the fact that the competitive model is appropriate, as an approximation to the true strategic model, when traders are risk averse but not when they are close to risk neutral. In fact, when they are close to risk neutral and information is costly to acquire, no competitive traders would enter into the market and become informed because they would lose money (this is just a variant of the well-known Grossman and Stiglitz (1980) paradox). Indeed, the naive idea that with risk-neutral traders Bertrand competition would push the strategic and competitive regimes closer together does not hold. This is consistent with the results in Biais et al. (2000) according to which adverse selection softens supply schedule competition among risk-neutral market makers in a common value environment.

We also test the limits of our results by checking situations where the number of informed traders increases nonproportionally to the size of the market. If the number of informed traders increases faster than the size of the market, then a fully revealing equilibrium is obtained in the limit. If the number of informed traders increases more slowly than the size of the market, then an informationally trivial equilibrium is obtained in the limit. In all cases, market power is again dissipated faster than the rate at which the finite and the limit economy converge to each other as the market becomes large.

Finally, we look at a version of Kyle's (1989) "monopolistic competition case" where the competitive market need not be a good approximation of strategic trading even in a large market. This occurs when the cost of acquiring information tends to zero but the total precision of information for the informed is bounded. In this context traders retain some market power even in a large market. In this case convergence to the monopolistically competitive limit as the information purchase cost tends to zero occurs at most at a rate of $1 / N$. Therefore, our results on the approximation of strategic by competitive equilibria have to be qualified as holding in those situations where there is no residual market power in a large market.

The rest of the paper is organized as follows. In the next section we present the structure of the model and the equilibria that we are going to consider with a fixed number of informed speculators. In Section 3 we endogenize the number of informed speculators in a free entry model and provide a characterization of equilibria. The results on the speed of convergence to price-taking equilibria in the central scenario are presented in Section 4. Section 5 is devoted to the analysis of convergence in other large market scenarios. In section 6 we address traders who are almost risk neutral. In Section 7 we present the monopolistic competition case. Proofs of all the results are collected in the Appendix. 


\section{$2 \quad$ A market with $N$ informed speculators}

Consider Kyle's (1989) model (and to facilitate comparison we follow his notation as closely as possible). A single risky asset with random liquidation value $\tilde{v}$ is traded among noise traders, $N$ informed speculators, indexed $n=1, \ldots, N$, and market makers. ${ }^{3}$ The return to trade one unit at a market clearing price $\tilde{p}$ is thus $\tilde{v}-\tilde{p}$. Noise traders trade in the aggregate the random quantity $\tilde{z}$. Each informed speculator $n=1, \ldots, N$ receives a private signal $\tilde{\imath}_{n}=\tilde{v}+\tilde{e}_{n}$, where the random variables $\tilde{v}, \tilde{z}, \tilde{e}_{1}, \ldots, \tilde{e}_{N}$ are assumed to be normally and independently distributed with zero means and variances given by $\operatorname{var}[\tilde{v}]=\tau_{v}^{-1}, \operatorname{var}[\tilde{z}]=\sigma_{z}^{2}$, $\operatorname{var}\left[\tilde{e}_{n}\right]=\tau_{e}^{-1} \cdot{ }^{4}$

Speculators compete in demand schedules and have constant absolute risk-aversion utility functions with coefficient $\rho>0$ and a (normalized) zero initial endowment of the risky asset. Speculator $n$ chooses a demand schedule $X_{n}\left(\cdot, i_{n}\right)$ which depends on his signal $i_{n}$ and, given the market clearing price $p$, derives utility

$$
U_{n}\left(\pi_{n}\right)=-\exp \left(-\rho \pi_{n}\right) \quad \text { where } \pi_{n}=(v-p) x_{n} \text { and } x_{n}=X_{n}\left(p, i_{n}\right) .
$$

Market makers are uninformed and make their trade based only on public information transmitted through the price. We assume that there is a competitive risk-neutral market making sector that induces semi-strong efficient pricing:

$$
E[\tilde{v} \mid \tilde{p}]=\tilde{p}
$$

This may arise because uninformed traders are risk neutral or because there is costless free entry of uninformed speculators (as we will see in the next section).

Strategic and competitive equilibria Two equilibria are considered. The first is a competitive REE and the second is Kyle's REE with imperfect competition. The second is simply a Bayesian equilibrium in demand schedules of the game among the $N$ informed traders. The first is also a Bayesian equilibrium, but now each informed trader, when considering what demand schedule to use, does not take into account the impact of his choice on the market price.

We concentrate attention on symmetric linear equilibria, that is, an equilibrium in which the strategies for each trader $X_{n}, n=1, \ldots, N$, are identical affine functions. Thus, there exist constants $\beta, \gamma, \mu$ such that a strategy $X_{n}$ can be written

$$
X_{n}\left(p, i_{n}\right)=\mu+\beta i_{n}-\gamma p .
$$

\footnotetext{
${ }^{3} \mathrm{~A}$ tilde distinguishes between a random variable and its realization.

${ }^{4}$ The assumption $E[\tilde{v}]=E\left[\tilde{e}_{n}\right]=0$ is made without loss of generality. The assumption $E[\tilde{z}]=0$ does not affect the results in this paper.
} 
Theorem 5.1 in Kyle (1989) implies that for $\sigma_{z}^{2}>0$ and $\tau_{e}>0$ there exists a unique symmetric linear REE with imperfect competition for any $N$. That is, there is a symmetric linear equilibrium and this equilibrium is unique in the class of symmetric linear equilibria. Theorem 6.1 in Kyle (1989) provides a corresponding result on the existence and uniqueness of a symmetric linear competitive REE.

We use a superscript $\mathrm{c}$ to denote the values associated with a competitive equilibrium; values associated with a strategic equilibrium do not have this superscript. Subscript $N$ (respectively, subscript $\infty$ ) corresponds to the values of the market with $N$ (respectively, with an infinite number of) informed traders.

\section{$3 \quad$ Free entry in a large market}

Consider the following scenario in which we endogenize the number of informed speculators. There are two stages and a countable infinity of potential traders. In a first stage any trader (except noise traders) can become informed (that is, can receive a signal about the value of the asset) by paying a fixed amount $F>0$. In a second stage the speculators that have decided to enter compete as in the previous section to make money out of the noise traders. Free entry of uninformed speculators, even if they are risk averse, implies that the market at the second stage is semi-strong efficient (Theorem 7.4 in Kyle (1989)). ${ }^{5}$

The size of the market is naturally parameterized by the noise trading volume $\sigma_{z}$. We also index the cost of acquiring information by $\sigma_{z}: F\left(\sigma_{z}\right)$. If $F(\cdot)$ is constant there are constant returns to information acquisition. If $F(\cdot)$ is increasing (decreasing) in the size of the market there are decreasing (increasing) returns to scale to information acquisition. Increasing returns to information production may arise because of fixed costs in information production and decreasing returns may arise because of increased correlation among signals when a larger sample is produced. The central case is with constant returns and $F(\cdot) \equiv F>0$. Assume that

$$
F(\infty) \equiv \lim _{\sigma_{z} \rightarrow \infty} F\left(\sigma_{z}\right)
$$

is well defined.

The equilibrium number of informed traders is determined as follows. Consider first the strategic case. Let $\tilde{\pi}_{N}$ be the equilibrium random profits of an informed speculator when $N$ have entered and denote by $\Pi(N)$ the certainty equivalent of profits that makes a trader indifferent between making this payment to be one of the informed speculators or remain uninformed. We have that, because of exponential utility, $\Pi(N)$ is independent of initial wealth and

$$
-\exp (-\rho \Pi(N))=E\left[-\exp \left(-\rho \tilde{\pi}_{N}\right)\right]
$$

\footnotetext{
${ }^{5}$ Alternatively, we may assume that at the second stage there is a competitive risk-neutral market making sector and then no risk-averse trader will choose to enter if he does not purchase information.
} 
Let $N^{*}\left(\sigma_{z}\right)$ denote the equilibrium number of informed speculators in the imperfect competition model when noise trading has standard deviation $\sigma_{z}$. Here $N^{*}\left(\sigma_{z}\right)$ is defined as the largest $N$ such that

$$
\Pi(N) \geq F\left(\sigma_{z}\right)
$$

Let us define the competitive equilibrium model $\Pi^{\mathrm{c}}(N)$ and $N_{\mathrm{c}}^{*}\left(\sigma_{z}\right)$ similarly. Then the equilibrium number of speculators $N_{\mathrm{c}}^{*}\left(\sigma_{z}\right)$ is defined as the largest $N$ such that

$$
\Pi^{\mathrm{c}}(N) \geq F\left(\sigma_{z}\right)
$$

Then, as is shown in Kyle (1989, Theorems 10.1 and 10.2), there is a unique free-entry equilibrium of the two-stage game in both the strategic and competitive cases.

We are interested in the rate of change of the equilibrium number of entrants in both the competitive and the strategic regime as the market becomes large by increasing $\sqrt{\operatorname{var}[\tilde{z}]}=\sigma_{z}$. For all of the markets $\operatorname{var}[\tilde{v}]=\tau_{v}^{-1}$ and $\operatorname{var}\left[\tilde{e}_{n}\right]=\tau_{e}^{-1}$ are fixed constants.

We say that a market with equilibrium price $\tilde{p}$ is (i) value revealing if $\tilde{p}=\tilde{v}$ (almost surely); (ii) informationally trivial if $\operatorname{var}^{-1}[\tilde{v} \mid \tilde{p}]=\operatorname{var}^{-1}[\tilde{v}]=\tau_{v}$. It is easy to see that in case (ii), necessarily, $\tilde{p}=E[\tilde{v}]=0$ (almost surely). ${ }^{6}$

Notation for the comparison of rates of convergence We use the following notation to make comparisons of the rates of convergence. For two functions $f, g: \mathbf{Z}_{+} \rightarrow \mathbf{R}$ we have:

(i) $f \sim O(g)$ means that there exist an integer $N_{0}$ and a positive constant $k$ such that $|f(N)| \leq k|g(N)|$ for any $N \geq N_{0}$; that is, $|f|$ grows "at a rate not larger" than $|g|$ as $N \rightarrow \infty$;

(ii) $f \sim o(g)$ means that $\lim _{N \rightarrow \infty}(f(N) / g(N))=0$; that is, $|f|$ grows "at a smaller rate" than $|g|$ as $N \rightarrow \infty$;

(iii) $f \propto g$ means that $f \sim O(g)$ and $g \sim O(f)$; that is, $|f|$ grows "at the same rate" as $|g|$ when $N \rightarrow \infty$.

Our first result shows what cases arise depending on the value of $F(\infty)$.

Proposition 1. Let $\rho>0$ and

$$
F^{*} \equiv \frac{1}{2 \rho} \log \left(1+\frac{\tau_{e}}{\tau_{v}}\right) .
$$

\footnotetext{
${ }^{6}$ Indeed, from $E[\tilde{v} \mid \tilde{p}]=\tilde{p}$ we have that $E[\tilde{p}]=E[E[\tilde{v} \mid \tilde{p}]]=E[\tilde{v}]=0$. Since $\operatorname{var}[\tilde{v}]=E[\operatorname{var}[\tilde{v} \mid \tilde{p}]]+$ $\operatorname{var}[E[\tilde{v} \mid \tilde{p}]]$, we have that $E[\operatorname{var}[\tilde{v} \mid \tilde{p}]]=E[\operatorname{var}[\tilde{v}]]=\operatorname{var}[\tilde{v}]$ and $\operatorname{var}[E[\tilde{v} \mid \tilde{p}]]=\operatorname{var}[\tilde{p}]$ and therefore it should be that $\operatorname{var}[\tilde{p}]=0$.
} 
In a large market three cases appear (in both the strategic and competitive cases) in a free entry equilibrium as $\sigma_{z} \rightarrow \infty$.

1. If $F(\infty) \geq F^{*}$, then if the endogenous number of informed speculators grows, it grows at a slower rate than $\sigma_{z}\left(N_{\mathrm{c}}^{*}\left(\sigma_{z}\right)\right.$ and $N^{*}\left(\sigma_{z}\right)$ are $\left.\sim o\left(\sigma_{z}\right)\right)$. The limit market is informationally trivial and the prices converge to zero.

2. If $0<F(\infty)<F^{*}$, then the endogenous number of informed speculators grows proportionally to $\sigma_{z}\left(N_{\mathrm{c}}^{*}\left(\sigma_{z}\right) \propto N^{*}\left(\sigma_{z}\right) \propto \sigma_{z}\right)$. The limit market is neither value revealing nor informationally trivial.

3. If $F(\infty)=0$, then the endogenous number of informed speculators grows at a faster rate than $\sigma_{z}\left(\sigma_{z} \sim o\left(N_{\mathrm{c}}^{*}\left(\sigma_{z}\right)\right)\right.$ and $\sigma_{z} \sim o\left(N^{*}\left(\sigma_{z}\right)\right)$. The limit market is value revealing: the prices converge to $\tilde{v}$.

The limit market in case (2) corresponds to the continuum of traders competitive model of Vives (1995) where informed traders co-exist with risk neutral market makers. The competitive models of Hellwig (1980) and Admati (1985) do not have risk neutral market makers. The result is also consistent with related work by García and Urosevic (2008) (where they also find a limit of the Vives (1995) type corresponding to their case of "diversifiable noise".) Remark 1 . In the case of constant returns to information acquisition, $F(\cdot) \equiv F$ :

1. if $F \geq F^{*}$, then $N_{\mathrm{c}}^{*}\left(\sigma_{z}\right)=N^{*}\left(\sigma_{z}\right)=0$;

2. if $0<F<F^{*}$, then $N_{\mathrm{c}}^{*}\left(\sigma_{z}\right) \propto N^{*}\left(\sigma_{z}\right) \propto \sigma_{z}$;

3. if $F=0$, then $N_{\mathrm{c}}^{*}\left(\sigma_{z}\right)=N^{*}\left(\sigma_{z}\right)=\infty$.

The proofs of all results are collected together in the Appendix. Let us present here a sketch of the argument.

An heuristic explanation of the proof Consider first the strategic case for an exogenous number of speculators $N$. It is easy to see that the certainty equivalent of profits $\Pi(N)$ is strictly positive and is strictly smaller than

$$
F^{*} \equiv \frac{1}{2 \rho} \log \left(1+\frac{\tau_{e}}{\tau_{v}}\right)
$$

Then it is shown that $\Pi(N)$ converges as $\sigma_{z} \rightarrow \infty$ to different limits depending on the rate of the growth of $N$ :

(i) if $N$ grows at a slower rate than $\sigma_{z}$, then relatively few informed speculators are in the market, the limit market is informationally trivial, and the certainty equivalent of profits $\Pi(N)$ converges to its upper bound $F^{*}$ (information is the most valuable); 
(ii) if $N$ grows at the same rate as $\sigma_{z}$, then the limit market is neither value revealing nor informationally trivial, and the certainty equivalent of profits $\Pi(N)$ for large $N$ belongs to some interval strictly between 0 and $F^{*}$ (information is valuable);

(iii) if $N$ grows at a faster rate than $\sigma_{z}$, then relatively many speculators are in the market, the limit market is value revealing and the certainty equivalent of profits $\Pi(N)$ converges to 0 (information is not valuable).

Allow now for an endogenous number of speculators $N$. If $N$ does not grow unboundedly as $\sigma_{z} \rightarrow \infty$, then we should be in the case (i) above and $F(\infty) \geq F^{*}$ (with no speculators becoming informed when $F(\cdot) \equiv F$ ). If $N$ grows unboundedly as $\sigma_{z} \rightarrow \infty$ then it should be that $\Pi(N) \rightarrow F(\infty)$. (For a given $\sigma_{z}$ speculators will enter until $\Pi(N)$ is just above $F(\infty)$.) Depending on the value of $F(\infty)$ we should be in one of the cases (i)-(iii) above. If $F(\infty) \geq F^{*}$, then we must be in case (i) since eventually it does not pay to become informed. If $F(\infty)=0$, then eventually all speculators enter and we must be in case (iii). If $0<F(\infty)<F^{*}$, then $N$ must grow unboundedly with $\sigma_{z}$ and $\Pi(N) \rightarrow F(\infty)$ (otherwise, $\Pi(N) \rightarrow F^{*}$ and there would be more entry since $\left.F(\infty)<F^{*}\right)$. It follows that we must be in case (ii) since if $N$ grows at a faster rate than $\sigma_{z}$, then $\Pi(N) \rightarrow 0$, and if $N$ grows at a slower rate than $\sigma_{z}$, then $\Pi(N) \rightarrow F^{*}$. We can show in this case that $\sigma_{z} / N^{*}\left(\sigma_{z}\right) \rightarrow \sigma_{z_{0}}$ as $\sigma_{z} \rightarrow \infty$ for some constant $\sigma_{z_{0}}$ inversely related to $F(\infty)$.

For the price-taking equilibrium the analysis is similar but based on $\Pi^{\mathrm{c}}(N)$.

Hence, in general, for the whole range of intermediate values of $F(\infty)$ we obtain that the endogenous number of informed speculators is proportional to the standard deviation of the noise trade and this is the case that has as its limit the usual continuum model. Thus, it is natural to consider sequences of markets where the numbers of informed speculators are proportional to $\sigma_{z}$. In the next section we restrict our attention to such sequences of markets. After the presentation of our main results, we present some complementary results for different sequences of markets in the following sections.

\section{Convergence to price-taking in the central scenario}

Let us consider the following sequence of markets indexed by $N$. At the $N$ th market there are $N$ informed agents. In all of the markets $\operatorname{var}[\tilde{v}]=\tau_{v}^{-1}$ and $\operatorname{var}\left[\tilde{e}_{n}\right]=\tau_{e}^{-1}$ are fixed constants. Let $\tilde{z}(N)=N \tilde{z}_{0}$ and $\operatorname{var}\left[\tilde{z}_{0}\right]=\sigma_{z_{0}}^{2}$, where $\sigma_{z_{0}}^{2}$ is a constant. That is, the standard deviation of the noise trade $\sigma_{z}$ grows at a rate of $N$.

As $N$ grows we know that the strategic and competitive equilibria tend to each other (from Theorem 9.2 in Kyle (1989)) and that they both tend to the competitive equilibrium of the limit continuum economy (as in Hellwig (1980), Admati (1985) or Vives (1995)). We now characterize the rate at which strategic and competitive equilibria tend to each other and the rate at which they tend to the competitive equilibrium of the limit economy. 
Convergence concepts To compare rates of convergence of random variables we use the square loss function. We say that two random variables $x, y$ converge to each other at some rate if $\sqrt{E\left[(x-y)^{2}\right]}$ converges to zero at this rate. Note that

$$
E\left[(x-y)^{2}\right]=(E[x]-E[y])^{2}+\operatorname{var}[x-y],
$$

and that if $E[x]=E[y]$, then $E\left[(x-y)^{2}\right]=\operatorname{var}[x-y]$.

A more refined measure of convergence speed for a given convergence rate is provided by the asymptotic standard deviation. Suppose that $\sqrt{E\left[\left(x_{N}-y_{N}\right)^{2}\right]}=\sqrt{\operatorname{var}\left[x_{N}-y_{N}\right]}$ converges to zero at a rate of $1 / N^{\alpha}$ for some $\alpha>0$ (that is, $\sqrt{\operatorname{var}\left[x_{N}-y_{N}\right]} \propto 1 / N^{\alpha}$ ), then the asymptotic standard deviation of convergence is given by the constant $\lim _{N \rightarrow \infty} N^{\alpha} \sqrt{\operatorname{var}\left[x_{N}-y_{N}\right]}$. A higher asymptotic standard deviation means that the speed of convergence is slower.

\section{$4.1 \quad$ Prices}

In order to compare convergence rates for prices consider the following decomposition:

$$
\tilde{p}_{N}-\tilde{p}_{\infty}=\left(\tilde{p}_{N}-\tilde{p}_{N}^{\mathrm{c}}\right)+\left(\tilde{p}_{N}^{\mathrm{c}}-\tilde{p}_{\infty}\right)
$$

The first term of the decomposition captures the difference between equilibrium prices for the price-taking $\tilde{p}_{N}^{\mathrm{c}}$ and strategic equilibria $\tilde{p}_{N}$ in the same finite market. The second term captures the change in the competitive price from the finite to the limit market. We have that

$$
E\left[\left(\tilde{p}_{N}-\tilde{p}_{\infty}\right)^{2}\right]=E\left[\left(\tilde{p}_{N}-\tilde{p}_{N}^{\mathrm{c}}\right)^{2}\right]+E\left[\left(\tilde{p}_{N}^{\mathrm{c}}-\tilde{p}_{\infty}\right)^{2}\right]+2 \operatorname{Cov}\left[\tilde{p}_{N}-\tilde{p}_{N}^{\mathrm{c}}, \tilde{p}_{N}^{\mathrm{c}}-\tilde{p}_{\infty}\right]
$$

will be of the order of the higher order term. Using Hölder's inequality (see, e.g., Royden (1968, p. 113)) we obtain that

$$
\operatorname{Cov}\left[\tilde{p}_{N}-\tilde{p}_{N}^{\mathrm{c}}, \tilde{p}_{N}^{\mathrm{c}}-\tilde{p}_{\infty}\right] \leq\left(E\left[\left(\tilde{p}_{N}-\tilde{p}_{N}^{\mathrm{c}}\right)^{2}\right]\right)^{1 / 2}\left(E\left[\left(\tilde{p}_{N}^{\mathrm{c}}-\tilde{p}_{\infty}\right)^{2}\right]\right)^{1 / 2}
$$

and therefore the interaction covariance term will be of lower order than the higher-order term of $E\left[\left(\tilde{p}_{N}-\tilde{p}_{N}^{\mathrm{c}}\right)^{2}\right]$ or $E\left[\left(\tilde{p}_{N}^{\mathrm{c}}-\tilde{p}_{\infty}\right)^{2}\right]$. The term $E\left[\left(\tilde{p}_{N}-\tilde{p}_{N}^{\mathrm{c}}\right)^{2}\right]$ corresponds to the strategic effect and the term $E\left[\left(\tilde{p}_{N}^{\mathrm{c}}-\tilde{p}_{\infty}\right)^{2}\right]$ corresponds to the limit effect.

We show in the next result that $E\left[\left(\tilde{p}_{N}-\tilde{p}_{N}^{\mathrm{c}}\right)^{2}\right]$ converges to zero faster than $E\left[\left(\tilde{p}_{N}^{\mathrm{c}}-\tilde{p}_{\infty}\right)^{2}\right]$ and therefore $E\left[\left(\tilde{p}_{N}-\tilde{p}_{\infty}\right)^{2}\right]$ inherits the order of $E\left[\left(\tilde{p}_{N}^{\mathrm{c}}-\tilde{p}_{\infty}\right)^{2}\right]$ (they both converge to zero at exactly the same speed).

Given competitive market making, the expectations of the differences in equilibrium prices vanish and we need to compare only the rates of convergence of variances.

Proposition 2. For a sequence of markets described above: 
1. $\sqrt{E\left[\left(\tilde{p}_{N}-\tilde{p}_{N}^{\mathrm{c}}\right)^{2}\right]}=\sqrt{\operatorname{var}\left[\tilde{p}_{N}-\tilde{p}_{N}^{\mathrm{c}}\right]} \propto 1 / N$

2. $\sqrt{E\left[\left(\tilde{p}_{N}^{\mathrm{c}}-\tilde{p}_{\infty}\right)^{2}\right]}=\sqrt{\operatorname{var}\left[\tilde{p}_{N}^{\mathrm{c}}-\tilde{p}_{\infty}\right]} \propto 1 / \sqrt{N} ;$ and, therefore,

3. $\sqrt{E\left[\left(\tilde{p}_{N}-\tilde{p}_{\infty}\right)^{2}\right]}=\sqrt{\operatorname{var}\left[\tilde{p}_{N}-\tilde{p}_{\infty}\right]} \propto 1 / \sqrt{N}$.

The formal proof is given in the Appendix. Let us present here an informal explanation of the result.

An heuristic explanation The demand of an informed trader at a strategic equilibrium is given by

$$
X_{n}\left(\tilde{p}, \tilde{\imath}_{n}\right)=\frac{E\left[\tilde{v} \mid \tilde{p}, \tilde{\imath}_{n}\right]-\tilde{p}}{\rho \operatorname{var}\left[\tilde{v} \mid \tilde{p}, \tilde{\imath}_{n}\right]+\lambda_{\mathrm{I}}}
$$

where $\lambda_{\mathrm{I}}$ is the slope of inverse supply facing the individual informed trader. In the competitive case $\lambda_{\mathrm{I}}=0$. It is easy to $\operatorname{check}^{7}$ that $\lambda_{\mathrm{I}}$ is of the order of $1 / N$ and this explains why market power vanishes at the rate $1 / N$. (Result 1 in Proposition 2.)

In a symmetric linear equilibrium, prices have the following form:

$$
\begin{gathered}
\tilde{p}_{N}^{\mathrm{c}}=A_{N}^{\mathrm{c}} \tilde{v}+B_{N}^{\mathrm{c}} \frac{1}{N} \sum_{n=1}^{N} \tilde{e}_{n}+C_{N}^{\mathrm{c}} \tilde{z}_{0}, \\
\tilde{p}_{N}=A_{N} \tilde{v}+B_{N} \frac{1}{N} \sum_{n=1}^{N} \tilde{e}_{n}+C_{N} \tilde{z}_{0}, \\
\tilde{p}_{\infty}=A_{\infty} \tilde{v}+C_{\infty} \tilde{z}_{0} .
\end{gathered}
$$

We have that $A_{N}^{\mathrm{c}}$ and $A_{N}$ converge to $A_{\infty}, B_{N}^{\mathrm{c}}$ and $B_{N}$ converge to $B_{\infty} \neq 0$ and $C_{N}^{\mathrm{c}}$ and $C_{N}$ converge to $C_{\infty}$, all at a rate of $1 / N$ or faster. Since we have assumed that $E[\tilde{v}]=E\left[\tilde{e}_{n}\right]=$ $E[\tilde{z}]=0$ expectations of prices are simply zeros (in fact, the expectations of price differences would be zero even if $E[\tilde{v}]>0$ because $E\left[\tilde{p}_{N}^{\mathrm{c}}\right]=E\left[\tilde{p}_{N}\right]=E[\tilde{v}]$ from $p=E[v \mid p]$ in both cases). Therefore, only variances of the price differences are important:

$$
\begin{gathered}
\operatorname{var}\left[\tilde{p}_{N}-\tilde{p}_{N}^{\mathrm{c}}\right]=\left(A_{N}-A_{N}^{\mathrm{c}}\right)^{2} \tau_{v}^{-1}+\left(B_{N}-B_{N}^{\mathrm{c}}\right)^{2} \frac{1}{N} \tau_{e}^{-1}+\left(C_{N}-C_{N}^{\mathrm{c}}\right)^{2} \sigma_{z_{0}}^{2} \sim O\left(1 / N^{2}\right), \\
\operatorname{var}\left[\tilde{p}_{N}^{\mathrm{c}}-\tilde{p}_{\infty}\right]=\left(A_{N}^{\mathrm{c}}-A_{\infty}\right)^{2} \tau_{v}^{-1}+\left(B_{N}^{\mathrm{c}}\right)^{2} \frac{1}{N} \tau_{e}^{-1}+\left(C_{N}^{\mathrm{c}}-C_{\infty}\right)^{2} \sigma_{z_{0}}^{2} \propto 1 / N, \\
\operatorname{var}\left[\tilde{p}_{N}-\tilde{p}_{\infty}\right]=\left(A_{N}-A_{\infty}\right)^{2} \tau_{v}^{-1}+\left(B_{N}\right)^{2} \frac{1}{N} \tau_{e}^{-1}+\left(C_{N}-C_{\infty}\right)^{2} \sigma_{z_{0}}^{2} \propto 1 / N .
\end{gathered}
$$

Thus, $\tilde{p}_{N}^{\mathrm{c}}$ and $\tilde{p}_{N}$ converge to each other faster than to the limit price $\tilde{p}_{\infty}$ (at the rate at which market power vanishes) because prices in the finite markets depend in a similar way on the

\footnotetext{
${ }^{7}$ Conditions (B.8) and (B.9) in Kyle (1989) imply that $\lambda_{\mathrm{I}}=\xi_{\mathrm{I}} /\left(\left(1-\xi_{\mathrm{I}}\right) \gamma\right)$ where the parameter $\xi_{\mathrm{I}}$ can be interpreted as the marginal market share of an informed trader. We have then that $\lambda_{\mathrm{I}} \propto \xi_{\mathrm{I}} \propto 1 / N$ (see Lemma 3 in the Appendix).
} 
average noise in private information $(1 / N) \sum_{n=1}^{N} \tilde{e}_{n}$, while in the continuum limit market the average noise of information cancels out according to the Strong Law of Large Numbers. The distance from $\tilde{p}_{N}^{\mathrm{c}}\left(\right.$ or $\left.\tilde{p}_{N}\right)$ to the limit price $\tilde{p}_{\infty}$ depends on $(1 / N) \sum_{n=1}^{N} \tilde{e}_{n}$ and this average error term converges to zero at a rate of $1 / \sqrt{N}$. (Results 2 and 3 in Proposition 2.) It is worth to remark that the limit effect would disappear if all informed traders were to receive the same signal (indeed, then $\tilde{p}_{N}^{\mathrm{c}}=\tilde{p}_{\infty}$ ).

Asymptotic variances of convergence Note also that in Proposition 2 we actually show slightly more than in the above heuristic argument. Namely, we prove that $\tilde{p}_{N}$ and $\tilde{p}_{N}^{\mathrm{c}}$ converge to each other precisely at the rate $1 / N$. We can also characterize the asymptotic variances of convergence. Those gives us a refined measure of the speed of convergence for a given convergence rate.

Proposition 3. Let $A_{\mathrm{L}}$ denote the asymptotic standard deviation of the limit effect and $A_{\mathrm{S}}$ denote the one of the strategic effect. Then:

1. $\lim _{N \rightarrow \infty} N \sqrt{\operatorname{var}\left[\tilde{p}_{N}-\tilde{p}_{N}^{\mathrm{c}}\right]}=A_{\mathrm{S}} \equiv \frac{\tau_{e}^{2}}{\sigma_{z_{0}} \rho\left(\tau_{e}^{2}+\tau_{v} \sigma_{z_{0}}^{2} \rho^{2}\right)}\left(1+\frac{\tau_{e} \sigma_{z_{0}}^{2} \rho^{2}}{\tau_{e}^{2}+\tau_{v} \sigma_{z_{0}}^{2} \rho^{2}}\right)$
2. $\lim _{N \rightarrow \infty} \sqrt{N} \sqrt{\operatorname{var}\left[\tilde{p}_{N}^{\mathrm{c}}-\tilde{p}_{\infty}\right]}=\lim _{N \rightarrow \infty} \sqrt{N} \sqrt{\operatorname{var}\left[\tilde{p}_{N}-\tilde{p}_{\infty}\right]}=A_{\mathrm{L}} \equiv \frac{\tau_{e}^{3 / 2}}{\tau_{e}^{2}+\tau_{v} \sigma_{z_{0}}^{2} \rho^{2}}$.

\subsubsection{Comparative statics}

Comparative statics for the limit effect We have that

$$
A_{\mathrm{L}}=\frac{\tau_{e}^{3 / 2}}{\tau_{e}^{2}+\tau_{v} \sigma_{z_{0}}^{2} \rho^{2}}
$$

The following comparative statics results are immediate.

(1) $A_{\mathrm{L}}$ monotonically decreases in the precision of the prior $\tau_{v}$. If $\tau_{v} \rightarrow 0+$, then $A_{\mathrm{L}} \rightarrow$ $1 / \sqrt{\tau_{e}}$. If $\tau_{v} \rightarrow+\infty$, then $A_{\mathrm{L}} \rightarrow 0$. This result is quite intuitive. The lower the variance of the value of the asset, the faster equilibrium prices converge to the limit.

(2) $A_{\mathrm{L}}$ monotonically decreases in "risk-bearing adjusted noise trade" $\rho \sigma_{z_{0}}$. If $\rho \sigma_{z_{0}} \rightarrow 0+$, then $A_{\mathrm{L}} \rightarrow 1 / \sqrt{\tau_{e}}$. If $\rho \sigma_{z_{0}} \rightarrow+\infty$, then $A_{\mathrm{L}} \rightarrow 0$. The fact that $A_{\mathrm{L}}$ decreases in $\rho \sigma_{z_{0}}$ may seem surprising. How can it be that more noise trading or a higher degree of risk aversion improve the convergence to the limit price? However, recall that a term corresponding to the average information noise in the finite market $\left((1 / N) \sum_{n=1}^{N} \tilde{e}_{n}\right)$ determines the convergence to the limit price. The more traders are risk averse the less weight they put on their signals and the less information noise is incorporated into 
the equilibrium price. Similarly, the larger noise trading is the smaller the information noise portion incorporated into the equilibrium price. Thus, an increase in $\rho \sigma_{z_{0}}$ causes a decrease in the amount of information noise incorporated into the price and therefore decreases the asymptotic variance of the limit effect.

(3) $A_{\mathrm{L}}$ is always nonmonotonic in the precision of the signal $\tau_{e}$. If $\tau_{e} \rightarrow 0+$ or if $\tau_{e} \rightarrow$ $+\infty$, then $A_{\mathrm{L}} \rightarrow 0$. Actually, $A_{\mathrm{L}}$ is increasing for $\tau_{e}<\sigma_{z_{0}} \rho \sqrt{3 \tau_{v}}$ and decreasing for $\tau_{e}>\sigma_{z_{0}} \rho \sqrt{3 \tau_{v}}$. At $\tau_{e}=\sigma_{z_{0}} \rho \sqrt{3 \tau_{v}}, A_{\mathrm{L}}$ reaches a maximum. Thus, if signals become very informative or very noisy, then $A_{\mathrm{L}}$ becomes arbitrary small and equilibrium prices converge to the limit faster. Indeed, in both cases the average noise in the signals does not matter much in determining the price in a finite market: when signals are very noisy traders put very little weight on them, and when they are very precise signal noise is very small.

Comparative statics for the strategic effect We have that

$$
A_{\mathrm{S}}=\frac{\tau_{e}^{2}}{\sigma_{z_{0}} \rho\left(\tau_{e}^{2}+\tau_{v} \sigma_{z_{0}}^{2} \rho^{2}\right)}\left(1+\frac{\tau_{e} \sigma_{z_{0}}^{2} \rho^{2}}{\tau_{e}^{2}+\tau_{v} \sigma_{z_{0}}^{2} \rho^{2}}\right)=A_{\mathrm{L}} \frac{\tau_{e}^{1 / 2}}{\sigma_{z_{0}} \rho}\left(1+\frac{1}{\tau_{e} / \sigma_{z_{0}}^{2} \rho^{2}+\tau_{v} / \tau_{e}}\right) .
$$

The following comparative statics results are immediate.

(1) $A_{\mathrm{S}}$ monotonically decreases in $\tau_{v}$. If $\tau_{v} \rightarrow 0+$ then $A_{\mathrm{S}} \rightarrow\left(\sigma_{z_{0}} \rho\right)^{-1}+\tau_{e}^{-1}\left(\sigma_{z_{0}} \rho\right)$. If $\tau_{v} \rightarrow+\infty$ then $A_{\mathrm{S}} \rightarrow 0$. Again, the lower the variance of the value of the asset is, the faster the strategic effect disappears.

(2) $A_{\mathrm{S}}$ either monotonically decreases or is nonmonotonic in $\rho \sigma_{z_{0}}$ depending on other parameters. (For example, if $\tau_{e}=1$ and $\tau_{v}=1$, then $A_{\mathrm{S}}$ monotonically decreases in $\rho \sigma_{z_{0}}$. However, if $\tau_{e}=1$ and $\tau_{v}=0.01$, then for $\rho \sigma_{z_{0}}=0.5$ we obtain $A_{\mathrm{S}}=2.49$; for $\rho \sigma_{z_{0}}=1$ we obtain $A_{\mathrm{S}}=1.97$; and for $\rho \sigma_{z_{0}}=2$ we obtain $A_{\mathrm{S}}=2.33$.) Furthermore, if $\rho \sigma_{z_{0}} \rightarrow 0+$, then $A_{\mathrm{S}} \rightarrow+\infty$. If $\rho \sigma_{z_{0}} \rightarrow+\infty$, then $A_{\mathrm{S}} \rightarrow 0$. If the noise trading is small for the risk-bearing capacity of the informed traders, then the strategic effect disappears more slowly. In fact, with risk neutrality competitive prices become fully revealing and the strategic and competitive equilibria converge to each other at a slower rate than in Proposition 2 (since $A_{\mathrm{S}} \rightarrow+\infty$ ). In fact, $\left\{\lim _{\rho \rightarrow 0} \sqrt{\operatorname{var}\left[\tilde{p}_{N}-\tilde{p}_{N}^{\mathrm{c}}\right]}\right\} \propto 1 / \sqrt{N}$ as $\tilde{p}_{N}^{\mathrm{c}}$ $\rightarrow \tilde{v}$ for $\rho \rightarrow 0$. If the noise trade is large for the risk-bearing capacity of the informed traders the opposite happens $\left(A_{\mathrm{S}} \rightarrow 0\right)$.

(3) $A_{\mathrm{S}}$ is always nonmonotonic in $\tau_{e}$. In general, the behavior of $A_{\mathrm{S}}$ is very complicated. 
However, $A_{\mathrm{S}}$ can be presented as the product of two functions: $A_{\mathrm{S}}=Q R$, where

$$
\begin{aligned}
& Q=\frac{\tau_{e}^{2}}{\sigma_{z_{0}} \rho\left(\tau_{e}^{2}+\tau_{v} \sigma_{z_{0}}^{2} \rho^{2}\right)}=\frac{1}{\sigma_{z_{0}} \rho\left(1+\tau_{v} \sigma_{z_{0}}^{2} \rho^{2} / \tau_{e}^{2}\right)} \quad \text { and } \\
& R=\left(1+\frac{\tau_{e} \sigma_{z_{0}}^{2} \rho^{2}}{\tau_{e}^{2}+\tau_{v} \sigma_{z_{0}}^{2} \rho^{2}}\right)=\left(1+\frac{1}{\tau_{e} / \sigma_{z_{0}}^{2} \rho^{2}+\tau_{v} / \tau_{e}}\right) .
\end{aligned}
$$

Then $Q$ is monotonically increasing in $\tau_{e}$. However, $R$ is increasing in $\tau_{e}$ for $\tau_{e}<$ $\sigma_{z_{0}} \rho \sqrt{\tau_{v}}$ and $R$ is decreasing for $\tau_{e}>\sigma_{z_{0}} \rho \sqrt{\tau_{v}}$. If $\tau_{e} \rightarrow 0+$, then $Q \rightarrow 0$ and $R \rightarrow 1$, so $A_{\mathrm{S}} \rightarrow 0$. Clearly, $A_{\mathrm{S}}$ is increasing in $\tau_{e}$ for $\tau_{e}<\sigma_{z_{0}} \rho \sqrt{\tau_{v}}$. If $\tau_{e} \rightarrow+\infty$, then $Q$ increases to the value of $\left(\sigma_{z_{0}} \rho\right)^{-1}$ in the limit (and it reaches the limiting value at the rate $\left.1 / \tau_{e}^{2}\right)$. However, if $\tau_{e} \rightarrow+\infty$, then $R$ is decreasing to the value of 1 in the limit. Function $R$ reaches the limiting value at the rate $1 / \tau_{e}$. Therefore if $\tau_{e} \rightarrow+\infty$, then $A_{\mathrm{S}} \rightarrow\left(\sigma_{z_{0}} \rho\right)^{-1}$ and $A_{\mathrm{S}}$ is decreasing in $\tau_{e}$. (Alternatively, one can check directly that the sign of the derivative of $A_{\mathrm{S}}$ is negative for $\tau_{e} \rightarrow+\infty$.)

At $\tau_{e}=\sigma_{z_{0}} \rho \sqrt{\tau_{v}}, R$ reaches a maximum of $\left(1+\sigma_{z_{0}} \rho / \sqrt{\tau_{v}}\right)$ while $Q=1 /\left(2 \sigma_{z_{0}} \rho\right)$. Generally $Q<1 / \sigma_{z_{0}} \rho$. Therefore, the maximum value of $A_{\mathrm{S}}$ is between

$$
\frac{1}{2 \sigma_{z_{0}} \rho}\left(1+\frac{\sigma_{z_{0}} \rho}{\sqrt{\tau_{v}}}\right) \text { and } \frac{1}{\sigma_{z_{0}} \rho}\left(1+\frac{\sigma_{z_{0}} \rho}{\sqrt{\tau_{v}}}\right)
$$

Thus, if signals are very noisy, then $A_{\mathrm{S}}$ (as $A_{\mathrm{L}}$ ) becomes arbitrary small. For signals that are very informative $A_{\mathrm{S}}$ reaches some positive number (while $A_{\mathrm{L}}$ becomes arbitrary small). The difference in the behavior of the two asymptotic variances is again a result of the fact that it is not only the information noise that is a factor for the strategic effect.

We have that $A_{\mathrm{S}}>A_{\mathrm{L}} \frac{\tau_{e}^{1 / 2}}{\sigma_{z_{0}} \rho}$. For practical purposes the limit effect will dominate the strategic one whenever $A_{\mathrm{L}} / \sqrt{N}>A_{\mathrm{S}} / N$. Therefore for such domination to work we need to have

$$
\sqrt{N}>\frac{\tau_{e}^{1 / 2}}{\sigma_{z_{0}} \rho} \quad \text { or } \quad N>\frac{\tau_{e}}{\sigma_{z_{0}}^{2} \rho^{2}} .
$$

In summary, we have that $A_{\mathrm{S}}$ is small, and the approximation of the strategic equilibrium by the competitive equilibrium good, when the prior volatility of the asset is low, noise trading is large in relation to the risk-bearing capacity of the informed traders, or the signals are very noisy. We confirm, therefore, the idea that the competitive approximation works even in a moderately sized market when the informationally adjusted risk-bearing capacity of the informed traders is not very large (i.e. basically when competitive traders have incentives to be restrained in their trading). 


\subsubsection{Values for stock markets}

Let us compute the asymptotic variances for some reasonable values of the parameters in a stock market. Let $\rho=2$ and $\sqrt{\operatorname{var}\left[\tilde{z}_{0}\right]}=\sigma_{z_{0}}=0.1$. For the volatility of the fundamentals let us consider two cases: (i) $\sqrt{\operatorname{var}[\tilde{v}]}=\tau_{v}^{-1 / 2}=0.2$ (NYSE type) or (ii) $\sqrt{\operatorname{var}[\tilde{v}]}=\tau_{v}^{-1 / 2}=0.6$ (Nasdaq type). These parameters are chosen to reflect average market data. (See Table 1 in Leland (1992).)

Table 1 presents results for $\sqrt{\operatorname{var}\left[\tilde{e}_{n}\right]}=\tau_{e}^{-1 / 2}$ ranging from 0.05 to 20 . It is immediate from this table that both $A_{\mathrm{S}}$ and $A_{\mathrm{L}}$ are nonmonotonic in $\tau_{e}$. Table 1 is consistent with the statements that $A_{\mathrm{S}}$ and $A_{\mathrm{L}}$ are decreasing in $\tau_{v}$ and that $A_{\mathrm{S}}$ and $A_{\mathrm{L}}$ converge to zero as $\tau_{e} \rightarrow$ 0 . For large $\tau_{e}$, as predicted, $A_{\mathrm{L}}$ converges to zero, while $A_{\mathrm{S}}$ converges to $1 /\left(\rho \sigma_{z_{0}}\right)=5$. The standard deviation of the distance between the strategic and competitive price $\sqrt{\operatorname{var}\left[\tilde{p}_{N}-\tilde{p}_{N}^{\mathrm{c}}\right]}$ is approximated by $A_{\mathrm{S}} / N$. We see that in all scenarios this standard deviation is quite small even with very few informed traders.

\begin{tabular}{|c|c|ccccccc|}
\hline & & \multicolumn{7}{|c|}{$\tau_{e}^{-1 / 2}$} \\
\hline$\tau_{v}^{-1 / 2}$ & & 0.05 & 0.1 & 0.5 & 1 & 5 & 10 & 20 \\
\hline 0.2 & $A_{\mathrm{S}}$ & 5.0005 & 5.0015 & 4.7502 & 2.5500 & 0.0080 & 0.0005 & 0.0000 \\
& $A_{\mathrm{L}}$ & 0.0500 & 0.1000 & 0.4706 & 0.5000 & 0.0080 & 0.0009 & 0.0001 \\
0.6 & $A_{\mathrm{S}}$ & 5.0005 & 5.0019 & 5.0148 & 4.6620 & 0.0720 & 0.0045 & 0.0003 \\
& $A_{\mathrm{L}}$ & 0.0500 & 0.1000 & 0.4966 & 0.9000 & 0.0710 & 0.0090 & 0.0011 \\
\hline
\end{tabular}

Table 1: Values for $A_{\mathrm{S}}$ and $A_{\mathrm{L}}$.

Values for S\&P 500 Futures market In a recent paper Cho and Krishnan (2000) addressed the S\&P 500 Futures market. They found that a competitive rational expectations model provides a reasonable description of this market and present estimates of the primitive parameters of the model (in Table 2 of their paper): the standard deviation of the fundamentals at $\sqrt{\operatorname{var}[\tilde{v}]}=\tau_{v}^{-1 / 2}=5.495$; and for other parameters $\left(\sigma_{z_{0}} \rho\right.$ and $\left.\sqrt{\operatorname{var}\left[\tilde{e}_{n}\right]}=\tau_{e}^{-1 / 2}\right)$ they presented results, which are summarized in Table 2 together with the corresponding values of the asymptotic standard deviations $A_{\mathrm{S}}$ and $A_{\mathrm{L}}$, that differ in the number of the weeks to maturity (from 2 to 7 ).

\begin{tabular}{|c|cccccc|}
\hline & \multicolumn{6}{|c|}{ Time to maturity (weeks) } \\
\hline & 2 & 3 & 4 & 5 & 6 & 7 \\
\hline$\sigma_{z_{0}} \rho$ & 0.021 & 0.007 & 0.045 & 0.038 & 0.030 & 0.029 \\
$\tau_{e}^{-1 / 2}$ & 12.541 & 23.358 & 10.067 & 12.925 & 17.263 & 20.705 \\
$A_{\mathrm{S}}$ & 36.76 & 98.06 & 14.76 & 12.44 & 9.81 & 5.97 \\
$A_{\mathrm{L}}$ & 9.21 & 15.75 & 5.96 & 5.54 & 4.73 & 3.38 \\
\hline
\end{tabular}

Table 2: Summary of results (Cho and Krishnan (2000)). 
The S\&P 500 Futures market has high volatility, very noisy information signals and relatively small noise trade. In this context the asymptotic variances are larger than with the stock market parameters. However, since the number of traders in the S\&P 500 Futures market is very large, the competitive REE should be a very close approximation of the strategic REE in this market. In the analysis of S\&P 500 Futures market one can safely take the shortcut of assuming competitive behavior.

\subsection{Demands, profits and utilities}

We now study the convergence rates for demands, profits and utilities. For all of these we confirm the result that the strategic effect is of the order of $1 / N$ while the limit effect is of the order of $1 / \sqrt{N}$. The first of these results addresses demands.

Proposition 4. We have:

1. $\sqrt{E\left[\left(\tilde{x}_{N}-\tilde{x}_{N}^{\mathrm{c}}\right)^{2}\right]}=\sqrt{\operatorname{var}\left[\tilde{x}_{N}-\tilde{x}_{N}^{\mathrm{c}}\right]} \propto 1 / N$;

2. $\sqrt{E\left[\left(\tilde{x}_{N}^{\mathrm{c}}-\tilde{x}_{\infty}\right)^{2}\right]}=\sqrt{\operatorname{var}\left[\tilde{x}_{N}^{\mathrm{c}}-\tilde{x}_{\infty}\right]} \propto 1 / \sqrt{N}$;

3. $\sqrt{E\left[\left(\tilde{x}_{N}-\tilde{x}_{\infty}\right)^{2}\right]}=\sqrt{\operatorname{var}\left[\tilde{x}_{N}-\tilde{x}_{\infty}\right]} \propto 1 / \sqrt{N}$.

As for prices, the expectations of all demands are zero, so these square loss functions simply are the variances of the differences between corresponding demands. Since demands are linear functions of prices, the same effect holding for prices should hold in terms of demands.

Our next proposition demonstrates a similar conclusion for the profits of the traders.

Proposition 5. We have:

1. $\sqrt{E\left[\left(\tilde{\pi}_{N}-\tilde{\pi}_{N}^{\mathrm{c}}\right)^{2}\right]} \propto 1 / N$;

2. $\sqrt{E\left[\left(\tilde{\pi}_{N}^{\mathrm{c}}-\tilde{\pi}_{\infty}\right)^{2}\right]} \propto 1 / \sqrt{N}$;

3. $\sqrt{E\left[\left(\tilde{\pi}_{N}-\tilde{\pi}_{\infty}\right)^{2}\right]} \propto 1 / \sqrt{N}$.

For profits, unlike for prices or demands, expectations of differences do not vanish, so we cannot restrict attention only to the variances but rather we have to consider the entire square loss functions.

Finally, we consider relative changes in utilities.

Proposition 6. We have: 
1. $\sqrt{E\left[\left(U\left(\tilde{\pi}_{N}^{\mathrm{c}}\right) / U\left(\tilde{\pi}_{N}\right)-1\right)^{2}\right]} \propto 1 / N$

2. $\sqrt{E\left[\left(U\left(\tilde{\pi}_{N}^{\mathrm{c}}\right) / U\left(\tilde{\pi}_{\infty}\right)-1\right)^{2}\right]} \propto 1 / \sqrt{N}$;

3. $\sqrt{E\left[\left(U\left(\tilde{\pi}_{N}\right) / U\left(\tilde{\pi}_{\infty}\right)-1\right)^{2}\right]} \propto 1 / \sqrt{N}$.

Similarly to profits, utilities have non-zero expectations.

\section{Convergence in other scenarios}

In this section we consider how our results are affected if the number of informed speculators does not change proportionally to noise trading. As we have seen above, two different limit cases can emerge. We consider them separately.

\subsection{Fully revealing limit}

We have seen that if the number of informed speculators grows at a faster rate than noise trading, a value revealing limit is obtained. Let us study the convergence rates in two cases: First, when $\sigma_{z}$ grows slower than a rate $N$ but faster than a rate $\sqrt{N}$; second, when $\sigma_{z}$ grows at a rate $\sqrt{N}$ or slower. The following propositions provide the results.

Proposition 7. For a sequence of markets with $\sigma_{z}$ growing slower than at the rate $N$ but faster than at the rate $\sqrt{N}$ we obtain:

1. $\sqrt{\operatorname{var}\left[\tilde{p}_{N}-\tilde{p}_{N}^{\mathrm{c}}\right]} \propto 1 / \sigma_{z}(N) ;$

2. $\sqrt{\operatorname{var}\left[\tilde{p}_{N}^{\mathrm{c}}-\tilde{v}\right]} \propto \sigma_{z}(N) / N$;

3. $\sqrt{\operatorname{var}\left[\tilde{p}_{N}-\tilde{v}\right]} \propto \sigma_{z}(N) / N$.

Therefore, since $1 / \sigma_{z}(N)=\left(\sigma_{z}(N) / N\right)\left(N / \sigma_{z}^{2}(N)\right) \sim o\left(\sigma_{z}(N) / N\right)$, the strategic effect vanishes faster than the limit effect as in our central scenario.

The reader may notice that if $\sigma_{z}(N)$ grows almost at a rate $\sqrt{N}$, then in Proposition 7 the strategic effect is almost of the same order as the limit effect. The next proposition shows that if $\sigma_{z}(N)$ grows at a rate $\sqrt{N}$, then the rate of convergence is $1 / \sqrt{N}$, but the strategic effect is still dominated by the limit effect if one considers the asymptotic variances.

Proposition 8. For a sequence of markets where $\sigma_{z}$ grows at the rate $\sqrt{N}$, we obtain:

1. $\sqrt{\operatorname{var}\left[\tilde{p}_{N}-\tilde{p}_{N}^{\mathrm{c}}\right]} \propto \sqrt{\operatorname{var}\left[\tilde{p}_{N}^{\mathrm{c}}-\tilde{v}\right]} \propto \sqrt{\operatorname{var}\left[\tilde{p}_{N}-\tilde{v}\right]} \propto 1 / \sqrt{N} ;$ 
2. $\lim _{N \rightarrow \infty} \sqrt{N} \sqrt{\operatorname{var}\left[\tilde{p}_{N}-\tilde{p}_{N}^{\mathrm{c}}\right]}<\lim _{N \rightarrow \infty} \sqrt{N} \sqrt{\operatorname{var}\left[\tilde{p}_{N}-\tilde{v}\right]} ;$

3. $\lim _{N \rightarrow \infty} \sqrt{N} \sqrt{\operatorname{var}\left[\tilde{p}_{N}^{\mathrm{c}}-\tilde{v}\right]}<\lim _{N \rightarrow \infty} \sqrt{N} \sqrt{\operatorname{var}\left[\tilde{p}_{N}-\tilde{v}\right]}$.

Part 2 of Proposition 8 shows that, in terms of asymptotic variances, $\tilde{p}_{N}^{\mathrm{c}}$ converges to $\tilde{p}_{N}$ faster than $\tilde{p}_{N}$ converges to $\tilde{v}$. So $\tilde{p}_{N}^{\mathrm{c}}$ is a better proxy than $\tilde{v}$ for $\tilde{p}_{N}$. Moreover, Part 3 of Proposition 8 demonstrates that in terms of asymptotic variances $\tilde{p}_{N}^{\mathrm{c}}$ converges to $\tilde{v}$ faster than $\tilde{p}_{N}$ converges to $\tilde{v}$ We could say that $\tilde{p}_{N}^{\mathrm{c}}$ is "in between" $\tilde{p}_{N}$ and $\tilde{v}$.

Finally, for sequence of markets in which $\sigma_{z}$ grows slower than at the rate $\sqrt{N}$, a similar result to the previous proposition holds. The main difference is that in this case we have simple expressions for the asymptotic standard deviations.

Proposition 9. For a sequence of markets where $\sigma_{z}$ grows at the rate slower than $\sqrt{N}$ we obtain:

1. $\sqrt{\operatorname{var}\left[\tilde{p}_{N}-\tilde{p}_{N}^{\mathrm{c}}\right]} \propto 1 / \sqrt{N}$ and $\lim _{N \rightarrow \infty} \sqrt{N} \sqrt{\operatorname{var}\left[\tilde{p}_{N}-\tilde{p}_{N}^{\mathrm{c}}\right]}=\sqrt{\tau_{e}^{-1}}$;

2. $\sqrt{\operatorname{var}\left[\tilde{p}_{N}^{\mathrm{c}}-\tilde{v}\right]} \propto 1 / \sqrt{N}$ and $\lim _{N \rightarrow \infty} \sqrt{N} \sqrt{\operatorname{var}\left[\tilde{p}_{N}^{\mathrm{c}}-\tilde{v}\right]}=\sqrt{\tau_{e}^{-1}}$

3. $\sqrt{\operatorname{var}\left[\tilde{p}_{N}-\tilde{v}\right]} \propto 1 / \sqrt{N}$ and $\lim _{N \rightarrow \infty} \sqrt{N} \sqrt{\operatorname{var}\left[\tilde{p}_{N}-\tilde{v}\right]}=\sqrt{2 \tau_{e}^{-1}}$.

As in Proposition $8, \lim _{N \rightarrow \infty} \sqrt{N} \sqrt{\operatorname{var}\left[\tilde{p}_{N}-\tilde{v}\right]}$ is the largest among the asymptotic standard deviations.

\subsection{Informationally trivial limit}

Recall from Proposition 1 that if the number of informed speculators $N$ grows at a slower rate than noise trading $\sigma_{z}$ an informationally trivial limit obtains and prices converge to zero. Let us study the convergence rates in this case.

Proposition 10. For a sequence of markets with $\sigma_{z}$ growing faster than at the rate $N$ we obtain:

1. $\sqrt{\operatorname{var}\left[\tilde{p}_{N}-\tilde{p}_{N}^{\mathrm{c}}\right]} \propto N^{2} / \sigma_{z}^{3}(N)$;

2. $\sqrt{\operatorname{var}\left[\tilde{p}_{N}^{\mathrm{c}}\right]} \propto N / \sigma_{z}(N)$;

3. $\sqrt{\operatorname{var}\left[\tilde{p}_{N}\right]} \propto N / \sigma_{z}(N)$.

Therefore, the strategic effect vanishes faster than the limit effect as in our central scenario. 


\subsection{Summary table}

Table 3 summarizes the strategic and limit effects for prices in our central scenario and the cases considered in this section.

\begin{tabular}{|l|c|c|c|}
\hline & Limit price & Limit effect & Strategic effect \\
\hline$\sigma_{z} \sim O(\sqrt{N})$ & $\tilde{v}$, fully revealing & $1 / \sqrt{N}$ & $1 / \sqrt{N}$ \\
$\sqrt{N} \sim o\left(\sigma_{z}\right)$ and $\sigma_{z} \sim o(N)$ & $\tilde{v}$, fully revealing & $\sigma_{z} / N$ & $1 / \sigma_{z}$ \\
$\sigma_{z} \propto N$ & $\tilde{p}_{\infty}$ & $1 / \sqrt{N}$ & $1 / N$ \\
$N \sim o\left(\sigma_{z}\right)$ & 0 , informationally trivial & $N / \sigma_{z}$ & $N^{2} / \sigma_{z}^{3}$ \\
\hline
\end{tabular}

Table 3: Summary of the strategic and limit effects for prices.

One can see that whenever $\sqrt{N} \sim o\left(\sigma_{z}\right)$ the strategic effect is dominated by the limit effect in terms of the speed of convergence. In particular, this happens in our central scenario (the case when $\left.\sigma_{z} \propto N\right)$. When $\sigma_{z} \sim O(\sqrt{N})$ the speed of convergence is the same for both effects $(1 / \sqrt{N})$, but the strategic effect is still dominated by the limit effect in terms of the asymptotic variances.

\section{Traders close to risk neutral}

In this section we examine the limiting behavior of equilibria in the set-up of Section 3 when traders are close to risk neutrality $(\rho \rightarrow 0)$. For all of the markets $\operatorname{var}[\tilde{v}]=\tau_{v}^{-1}, \operatorname{var}\left[\tilde{e}_{n}\right]=\tau_{e}^{-1}$ and $\sqrt{\operatorname{var}[\tilde{z}]}=\sigma_{z}$. Allow traders to become informed by paying a fee $F\left(\sigma_{z}\right)>0$. Assume that $F(\infty) \geq 0$ is well defined.

Proposition 11. With a cost of acquiring information $F\left(\sigma_{z}\right)>0$, the asymptotic behavior of the endogenous number of informed speculators as $\rho \rightarrow 0$ is as follows:

1. $\left(\lim _{\rho \rightarrow 0} N^{*}\left(\sigma_{z}\right)\right) \propto\left(\sigma_{z} / F\left(\sigma_{z}\right)\right)^{2 / 3}$ in the strategic model; and

2. $\left(\lim _{\rho \rightarrow 0} N_{\mathrm{c}}^{*}\left(\sigma_{z}\right)\right)=0$ in the competitive model.

For example, in the central case of constant returns to information acquisition, $F(\cdot) \equiv$ $F>0$ we obtain $\lim _{\rho \rightarrow 0} N^{*}\left(\sigma_{z}\right) \propto\left(\sigma_{z}\right)^{2 / 3}$ for $\sigma_{z}$ large, while $\lim _{\rho \rightarrow 0} N_{\mathrm{c}}^{*}\left(\sigma_{z}\right)=0$. No traders choose to become informed in the price-taking case because of their closeness to risk neutrality. If they chose to become informed they would trade so aggressively that they would (almost) reveal their private information and would make (close to) zero profits. This is an example of the well-known informational efficiency paradox described by Grossman and Stiglitz (1980). In the strategic case the informed traders take into account the effect of their actions on the price and therefore can restrict their trade. So the incentives to acquire information do 
not disappear as more traders decide to become informed. It is worth noting that in the limit as $\rho \rightarrow 0$, unlike the risk-averse case, the number of informed traders grows less than proportionally with the size of the market $\sigma_{z}$. The reason is that risk neutrality implies fiercer competition among informed traders.

\section{Monopolistic competition}

We present here the "monopolistic competition" example of Kyle (1989), where the competitive and the strategic equilibrium are far apart even in a large market.

Consider a sequence of markets with the "monopolistic competition" limit in Kyle (1989, Section 9). At the $N$ th market there are $N$ informed agents and for all of the markets $\operatorname{var}[\tilde{v}]=\tau_{v}^{-1}$ is a fixed constant. However, now $\operatorname{var}\left[\tilde{e}_{n}\right]=\tau_{e}^{-1}$ and $N \tau_{e}=\tau_{E}$, where $\tau_{E}$ is a given constant. This means that, unlike in the previous sections, the total precision is bounded and does not change with $N$. A given stock of private information $\tau_{E}$ is divided equally among $N$ speculators. Thus, for large $N$ each speculator's signal contains only a small amount of information. It is convenient to define an average error term $\tilde{e} \equiv(1 / N) \sum_{n=1}^{N} \tilde{e}_{n}$. Obviously, $\tilde{e}$ is normally distributed with zero mean and $\operatorname{var}[\tilde{e}]=\left(N \tau_{e}\right)^{-1}=\tau_{E}^{-1}$.

It is easy to see that if $\operatorname{var}[\tilde{z}]=\sigma_{z}^{2}$ grows with $N$ and $\tau_{E}$ is fixed, then both the competitive and the strategic equilibrium reach the informationally trivial limit and equilibrium prices converge to zero.

Another interesting case appears when $\operatorname{var}[\tilde{z}]=\sigma_{z}^{2}$ is a fixed constant. First, let us demonstrate that if we endogenize the number of informed agents, by allowing any trader to be informed by paying a fixed amount $F>0$, then the number of informed in both the competitive and the strategic regime will be finite. (Note that we keep the noise trade constant in this model, so $F$ does not depend on $\sigma_{z}$.)

Proposition 12. In the monopolistic competition model with a cost of $F>0$ for becoming an informed trader, the endogenous number of informed speculators is finite in both the strategic and competitive cases. As $F \rightarrow 0$, both numbers tend to infinity.

The result follows because the certainty equivalent of profits in both the strategic and competitive cases tend to zero as $N$ grows. Now we confirm that the limits of the competitive and monopolistically competitive equilibria as $F \rightarrow 0$ are different, and that the convergence to the respective limit equilibria is fast, at a rate of at least $1 / N$, in both cases. The difference in the limits is due to the residual market power traders enjoy in the monopolistic competition case even in a large market. For the competitive model we show that the convergence is always at a rate of $1 / N$, while for the monopolistically competitive model we can show that $1 / N$ is the exact rate of convergence for the subcase $\tau_{E}>\tau_{v}$. Convergence is fast now because all along the sequence of markets, noise trade is constant and the average noise in the signal $\tilde{e}$ 
has constant variance. In contrast, in the central scenario we have that in the $N$ th market $\operatorname{var}[\tilde{e}]$ is of the order of $1 / N$ and this determines the convergence speed to the limit of $1 / \sqrt{N}$.

Proposition 13. For the sequence of markets with the monopolistic competition limit as $F \rightarrow 0$, different prices are obtained in the price-taking $\left(\tilde{p}_{\infty}^{\mathrm{c}}\right)$ and in the strategic case $\left(\tilde{p}_{\infty}\right)$ $\tilde{p}_{\infty} \neq \tilde{p}_{\infty}^{\mathrm{c}}$. Furthermore:

1. $\sqrt{\operatorname{var}\left[\tilde{p}_{N}^{\mathrm{c}}-\tilde{p}_{\infty}^{\mathrm{c}}\right]} \propto 1 / N ;$ and

2. $\sqrt{\operatorname{var}\left[\tilde{p}_{N}-\tilde{p}_{\infty}\right]} \sim O(1 / N)$ and if $\tau_{E}>\tau_{v}$, then $\sqrt{\operatorname{var}\left[\tilde{p}_{N}-\tilde{p}_{\infty}\right]} \propto 1 / N$.

\section{Concluding remarks}

The basic insight of the paper is that provided there is no residual market power in a large market, competitive and strategic equilibria should be close whenever price-taking traders have incentives to be restrained in their trading. This insight should be robust to dynamic considerations. Think, for example, of the extreme case of risk neutral traders. Then the same logic as in the static model would lead us to conclude that competitive traders in a multiperiod market will have no incentives to acquire information, since profits would be dissipated, while strategic traders would. In any case, an interesing extension of the model would be to consider multiperiod trading.

\section{Appendix}

\subsection{Characterization of the equilibria}

For the convenience of the reader we summarize here some characterization results from Kyle (1989). Note that our restriction of the competitive risk-neutral market making sector corresponds to the free entry of uninformed speculators $(M=\infty)$ in Kyle (1989).

For any symmetric linear equilibrium with the strategies $X_{n}=\mu+\beta i_{n}-\gamma p, n=1, \ldots, N$, we can solve the market clearing condition for the equilibrium price $\tilde{p}$. Then we obtain the result that $\tilde{p}$ is informationally equivalent to $\left(\sum_{n=1}^{N} X_{n}\left(\tilde{p}, \tilde{\imath}_{n}\right)+\tilde{z}\right)$ and therefore to $\left(\beta \sum_{n=1}^{N} \tilde{\imath}_{n}+\tilde{z}\right)$. Thus we can express the price in terms of the parameter $\beta$ :

$$
\begin{aligned}
\tilde{p} & =E[\tilde{v} \mid \tilde{p}]=E\left[\tilde{v} \mid \beta \sum_{n=1}^{N} \tilde{\imath}_{n}+\tilde{z}\right]=E\left[\tilde{v} \mid \beta \tilde{v} N+\beta \sum_{n=1}^{N} \tilde{e}_{n}+\tilde{z}\right] \\
& =\frac{\beta \tau_{v}^{-1} N}{\beta^{2} \tau_{v}^{-1} N^{2}+\beta^{2} \tau_{e}^{-1} N+\sigma_{z}^{2}}\left(\beta \tilde{v} N+\beta \sum_{n=1}^{N} \tilde{e}_{n}+\tilde{z}\right) .
\end{aligned}
$$


Regardless of the particular equilibrium concept, the linearity and symmetry assumptions allow useful measures of the informativeness of prices to be obtained. Define $\tau_{\mathrm{F}}$ as the precision of the forecast of the liquidation value $\tilde{v}$ based on all the information, that is $\tau_{\mathrm{F}}=\operatorname{var}^{-1}\left[\tilde{v} \mid \tilde{\imath}_{1}, \ldots, \tilde{\imath}_{N}\right]$. The assumptions made on the distributions of $\tilde{v}, \tilde{e}_{1}, \ldots, \tilde{e}_{N}$ imply that $\tau_{\mathrm{F}}=\tau_{v}+N \tau_{e}$. Now let us define the precision $\tau_{\mathrm{U}}$ that speculators have basing only on

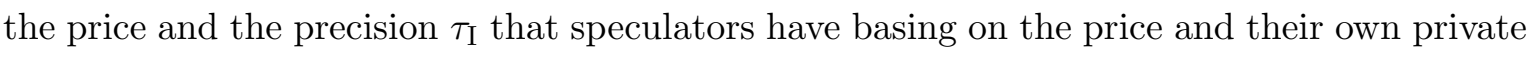
signal:

$$
\tau_{\mathrm{U}}=\operatorname{var}^{-1}[\tilde{v} \mid \tilde{p}] \quad \text { and } \quad \tau_{\mathrm{I}}=\operatorname{var}^{-1}\left[\tilde{v} \mid \tilde{p}, \tilde{\imath}_{n}\right]
$$

Normality makes $\tau_{\mathrm{U}}$ and $\tau_{\mathrm{I}}$ constants, while symmetry means that $\tau_{\mathrm{I}}$ does not depend on $n$. Since these precisions are bounded below by the prior precision $\tau_{v}$, and above by the full-information precision $\tau_{\mathrm{F}}$, there exist constants $\varphi_{\mathrm{U}}$ and $\varphi_{\mathrm{I}}$ both in the interval $[0,1]$, such that

$$
\tau_{\mathrm{U}}=\tau_{v}+\varphi_{\mathrm{U}} N \tau_{e} \quad \text { and } \quad \tau_{\mathrm{I}}=\tau_{v}+\tau_{e}+\varphi_{\mathrm{I}}(N-1) \tau_{e}
$$

The parameters $\varphi_{\mathrm{U}}$ and $\varphi_{\mathrm{I}}$ are convenient indices measuring the "informational efficiency" with which price aggregate private information of informed traders. Theorem 4.1 in Kyle (1989) presents expressions for these indices in terms of the parameter $\beta$ :

$$
\varphi_{\mathrm{I}}=\frac{(N-1) \beta^{2}}{(N-1) \beta^{2}+\sigma_{z}^{2} \tau_{e}} \quad \text { and } \quad \varphi_{\mathrm{U}}=\frac{N \beta^{2}}{N \beta^{2}+\sigma_{z}^{2} \tau_{e}} .
$$

These formulas imply

$$
\varphi_{\mathrm{U}}-\varphi_{\mathrm{I}}=\frac{1}{N} \varphi_{\mathrm{U}}\left(1-\varphi_{\mathrm{I}}\right)
$$

Let us turn back now to the two specific equilibria that we are studying. From (1) we obtain expressions for the prices in competitive and strategic equilibria:

$$
\begin{aligned}
\tilde{p}^{\mathrm{c}} & =\frac{\beta^{\mathrm{c}} \tau_{v}^{-1} N}{\left(\beta^{\mathrm{c}}\right)^{2} \tau_{v}^{-1} N^{2}+\left(\beta^{\mathrm{c}}\right)^{2} \tau_{e}^{-1} N+\sigma_{z}^{2}}\left(\beta^{\mathrm{c}} \tilde{v} N+\beta^{\mathrm{c}} \sum_{n=1}^{N} \tilde{e}_{n}+\tilde{z}\right), \\
\tilde{p} & =\frac{\beta \tau_{v}^{-1} N}{\beta^{2} \tau_{v}^{-1} N^{2}+\beta^{2} \tau_{e}^{-1} N+\sigma_{z}^{2}}\left(\beta \tilde{v} N+\beta \sum_{n=1}^{N} \tilde{e}_{n}+\tilde{z}\right) .
\end{aligned}
$$

The only difference in the expressions for prices is the difference between the parameters $\beta$ and $\beta^{\text {c }}$. Theorem 5.2 and expression (C.5) in Kyle (1989) provide a useful characterization of these parameters:

$$
\beta^{\mathrm{c}}=\frac{\tau_{e}}{\rho}\left(1-\varphi_{\mathrm{I}}^{\mathrm{c}}\right) \quad \text { and } \quad \beta=\frac{\tau_{e}}{\rho}\left(1-\varphi_{\mathrm{I}}\right) \frac{(1-2 \zeta)}{(1-\zeta)}
$$


where $\zeta=\tau_{\mathrm{I}} \beta \lambda / \tau_{e} \leq 1 / 2$, and

$$
(1-\zeta)=\left(1-\xi_{\mathrm{I}}\right)\left(1-\varphi_{\mathrm{I}}\right) \quad \text { and } \quad 0 \leq \xi_{\mathrm{I}} \leq 1 / N
$$

where $\xi_{\mathrm{I}}=\gamma \lambda$ can be interpreted as the marginal market share of an informed speculator. Condition (64) in Kyle (1989) will be true for $\zeta$ in our case:

$$
\zeta \tau_{\mathrm{U}}-\varphi_{\mathrm{U}} \tau_{\mathrm{I}}=0
$$

Finally, the following characterization (Lemma 7.1 of Kyle (1989)) of $\varphi_{\mathrm{I}}$ and $\varphi_{\mathrm{I}}^{\mathrm{c}}$ in the corresponding equilibria is very useful:

$$
\begin{aligned}
& \frac{\sigma_{z}^{2} \rho^{2}}{(N-1) \tau_{e}}=\frac{\left(1-\varphi_{\mathrm{I}}^{\mathrm{c}}\right)^{3}}{\varphi_{\mathrm{I}}^{\mathrm{c}}} \\
& \frac{\sigma_{z}^{2} \rho^{2}}{(N-1) \tau_{e}}=\frac{\left(1-\varphi_{\mathrm{I}}\right)^{3}}{\varphi_{\mathrm{I}}} \frac{(1-2 \zeta)^{2}}{(1-\zeta)^{2}}=\frac{\left(1-\varphi_{\mathrm{I}}\right)}{\varphi_{\mathrm{I}}} \frac{(1-2 \zeta)^{2}}{\left(1-\xi_{\mathrm{I}}\right)^{2}}
\end{aligned}
$$

\subsection{Limit marginal market shares}

One conclusion in Theorem 9.2 in Kyle (1989) requires some qualification for the case of free entry of uninformed speculators. Theorem 9.2 in Kyle (1989) covers the case where the limit is of the Hellwig-Admati type (as in Proposition 2) and concludes that $\xi_{\mathrm{U}} M \rightarrow 0$, which is equivalent to $\xi_{\mathrm{I}} N \rightarrow 1$. However, if we restrict attention to the case of a competitive risk-neutral market making sector or free entry of uninformed speculators $(M=\infty)$, then Lemma 3 below proves that for the case where the limit is of the Hellwig-Admati type,

$$
\lim _{N \rightarrow \infty} \xi_{\mathrm{I}} N=\frac{\tau_{e}^{2}}{\tau_{v} \sigma_{z_{0}}^{2} \rho^{2}+\tau_{e}^{2}}<1
$$

Note that parameters $\xi_{\mathrm{U}}$ and $\xi_{\mathrm{I}}$ can be interpreted respectively as marginal market shares of an uninformed speculator and of an informed speculator. Thus, the total marginal market share of all informed speculators $\left(\xi_{\mathrm{I}} N\right)$ even in the limit is strictly less than one, and it is close to one only if the precision of their signals $\left(\tau_{e}\right)$ is large relative to the other parameters of the model.

Similarly, Theorem 9.1 in Kyle (1989) predicts for the case of monopolistic competition that $\xi_{\mathrm{I}} N \rightarrow 1$. Again, for $M=\infty$ this does not hold. Lemma 5 below proves that in our case with a competitive risk-neutral market making sector, or free entry of uninformed speculators $(M=\infty)$, and monopolistic competition

$$
\xi_{\mathrm{I}} N=\frac{\tau_{v}+\tau_{E}}{\left(\tau_{v} / \varphi_{\mathrm{U}}\right)+\tau_{E}} .
$$

Since $\lim _{N \rightarrow \infty} \varphi_{\mathrm{U}}<1$, we can conclude that the total marginal market share of all 
informed speculators $\left(\xi_{\mathrm{I}} N\right)$ even in the limit is strictly less than one.

\subsection{Proofs}

Proof of Proposition 1 The proof proceeds in three steps. We deal in some detail with the case of the competitive equilibrium. The case of the strategic equilibrium follows similarly.

Step 1. Let us start with an exogenously given number of informed speculators $N>0$ and let us compare $\Pi^{\mathrm{c}}(N)$ and $F^{*}$. Kyle $(1989$, Theorem 10.1) provides the following expression for the certainty equivalents of profits in the competitive case:

$$
\Pi^{\mathrm{c}}(N)=\frac{1}{2 \rho} \log \left(1+\frac{\left(1-\varphi_{\mathrm{I}}^{\mathrm{c}}\right)\left(1-\varphi_{\mathrm{U}}^{\mathrm{c}}\right) \tau_{e}}{\tau_{\mathrm{U}}^{\mathrm{c}}}\right)
$$

To simplify notation, let us define

$$
\phi^{\mathrm{c}}\left(\sigma_{z}, N\right) \equiv \frac{\left(1-\varphi_{\mathrm{I}}^{\mathrm{c}}\right)\left(1-\varphi_{\mathrm{U}}^{\mathrm{c}}\right) \tau_{e}}{\tau_{\mathrm{U}}^{\mathrm{c}}}=\frac{\left(1-\varphi_{\mathrm{I}}^{\mathrm{c}}\right)\left(1-\varphi_{\mathrm{U}}^{\mathrm{c}}\right) \tau_{e}}{\tau_{v}+\varphi_{\mathrm{U}}^{\mathrm{c}} N \tau_{e}}=\frac{\left(1-\varphi_{\mathrm{I}}^{\mathrm{c}}\right)\left(1-\varphi_{\mathrm{U}}^{\mathrm{c}}\right)}{1+\varphi_{\mathrm{U}}^{\mathrm{c}} N \tau_{e} \tau_{v}^{-1}} \frac{\tau_{e}}{\tau_{v}} .
$$

Since $\left(1-\varphi_{\mathrm{I}}^{\mathrm{c}}\right)\left(1-\varphi_{\mathrm{U}}^{\mathrm{c}}\right) \leqslant 1$ and $1+\varphi_{\mathrm{U}}^{\mathrm{c}} N \tau_{e} \tau_{v}^{-1} \geqslant 1$ we have that $0 \leqslant \phi^{\mathrm{c}}\left(\sigma_{z}, N\right) \leqslant \tau_{e} / \tau_{v}$ and, therefore,

$$
0 \leqslant \Pi^{\mathrm{c}}(N) \leqslant \frac{1}{2 \rho} \log \left(1+\frac{\tau_{e}}{\tau_{v}}\right)=F^{*} .
$$

Step 2. Now let us consider various cases of possible growth of $\sigma_{z}$ and $N$ and their effect on $\Pi^{\mathrm{c}}(N)$ :

Case 1. If $N$ grows slower than at the rate $\sigma_{z}^{2}$, then the left-hand side of characterization (8) increases at a rate of $\sigma_{z}^{2} / N$. Therefore, the right-hand side of characterization (8) also increases at a rate of $\sigma_{z}^{2} / N$. However, this is only possible if $\varphi_{\mathrm{I}}^{\mathrm{c}}$ converges to zero at a rate of $N / \sigma_{z}^{2}$. Then it follows from (3) that $\varphi_{\mathrm{U}}^{\mathrm{c}}$ also converges to zero. Moreover, then the same condition (3) implies that $\varphi_{\mathrm{U}}^{\mathrm{c}}$ converges to zero also at a rate of $N / \sigma_{z}^{2}$. The following subcases of this case should be separated.

Subcase (a) of case 1). If $N$ grows slower than at the rate $\sigma_{z}$, then we have that $\tau_{\mathrm{U}}^{\mathrm{c}}=$ $\tau_{v}+\varphi_{\mathrm{U}}^{\mathrm{c}} N \tau_{e}$ converges to $\tau_{v}$. So, the limit model is informationally trivial. Moreover $\phi^{\mathrm{c}}\left(\sigma_{z}, N\right)$ converges to $\tau_{e} / \tau_{v}$ and $\Pi^{\mathrm{c}}(N)$ converges to $F^{*}$.

Subcase (b) of case 1). If $N$ grows at the same rate as $\sigma_{z}$, that is $\sigma_{z} \propto N$, then it follows from (8) that $\varphi_{\mathrm{I}}^{\mathrm{c}} \propto N^{-1}$. This implies from (3) that $\varphi_{\mathrm{U}}^{\mathrm{c}} \propto N^{-1}$. Hence, there exist positive constants $k_{1}$ and $k_{2}$ such that $k_{1} \leqslant \varphi_{\mathrm{U}}^{\mathrm{c}} N \leqslant k_{2}$ for all large $N$. Therefore, there exist constants $c_{1}$ and $c_{2}$ such that $0<c_{1} \leqslant \phi^{\mathrm{c}}\left(\sigma_{z}, N\right) \leqslant c_{2}<\tau_{e} / \tau_{v}$ for all large $N$. Thus, there exist 
constants $F_{1}$ and $F_{2}$ such that $0<F_{1} \leqslant \Pi^{\mathrm{c}}(N) \leqslant F_{2}<F^{*}$ for all large $N$. In this case it is not possible that $\Pi^{\mathrm{c}}(N)$ converges to zero or $F^{*}$. Note that $\Pi^{\mathrm{c}}(N)$ would converge to some constant $F^{\prime}, 0<F^{\prime}<F^{*}$ and the limit model will be well defined if $\sigma_{z} / N$ converges to some positive number. (If $\sigma_{z} / N$ converges to some positive number, then by (8) $\varphi_{\mathrm{I}}^{\mathrm{c}} N$ converges to some positive number. So, by $(3), \varphi_{\mathrm{U}}^{\mathrm{c}} N$ converges to the same positive number as $\varphi_{\mathrm{I}}^{\mathrm{c}} N$. Then $\phi^{\mathrm{c}}\left(\sigma_{z}, N\right)$ converges to some constant $c^{\prime}, 0<c^{\prime}<\tau_{e} / \tau_{v}$. Hence, $\Pi^{\mathrm{c}}(N)$ would also converge to some constant $F^{\prime}, 0<F^{\prime}<F^{*}$.) If the limit model is well defined, the limit will be neither value revealing nor informationally trivial.

Subcase (c) of case 1). Now let us consider the case that $N$ grows faster than at the rate $\sigma_{z}$, but slower than at the rate $\sigma_{z}^{2}$. Then $\tau_{\mathrm{U}}^{\mathrm{c}}=\tau_{v}+\varphi_{\mathrm{U}}^{\mathrm{c}} N \tau_{e}$ increases to infinity at the rate $N^{2} / \sigma_{z}^{2}$. Therefore, $\phi^{\mathrm{c}}\left(\sigma_{z}, N\right)$ and $\Pi^{\mathrm{c}}(N)$ converge to zero. The limit will be value revealing. (Recall that $E\left[\tilde{v} \mid \tilde{p}_{N}^{\mathrm{c}}\right]=\tilde{p}_{N}^{\mathrm{c}}$. This implies that $\operatorname{var}\left[\tilde{v}-\tilde{p}_{N}^{\mathrm{c}}\right]=\operatorname{var}\left[\tilde{v} \mid \tilde{p}_{N}^{\mathrm{c}}\right]=\left(\tau_{\mathrm{U}}^{\mathrm{c}}\right)^{-1}$ and, thus, converges to zero.)

Case 2. If $N$ grows at a rate $\sigma_{z}^{2}$ or faster, then the left-hand side of characterization (8) is bounded by some number. Therefore, the right-hand side of characterization (8) is also bounded by this number. Therefore, $\varphi_{\mathrm{I}}^{\mathrm{c}}$ does not converge to zero. Then it follows from (3) that $\varphi_{\mathrm{U}}^{\mathrm{c}}$ also does not converge to zero. Since $\varphi_{\mathrm{U}}^{\mathrm{c}} \leqslant 1$ we get that $\left(\tau_{\mathrm{U}}^{\mathrm{c}}\right)_{N}=\tau_{v}+\varphi_{\mathrm{U}}^{\mathrm{c}} N \tau_{e}$ increases to infinity at the rate $N$. Therefore $\phi^{\mathrm{c}}\left(\sigma_{z}, N\right)$ and $\Pi^{\mathrm{c}}(N)$ converge to zero. The limit will be value revealing. (Recall again that $E\left[\tilde{v} \mid \tilde{p}_{N}^{\mathrm{c}}\right]=\tilde{p}_{N}^{\mathrm{c}}$. This implies that $\operatorname{var}\left[\tilde{v}-\tilde{p}_{N}^{\mathrm{c}}\right]=\operatorname{var}\left[\tilde{v} \mid \tilde{p}_{N}^{\mathrm{c}}\right]=\left(\tau_{\mathrm{U}}^{\mathrm{c}}\right)^{-1}$ and thus converges to zero. $)$

Summarizing all of the cases: $\Pi^{\mathrm{c}}(N)$ converges to $F^{*}$ if $N$ grows slower than at the rate $\sigma_{z}$ (note that this covers the case when $N$ does not grow at all), case 1(a) above; $0<F_{1} \leqslant \Pi^{\mathrm{c}}(N) \leqslant F_{2}<F^{*}$ for all large $N$ if $N$ grows at the same rate as $\sigma_{z}$, case $1(\mathrm{~b})$ above; and $\Pi^{\mathrm{c}}(N)$ converges to zero if $N$ grows faster than at the rate $\sigma_{z}$, cases $1(\mathrm{c})$ and 2 above. Moreover, note that $N$ and $N+1$ always grow at the same rate with respect to $\sigma_{z} \rightarrow \infty$ and none of the limits above change with a substitution of $N+1$ in place of $N$. We can thus conclude that $\Pi^{\mathrm{c}}(N+1)$ always converges to the same limit as $\Pi^{\mathrm{c}}(N)$.

Step 3. Now let us turn to the endogenously determined number of informed speculators $N_{\mathrm{c}}^{*}\left(\sigma_{z}\right)$. Recall that it is defined so that

$$
\Pi^{\mathrm{c}}\left(N_{\mathrm{c}}^{*}\left(\sigma_{z}\right)\right) \geq F\left(\sigma_{z}\right)>\Pi^{\mathrm{c}}\left(N_{\mathrm{c}}^{*}\left(\sigma_{z}\right)+1\right) .
$$

Thus, as in Step 2, $\Pi^{\mathrm{c}}\left(N_{\mathrm{c}}^{*}\left(\sigma_{z}\right)\right)$ and $\Pi^{\mathrm{c}}\left(N_{\mathrm{c}}^{*}\left(\sigma_{z}\right)+1\right)$ should converge to the same limit. For $F(\infty) \leq F^{*}$ we can conclude that $\Pi^{\mathrm{c}}\left(N_{\mathrm{c}}^{*}\left(\sigma_{z}\right)\right) \rightarrow F(\infty)$. For $F(\infty)>F^{*}$ we must have 
$N_{\mathrm{c}}^{*}\left(\sigma_{z}\right)=0$ for all large $\sigma_{z}$. Three cases are now possible.

(I) If $F(\infty) \geq F^{*}$, then either $F(\infty)>F^{*}$ and $N_{c}^{*}\left(\sigma_{z}\right)=0$ for all large $\sigma_{z}$ or $F(\infty)=F^{*}$ and by the exclusion of cases $1(\mathrm{~b}), 1(\mathrm{c})$ and 2 we must be in the case 1 (a) above. In either variant $N_{\mathrm{c}}^{*}\left(\sigma_{z}\right) \sim o\left(\sigma_{z}\right)$ and the limit market is informationally trivial. (Note that if $F(\cdot) \equiv F>0, N>0$ speculators can chose to become informed only if $\Pi^{\mathrm{c}}(N) \geqslant F$. For $F>F^{*}$ this is not possible since $\Pi^{\mathrm{c}}(N)$ never exceeds $F^{*}$ for any $N$. For $F=F^{*}$ this would require $\varphi_{\mathrm{I}}^{\mathrm{c}}=\varphi_{\mathrm{U}}^{\mathrm{c}}=0$. Then by (5) it will imply that $\beta^{\mathrm{c}}=\tau_{e} / \rho$. However, then our assumption that $N>0$ makes the second formula of (2) impossible to hold. A contradiction. Hence, $N_{\mathrm{c}}^{*}\left(\sigma_{z}\right)=0$.)

(II) If $0<F(\infty)<F^{*}$, then by the exclusion of cases $1(\mathrm{a}), 1(\mathrm{c})$ and 2 only the case 1 (b) above is possible. Therefore, $N_{\mathrm{c}}^{*}\left(\sigma_{z}\right) \propto \sigma_{z}$ and both $\varphi_{\mathrm{I}}^{\mathrm{c}}$ and $\varphi_{\mathrm{U}}^{\mathrm{c}}$ converge to zero. Moreover, since $\Pi^{\mathrm{c}}\left(N_{\mathrm{c}}^{*}\left(\sigma_{z}\right)\right) \rightarrow F(\infty)$ and

$$
\Pi^{\mathrm{c}}(N)=\frac{1}{2 \rho} \log \left(1+\frac{\left(1-\varphi_{\mathrm{I}}^{\mathrm{c}}\right)\left(1-\varphi_{\mathrm{U}}^{\mathrm{c}}\right) \tau_{e}}{\tau_{\mathrm{U}}^{\mathrm{c}}}\right),
$$

we have that $\tau_{\mathrm{U}}^{\mathrm{c}} \rightarrow \tau_{e} /(\exp (2 \rho F(\infty))-1)$. Therefore, since $\tau_{\mathrm{U}}^{\mathrm{c}}=\tau_{v}+\varphi_{\mathrm{U}}^{\mathrm{c}} N \tau_{e}$, we obtain that $\varphi_{\mathrm{U}}^{\mathrm{c}} N_{\mathrm{c}}^{*}\left(\sigma_{z}\right) \rightarrow 1 /(\exp (2 \rho F(\infty))-1)-\tau_{v} / \tau_{e}$. Then by $(3) \varphi_{\mathrm{I}}^{\mathrm{c}} N_{\mathrm{c}}^{*}\left(\sigma_{z}\right) \rightarrow$ $1 /(\exp (2 \rho F(\infty))-1)-\tau_{v} / \tau_{e}$ and, by $(8)$,

$$
\frac{\sigma_{z}}{N_{\mathrm{c}}^{*}\left(\sigma_{z}\right)} \rightarrow \frac{1}{\rho} \sqrt{\frac{\tau_{e}}{1 /(\exp (2 \rho F(\infty))-1)-\tau_{v} / \tau_{e}}} .
$$

So, the limit market is well defined and is neither value revealing nor informationally trivial.

(III) If $F(\infty)=0$, then by the exclusion of cases 1 (a) and 1(b) only cases 1(c) or 2 above are possible. Therefore, $\sigma_{z} \sim o\left(N_{\mathrm{c}}^{*}\left(\sigma_{z}\right)\right)$ and the limit market is value revealing. (Note that if $F(\cdot) \equiv F=0$, then obviously $N_{\mathrm{c}}^{*}\left(\sigma_{z}\right)=\infty$.)

Now let us briefly consider the case of the strategic equilibrium. The proof in this case goes along the same lines as in the competitive case. Kyle (1989, Theorem 10.1) provides the following expression for the certainty equivalents of profits in strategic case:

$$
\Pi(N)=\frac{1}{2 \rho} \log \left(1+\frac{\left(1-\varphi_{\mathrm{I}}\right)\left(1-\varphi_{\mathrm{U}}\right) \tau_{e}}{\tau_{\mathrm{U}}} \frac{(1-2 \zeta)}{(1-\zeta)^{2}}\right) .
$$

We can similarly define

$$
\phi\left(\sigma_{z}, N\right) \equiv \frac{\left(1-\varphi_{\mathrm{I}}\right)\left(1-\varphi_{\mathrm{U}}\right) \tau_{e}}{\tau_{\mathrm{U}}} \frac{(1-2 \zeta)}{(1-\zeta)^{2}}=\frac{\left(1-\varphi_{\mathrm{I}}\right)\left(1-\varphi_{\mathrm{U}}\right)}{1+\varphi_{\mathrm{U}} N \tau_{e} \tau_{v}^{-1}} \frac{(1-2 \zeta)}{(1-\zeta)^{2}} \frac{\tau_{e}}{\tau_{v}} .
$$

Since $\left(1-\varphi_{\mathrm{I}}\right)\left(1-\varphi_{\mathrm{U}}\right) \leqslant 1,(1-2 \zeta) \leqslant(1-\zeta)^{2}$ and $1+\varphi_{\mathrm{U}} N \tau_{e} \tau_{v}^{-1} \geqslant 1$, we have $0 \leqslant \Pi(N) \leqslant F^{*}$ 
for any $N$. Then an analysis analogous to steps $1-3$ above completes the proof.

Proof of Proposition 2 From the formulas (4) we obtain the following expressions for prices in both equilibria in the $N$-replica market:

$$
\begin{aligned}
& \tilde{p}_{N}^{\mathrm{c}}=\frac{\beta_{N}^{\mathrm{c}} \tau_{v}^{-1}}{\left(\beta_{N}^{\mathrm{c}}\right)^{2} \tau_{v}^{-1}+\frac{1}{N}\left(\beta_{N}^{\mathrm{c}}\right)^{2} \tau_{e}^{-1}+\sigma_{z_{0}}^{2}}\left(\beta_{N}^{\mathrm{c}} \tilde{v}+\beta_{N}^{\mathrm{c}} \frac{1}{N} \sum_{n=1}^{N} \tilde{e}_{n}+\tilde{z}_{0}\right), \\
& \tilde{p}_{N}=\frac{\beta_{N} \tau_{v}^{-1}}{\beta_{N}^{2} \tau_{v}^{-1}+\frac{1}{N} \beta_{N}^{2} \tau_{e}^{-1}+\sigma_{z_{0}}^{2}}\left(\beta_{N} \tilde{v}+\beta_{N} \frac{1}{N} \sum_{n=1}^{N} \tilde{e}_{n}+\tilde{z}_{0}\right) .
\end{aligned}
$$

The expression for the limiting price in this model is also well known (e.g. Vives (1995); alternatively it is a consequence of our proof below):

$$
\tilde{p}_{\infty}=\frac{\beta_{\infty} \tau_{v}^{-1}}{\beta_{\infty}^{2} \tau_{v}^{-1}+\sigma_{z_{0}}^{2}}\left(\beta_{\infty} \tilde{v}+\tilde{z}_{0}\right) \quad \text { where } \beta_{\infty}=\frac{\tau_{e}}{\rho}
$$

Let us prove first two lemmas.

Lemma 1. We have $\zeta_{N} \propto\left(\varphi_{\mathrm{I}}^{\mathrm{c}}\right)_{N} \propto\left(\varphi_{\mathrm{I}}\right)_{N} \propto 1 / N$ and $\left(\varphi_{\mathrm{I}}\right)_{N}-\left(\varphi_{\mathrm{I}}^{\mathrm{c}}\right)_{N} \propto 1 / N^{2}$.

Proof. The (equal) left-hand sides of (8) grow at the rate $N$. Thus, the right-hand sides of both expressions should grow at the rate $N$. Since $\left(\xi_{\mathrm{I}}\right)_{N} \leq 1 / N$ (see (6)), this implies that $\left(\varphi_{\mathrm{I}}^{\mathrm{c}}\right)_{N} \propto 1 / N$, and $\left(\varphi_{\mathrm{I}}\right)_{N} \propto 1 / N$. Since $(1-\zeta)=\left(1-\xi_{\mathrm{I}}\right)\left(1-\varphi_{\mathrm{I}}\right)($ see $(6))$, we have $\zeta \propto 1 / N$. Now from (8) it follows that

$$
\frac{\left(1-\varphi_{\mathrm{I}}^{\mathrm{c}}\right)^{3}}{\varphi_{\mathrm{I}}^{\mathrm{c}}}=\frac{\left(1-\varphi_{\mathrm{I}}\right)^{3}}{\varphi_{\mathrm{I}}} \frac{(1-2 \zeta)^{2}}{(1-\zeta)^{2}} .
$$

Therefore,

$$
\frac{\left(1-\varphi_{\mathrm{I}}^{\mathrm{c}}\right)^{3}}{\left(1-\varphi_{\mathrm{I}}\right)^{3}} \frac{\varphi_{\mathrm{I}}}{\varphi_{\mathrm{I}}^{\mathrm{c}}}-1=\frac{(1-2 \zeta)^{2}}{(1-\zeta)^{2}}-1
$$

Then

$$
\frac{(1-2 \zeta)^{2}}{(1-\zeta)^{2}}-1=\frac{\zeta(2-3 \zeta)}{(1-\zeta)^{2}} \propto 1 / N
$$

and

$$
\begin{aligned}
\frac{\left(1-\varphi_{\mathrm{I}}^{\mathrm{c}}\right)^{3}}{\left(1-\varphi_{\mathrm{I}}\right)^{3}} \frac{\varphi_{\mathrm{I}}}{\varphi_{\mathrm{I}}^{\mathrm{c}}}-1 & =\frac{\left(1-\varphi_{\mathrm{I}}^{\mathrm{c}}\right)^{3}}{\left(1-\varphi_{\mathrm{I}}\right)^{3}}\left(\frac{\varphi_{\mathrm{I}}}{\varphi_{\mathrm{I}}^{\mathrm{c}}}-1\right)+\left(\frac{\left(1-\varphi_{\mathrm{I}}^{\mathrm{c}}\right)^{3}}{\left(1-\varphi_{\mathrm{I}}\right)^{3}}-1\right) \\
& \propto N\left(\varphi_{\mathrm{I}}-\varphi_{\mathrm{I}}^{\mathrm{c}}\right)+O\left(\varphi_{\mathrm{I}}-\varphi_{\mathrm{I}}^{\mathrm{c}}\right) \propto N\left(\varphi_{\mathrm{I}}-\varphi_{\mathrm{I}}^{\mathrm{c}}\right) .
\end{aligned}
$$

Hence, $\left(\varphi_{\mathrm{I}}\right)_{N}-\left(\varphi_{\mathrm{I}}^{\mathrm{c}}\right)_{N} \propto 1 / N^{2}$.

Lemma 2. We have $\left(\beta_{N}^{\mathrm{c}}-\beta_{\infty}\right) \propto\left(\beta_{N}-\beta_{\infty}\right) \propto\left(\beta_{N}-\beta_{N}^{\mathrm{c}}\right) \propto 1 / N$. 
Proof. Recall from (5) that

$$
\beta^{\mathrm{c}}=\frac{\tau_{e}}{\rho}\left(1-\varphi_{\mathrm{I}}^{\mathrm{c}}\right) \quad \text { and } \quad \beta=\frac{\tau_{e}}{\rho}\left(1-\varphi_{\mathrm{I}}\right) \frac{(1-2 \zeta)}{(1-\zeta)} .
$$

Thus,

$$
\beta_{N}^{\mathrm{c}}-\beta_{\infty}=\frac{\tau_{e}}{\rho} \varphi_{\mathrm{I}}^{\mathrm{c}} \propto \frac{1}{N}
$$

Also

$$
1-\left(1-\varphi_{\mathrm{I}}\right) \frac{(1-2 \zeta)}{(1-\zeta)}=1-\left(1-\varphi_{\mathrm{I}}\right)+\left(1-\varphi_{\mathrm{I}}\right) \frac{\zeta}{(1-\zeta)}=\varphi_{\mathrm{I}}+\left(1-\varphi_{\mathrm{I}}\right) \frac{\zeta}{(1-\zeta)} \propto 1 / N
$$

Hence, $\beta_{N}-\beta_{\infty} \propto 1 / N$. From (2) we have

$$
\varphi_{\mathrm{I}}-\varphi_{\mathrm{I}}^{\mathrm{c}}=\frac{(N-1) \sigma_{z}^{2} \tau_{e}\left(\beta_{N}^{2}-\left(\beta_{N}^{\mathrm{c}}\right)^{2}\right)}{\left((N-1) \beta_{N}^{2}+\sigma_{z}^{2} \tau_{e}\right)\left((N-1)\left(\beta_{N}^{\mathrm{c}}\right)^{2}+\sigma_{z}^{2} \tau_{e}\right)} \propto \frac{N^{3}\left(\beta_{N}-\beta_{N}^{\mathrm{c}}\right)}{N^{4}} .
$$

Thus $\beta_{N}-\beta_{N}^{\mathrm{c}} \propto 1 / N$.

Now let us continue with the proof of Proposition 2. We use the notation $M^{\infty}:=$ $\beta_{\infty}^{2} \tau_{v}^{-1}+\sigma_{z_{0}}^{2}, M^{\mathrm{c}}:=\left(\beta_{N}^{\mathrm{c}}\right)^{2} \tau_{v}^{-1}+(1 / N)\left(\beta_{N}^{\mathrm{c}}\right)^{2} \tau_{e}^{-1}+\sigma_{z_{0}}^{2}$, and $M:=\beta_{N}^{2} \tau_{v}^{-1}+(1 / N) \beta_{N}^{2} \tau_{e}^{-1}+\sigma_{z_{0}}^{2}$. Then from (9) we obtain

$$
\tilde{p}_{N}-\tilde{p}_{N}^{\mathrm{c}}=a \tilde{v}+a \frac{1}{N} \sum_{n=1}^{N} \tilde{e}_{n}+b \tilde{z}_{0},
$$

where

$$
a:=\frac{\beta_{N}^{2} \tau_{v}^{-1}}{M}-\frac{\left(\beta_{N}^{\mathrm{c}}\right)^{2} \tau_{v}^{-1}}{M^{\mathrm{c}}} \text { and } b:=\frac{\beta_{N} \tau_{v}^{-1}}{M}-\frac{\beta_{N}^{\mathrm{c}} \tau_{v}^{-1}}{M^{\mathrm{c}}} .
$$

Then $a=\left(M M^{\mathrm{c}}\right)^{-1}\left(\left(\beta_{N}^{2}-\left(\beta_{N}^{\mathrm{c}}\right)^{2}\right) \tau_{v}^{-1} \sigma_{z_{0}}^{2}\right) \propto\left(\beta_{N}-\beta_{N}^{\mathrm{c}}\right) \propto 1 / N$ and $b=\left(M M^{\mathrm{c}}\right)^{-1}\left(\beta_{N} \beta_{N}^{\mathrm{c}}\left(\beta_{N}^{\mathrm{c}}-\right.\right.$ $\left.\left.\beta_{N}\right)\left(\tau_{v}^{-1}+(1 / N) \tau_{e}^{-1}\right) \tau_{v}^{-1}+\left(\beta_{N}-\beta_{N}^{\mathrm{c}}\right) \tau_{v}^{-1} \sigma_{z_{0}}^{2}\right)=O(1 / N)$. So

$$
\operatorname{var}\left[\tilde{p}_{N}-\tilde{p}_{N}^{\mathrm{c}}\right]=a^{2} \tau_{v}^{-1}+a^{2} \frac{1}{N} \tau_{e}^{-1}+b^{2} \sigma_{z_{0}}^{2} \propto 1 / N^{2} .
$$

Then from (9) and (10) we obtain

$$
\tilde{p}_{N}^{\mathrm{c}}-\tilde{p}_{\infty}=f \tilde{v}+g \frac{1}{N} \sum_{n=1}^{N} \tilde{e}_{n}+h \tilde{z}_{0},
$$

where

$$
f:=\frac{\left(\beta_{N}^{\mathrm{c}}\right)^{2} \tau_{v}^{-1}}{M^{\mathrm{c}}}-\frac{\beta_{\infty}^{2} \tau_{v}^{-1}}{M^{\infty}}, \quad g:=\frac{\left(\beta_{N}^{\mathrm{c}}\right)^{2} \tau_{v}^{-1}}{M^{\mathrm{c}}} \quad \text { and } \quad h:=\frac{\beta_{N}^{\mathrm{c}} \tau_{v}^{-1}}{M^{\mathrm{c}}}-\frac{\beta_{\infty} \tau_{v}^{-1}}{M^{\infty}} .
$$

Then $f=\left(M^{\mathrm{c}} M^{\infty}\right)^{-1}\left(\left(\left(\beta_{N}^{\mathrm{c}}\right)^{2}-\beta_{\infty}^{2}\right) \tau_{v}^{-1} \sigma_{z_{0}}^{2}-\beta_{\infty}^{2} \tau_{v}^{-1}(1 / N)\left(\beta_{N}^{\mathrm{c}}\right)^{2} \tau_{e}^{-1}\right)=O(1 / N)$ and $h=$ $\left(M^{\mathrm{c}} M^{\infty}\right)^{-1}\left(\beta_{N}^{\mathrm{c}} \beta_{\infty}\left(\beta_{\infty}-\beta_{N}^{\mathrm{c}}\right)\left(\tau_{v}^{-1}\right)^{2}+\left(\beta_{N}^{\mathrm{c}}-\beta_{N}\right) \tau_{v}^{-1} \sigma_{z_{0}}^{2}-\beta_{\infty}^{2} \tau_{v}^{-1}(1 / N)\left(\beta_{N}^{\mathrm{c}}\right)^{2} \tau_{e}^{-1}\right)=O(1 / N)$. 
Finally, $g$ converges to the constant. Thus,

$$
\operatorname{var}\left[\tilde{p}_{N}^{\mathrm{c}}-\tilde{p}_{\infty}\right]=f^{2} \tau_{v}^{-1}+g^{2} \frac{1}{N} \tau_{e}^{-1}+h^{2} \sigma_{z_{0}}^{2} \propto 1 / N
$$

Proof of Proposition 3 We follow the notation from the proof of Proposition 2.

1. It appears from the proof of Proposition 2 that

$$
\lim _{N \rightarrow \infty} N \operatorname{var}\left[\tilde{p}_{N}^{\mathrm{c}}-\tilde{p}_{\infty}\right]=\lim _{N \rightarrow \infty} g^{2} \tau_{e}^{-1}=\lim _{N \rightarrow \infty}\left(\frac{\left(\beta_{N}^{\mathrm{c}}\right)^{2} \tau_{v}^{-1}}{M^{\mathrm{c}}}\right)^{2} \tau_{e}^{-1}
$$

Then $\lim _{N \rightarrow \infty} M^{\mathrm{c}}=M^{\infty}=\beta_{\infty}^{2} \tau_{v}^{-1}+\sigma_{z_{0}}^{2}$ and $\lim _{N \rightarrow \infty} \beta_{N}^{\mathrm{c}}=\beta_{\infty}=\tau_{e} / \rho$. Thus,

$$
\lim _{N \rightarrow \infty} N \operatorname{var}\left[\tilde{p}_{N}^{\mathrm{c}}-\tilde{p}_{\infty}\right]=\left(\frac{\left(\tau_{e} / \rho\right)^{2} \tau_{v}^{-1}}{\left(\tau_{e} / \rho\right)^{2} \tau_{v}^{-1}+\sigma_{z_{0}}^{2}}\right)^{2} \tau_{e}^{-1}=\frac{\tau_{e}^{3}}{\left(\tau_{e}^{2}+\sigma_{z_{0}}^{2} \rho^{2} \tau_{v}\right)^{2}}
$$

2. It follows from the proof of Proposition 2 that

$$
\lim _{N \rightarrow \infty} N^{2} \operatorname{var}\left[\tilde{p}_{N}-\tilde{p}_{N}^{\mathrm{c}}\right]=\lim _{N \rightarrow \infty}\left(N^{2}\left(a^{2} \tau_{v}^{-1}+b^{2} \sigma_{z_{0}}^{2}\right)\right)
$$

Since $a=\left(M M^{\mathrm{c}}\right)^{-1}\left(\left(\beta_{N}^{2}-\left(\beta_{N}^{\mathrm{c}}\right)^{2}\right) \tau_{v}^{-1} \sigma_{z_{0}}^{2}\right)=\sigma_{z_{0}}^{2}\left(\tau_{v} M M^{\mathrm{c}}\right)^{-1}\left(\beta_{N}+\beta_{N}^{\mathrm{c}}\right)\left(\beta_{N}-\beta_{N}^{\mathrm{c}}\right)$, we have

$$
\lim _{N \rightarrow \infty} N a=\frac{2 \beta_{\infty} \sigma_{z_{0}}^{2}}{\tau_{v}\left(M^{\infty}\right)^{2}} \lim _{N \rightarrow \infty}\left(N\left(\beta_{N}-\beta_{N}^{\mathrm{c}}\right)\right) .
$$

Since $b=\left(\tau_{v} M M^{\mathrm{c}}\right)^{-1}\left(\beta_{N} \beta_{N}^{\mathrm{c}}\left(\beta_{N}^{\mathrm{c}}-\beta_{N}\right)\left(\tau_{v}^{-1}+(1 / N) \tau_{e}^{-1}\right)+\left(\beta_{N}-\beta_{N}^{\mathrm{c}}\right) \sigma_{z_{0}}^{2}\right)$, we have

$$
\lim _{N \rightarrow \infty} N b=\frac{\beta_{\infty}^{2} \tau_{v}^{-1}-\sigma_{z_{0}}^{2}}{\tau_{v}\left(M^{\infty}\right)^{2}} \lim _{N \rightarrow \infty}\left(N\left(\beta_{N}^{\mathrm{c}}-\beta_{N}\right)\right) .
$$

Therefore,

$$
\begin{aligned}
\lim _{N \rightarrow \infty} & N^{2} \operatorname{var}\left[\left(\tilde{p}_{N}-\tilde{p}_{N}^{\mathrm{c}}\right)\right] \\
& =\lim _{N \rightarrow \infty}\left(N^{2}\left(a^{2} \tau_{v}^{-1}+b^{2} \sigma_{z_{0}}^{2}\right)\right) \\
& =\left(\tau_{v}\left(M^{\infty}\right)^{2}\right)^{-2}\left(\left(2 \beta_{\infty} \sigma_{z_{0}}^{2}\right)^{2} \tau_{v}^{-1}+\left(\beta_{\infty}^{2} \tau_{v}^{-1}-\sigma_{z_{0}}^{2}\right)^{2} \sigma_{z_{0}}^{2}\right)\left(\lim _{N \rightarrow \infty}\left(N\left(\beta_{N}^{\mathrm{c}}-\beta_{N}\right)\right)\right)^{2} \\
& =\left(\tau_{v}\left(M^{\infty}\right)^{2}\right)^{-2}\left(\left(\beta_{\infty}^{2} \tau_{v}^{-1}+\sigma_{z_{0}}^{2}\right)^{2} \sigma_{z_{0}}^{2}\right)\left(\lim _{N \rightarrow \infty}\left(N\left(\beta_{N}^{\mathrm{c}}-\beta_{N}\right)\right)\right)^{2} \\
& =\left(\tau_{v} M^{\infty}\right)^{-2} \sigma_{z_{0}}^{2}\left(\lim _{N \rightarrow \infty}\left(N\left(\beta_{N}^{\mathrm{c}}-\beta_{N}\right)\right)\right)^{2}=\frac{\sigma_{z_{0}}^{2} \rho^{4}}{\left(\tau_{e}^{2}+\sigma_{z_{0}}^{2} \rho^{2} \tau_{v}\right)^{2}}\left(\lim _{N \rightarrow \infty}\left(N\left(\beta_{N}^{\mathrm{c}}-\beta_{N}\right)\right)\right)^{2} .
\end{aligned}
$$


Now we have from (5) that

$$
\begin{aligned}
\lim _{N \rightarrow \infty}\left(N\left(\beta_{N}^{\mathrm{c}}-\beta_{N}\right)\right) & =\frac{\tau_{e}}{\rho} \lim _{N \rightarrow \infty}\left(N\left(1-\varphi_{\mathrm{I}}^{\mathrm{c}}\right)-N\left(1-\varphi_{\mathrm{I}}\right) \frac{(1-2 \zeta)}{(1-\zeta)}\right) \\
& =\frac{\tau_{e}}{\rho} \lim _{N \rightarrow \infty}\left(\left(1-\varphi_{\mathrm{I}}\right) \frac{\zeta N}{(1-\zeta)}\right),
\end{aligned}
$$

since by Lemma $1\left(\varphi_{\mathrm{I}}-\varphi_{\mathrm{I}}^{\mathrm{c}}\right) \propto 1 / N^{2}$. Then by $(6)$

$$
\frac{1-\varphi_{\mathrm{I}}}{1-\zeta}=\frac{1}{1-\xi_{\mathrm{I}}} \quad \text { and } \quad \lim _{N \rightarrow \infty} \frac{1}{1-\xi_{\mathrm{I}}}=1
$$

Hence,

$$
\lim _{N \rightarrow \infty}\left(N\left(\beta_{N}^{\mathrm{c}}-\beta_{N}\right)\right)=\frac{\tau_{e}}{\rho} \lim _{N \rightarrow \infty} \zeta N
$$

However, from (6) we have that $\zeta=\xi_{\mathrm{I}}+\varphi_{\mathrm{I}}-\xi_{\mathrm{I}} \varphi_{\mathrm{I}}$. Since $\xi_{\mathrm{I}} \leq \frac{1}{N}$ and $\varphi_{\mathrm{I}} \propto 1 / N$ we get $\lim _{N \rightarrow \infty} \xi_{\mathrm{I}} \varphi_{\mathrm{I}} N=0$. Thus, $\lim _{N \rightarrow \infty} \zeta N=\lim _{N \rightarrow \infty} \xi_{\mathrm{I}} N+\lim _{N \rightarrow \infty} \varphi_{\mathrm{I}} N$. By Lemma 1 $\varphi_{\mathrm{I}}-\varphi_{\mathrm{I}}^{\mathrm{c}} \propto 1 / N^{2}$ and, therefore, $\lim _{N \rightarrow \infty} \varphi_{\mathrm{I}} N=\lim _{N \rightarrow \infty} \varphi_{\mathrm{I}}^{\mathrm{c}} N$. Then from (8) we have

$$
\frac{N^{2} \sigma_{z_{0}}^{2} \rho^{2}}{(N-1) \tau_{e}}=\frac{\left(1-\varphi_{\mathrm{I}}^{\mathrm{c}}\right)^{3}}{\varphi_{\mathrm{I}}^{\mathrm{c}}}
$$

and, thus,

$$
\lim _{N \rightarrow \infty} \varphi_{\mathrm{I}}^{\mathrm{c}} N=\frac{\tau_{e}}{\sigma_{z_{0}}^{2} \rho^{2}}
$$

Hence,

$$
\lim _{N \rightarrow \infty} \zeta N=\lim _{N \rightarrow \infty} \xi_{\mathrm{I}} N+\frac{\tau_{e}}{\sigma_{z_{0}}^{2} \rho^{2}}
$$

As we mentioned above, one conclusion in Theorem 9.2 in Kyle (1989) actually requires a correction. Theorem 9.2 in Kyle (1989) states that $\lim _{N \rightarrow \infty} \xi_{\mathrm{U}} M=0$ which is equivalent to $\lim _{N \rightarrow \infty} \xi_{\mathrm{I}} N=1$. The following lemma corrects that statement.

Lemma 3. We have

$$
\lim _{N \rightarrow \infty} \xi_{\mathrm{I}} N=\frac{\tau_{e}^{2}}{\tau_{v} \sigma_{z_{0}}^{2} \rho^{2}+\tau_{e}^{2}}
$$

and

$$
\lim _{N \rightarrow \infty} \zeta N=\frac{\tau_{e}}{\sigma_{z_{0}}^{2} \rho^{2}}\left(1+\frac{\tau_{e}}{\tau_{v}+\tau_{e}^{2} / \sigma_{z_{0}}^{2} \rho^{2}}\right)=\frac{\tau_{e}}{\sigma_{z_{0}}^{2} \rho^{2}} \frac{\left(\tau_{v}+\tau_{e}\right) \sigma_{z_{0}}^{2} \rho^{2}+\tau_{e}^{2}}{\tau_{v} \sigma_{z_{0}}^{2} \rho^{2}+\tau_{e}^{2}}
$$

Proof. From the formula (7),

$$
\zeta=\varphi_{\mathrm{U}} \frac{\tau_{\mathrm{I}}}{\tau_{\mathrm{U}}}=\varphi_{\mathrm{U}} \frac{\tau_{v}+\tau_{e}+\varphi_{\mathrm{I}}(N-1) \tau_{e}}{\tau_{v}+\varphi_{\mathrm{U}} N \tau_{e}}
$$


Thus,

$$
\lim _{N \rightarrow \infty} \zeta N=\lim _{N \rightarrow \infty}\left(N \varphi_{\mathrm{U}} \frac{\tau_{v}+\tau_{e}+\varphi_{\mathrm{I}}(N-1) \tau_{e}}{\tau_{v}+\varphi_{\mathrm{U}} N \tau_{e}}\right)
$$

and since

$$
\lim _{N \rightarrow \infty} N \varphi_{\mathrm{U}}=\lim _{N \rightarrow \infty} N \varphi_{\mathrm{I}}=\frac{\tau_{e}}{\sigma_{z_{0}}^{2} \rho^{2}}
$$

(see (3)), we obtain

$$
\lim _{N \rightarrow \infty} \zeta N=\frac{\tau_{e}}{\sigma_{z_{0}}^{2} \rho^{2}} \frac{\tau_{v}+\tau_{e}+\left(\tau_{e} / \sigma_{z_{0}}^{2} \rho^{2}\right) \tau_{e}}{\tau_{v}+\left(\tau_{e} / \sigma_{z_{0}}^{2} \rho^{2}\right) \tau_{e}}=\frac{\tau_{e}}{\sigma_{z_{0}}^{2} \rho^{2}}\left(1+\frac{\tau_{e}}{\tau_{v}+\tau_{e}^{2} / \sigma_{z_{0}}^{2} \rho^{2}}\right) .
$$

However, from (6) we have that $\zeta=\xi_{\mathrm{I}}+\varphi_{\mathrm{I}}-\xi_{\mathrm{I}} \varphi_{\mathrm{I}}$. Hence,

$$
\lim _{N \rightarrow \infty} \xi_{\mathrm{I}} N=\lim _{N \rightarrow \infty} \zeta N-\lim _{N \rightarrow \infty} \varphi_{\mathrm{I}} N=\frac{\tau_{e}^{2} / \sigma_{z_{0}}^{2} \rho^{2}}{\tau_{v}+\tau_{e}^{2} / \sigma_{z_{0}}^{2} \rho^{2}}=\frac{\tau_{e}^{2}}{\tau_{v} \sigma_{z_{0}}^{2} \rho^{2}+\tau_{e}^{2}} .
$$

Using Lemma 3, we obtain

$$
\lim _{N \rightarrow \infty}\left(N\left(\beta_{N}^{\mathrm{c}}-\beta_{N}\right)\right)=\frac{\tau_{e}}{\rho} \lim _{N \rightarrow \infty} \zeta N=\frac{\tau_{e}^{2}}{\sigma_{z_{0}}^{2} \rho^{3}} \frac{\left(\tau_{v}+\tau_{e}\right) \sigma_{z_{0}}^{2} \rho^{2}+\tau_{e}^{2}}{\tau_{v} \sigma_{z_{0}}^{2} \rho^{2}+\tau_{e}^{2}} .
$$

Therefore,

$$
\begin{aligned}
\lim _{N \rightarrow \infty} N^{2} \operatorname{var}\left[\tilde{p}_{N}-\tilde{p}_{N}^{\mathrm{c}}\right] & =\frac{\sigma_{z_{0}}^{2} \rho^{4}}{\left(\tau_{e}^{2}+\sigma_{z_{0}}^{2} \rho^{2} \tau_{v}\right)^{2}} \frac{\tau_{e}^{4}}{\sigma_{z_{0}}^{4} \rho^{6}} \frac{\left(\tau_{e}^{2}+\left(\tau_{v}+\tau_{e}\right) \sigma_{z_{0}}^{2} \rho^{2}\right)^{2}}{\left(\tau_{e}^{2}+\sigma_{z_{0}}^{2} \rho^{2} \tau_{v}\right)^{2}} \\
& =\frac{\tau_{e}^{4}}{\sigma_{z_{0}}^{2} \rho^{2}} \frac{\left(\tau_{e}^{2}+\left(\tau_{v}+\tau_{e}\right) \sigma_{z_{0}}^{2} \rho^{2}\right)^{2}}{\left(\tau_{e}^{2}+\tau_{v} \sigma_{z_{0}}^{2} \rho^{2}\right)^{4}}
\end{aligned}
$$

Proof of Proposition 4 In a symmetric linear equilibrium the demands have the form: $\tilde{x}_{N}\left(\tilde{p}, \tilde{\imath}_{n}\right)=\mu_{N}+\beta_{N} \tilde{\imath}_{n}-\gamma_{N} \tilde{p}_{N}, \tilde{x}_{N}^{\mathrm{c}}\left(\tilde{p}, \tilde{\imath}_{n}\right)=\mu_{N}^{\mathrm{c}}+\beta_{N}^{\mathrm{c}} \tilde{\imath}_{n}-\gamma_{N}^{\mathrm{c}} \tilde{p}_{N}^{\mathrm{c}}$ and $\tilde{x}_{\infty}\left(\tilde{p}, \tilde{\imath}_{n}\right)=\mu_{\infty}+\beta_{\infty} \tilde{\imath}_{n}-$ $\gamma_{\infty} \tilde{p}_{\infty}$. Kyle (1989, proofs of Theorems 5.1 and 6.1) shows that $\mu_{N}^{\mathrm{c}}=\mu_{N}=0$. Hence, $\mu_{\infty}=0$ as well. Recall that we showed in Lemma 2 that $\left(\beta_{N}^{\mathrm{c}}-\beta_{\infty}\right) \propto\left(\beta_{N}-\beta_{\infty}\right) \propto\left(\beta_{N}-\beta_{N}^{\mathrm{c}}\right) \propto 1 / N$. Now let us prove an analogous result for $\gamma_{N}, \gamma_{N}^{\mathrm{c}}$ and $\gamma_{\infty}$.

Lemma 4. We have $\gamma_{\infty} \neq 0, \gamma_{N}^{\mathrm{c}}-\gamma_{\infty}=O(1 / N), \gamma_{N}-\gamma_{\infty}=O(1 / N)$ and $\gamma_{N}-\gamma_{N}^{\mathrm{c}}=O(1 / N)$.

Proof. Kyle (1989, expressions (B.4) and (C.5)) provides the following formulas for $\gamma_{N}^{\mathrm{c}}$ and $\gamma_{N}:$

$$
\gamma_{N}^{\mathrm{c}}=\frac{\tau_{\mathrm{I}}}{\rho}-\frac{\varphi_{\mathrm{I}} \tau_{e}}{\lambda \beta_{N} \rho} \quad \text { and } \quad \gamma_{N}=\frac{\lambda \beta_{N} \tau_{\mathrm{I}}-\varphi_{\mathrm{I}} \tau_{e}}{\lambda \beta_{N}\left(\lambda_{\mathrm{I}} \tau_{\mathrm{I}}+\rho\right)} \quad \text { where } \frac{\lambda}{\lambda_{\mathrm{I}}}=1-\xi_{\mathrm{I}}
$$

Corollary 4.1 in Kyle (1989) implies that in our case with the competitive market makers 
sector we have $\varphi_{\mathrm{U}} \tau_{e}=\lambda \beta_{N} \tau_{\mathrm{U}}$. Thus, using (3) we obtain that

$$
\begin{aligned}
& \gamma_{N}^{\mathrm{c}}=\frac{\tau_{\mathrm{I}}}{\rho}-\frac{\varphi_{\mathrm{I}} \tau_{\mathrm{U}}}{\varphi_{\mathrm{U}} \rho}=\frac{\tau_{\mathrm{I}}}{\rho}-\frac{\tau_{\mathrm{U}}}{\rho}\left(1-\frac{1}{N}\left(1-\varphi_{\mathrm{I}}\right)\right) \text { and that } \\
& \gamma_{N}=\frac{\tau_{\mathrm{I}}}{\lambda_{\mathrm{I}} \tau_{\mathrm{I}}+\rho}-\frac{\varphi_{\mathrm{I}} \tau_{\mathrm{U}}}{\varphi_{\mathrm{U}}\left(\lambda_{\mathrm{I}} \tau_{\mathrm{I}}+\rho\right)}=\frac{\tau_{\mathrm{I}}}{\lambda_{\mathrm{I}} \tau_{\mathrm{I}}+\rho}-\frac{\tau_{\mathrm{U}}}{\left(\lambda_{\mathrm{I}} \tau_{\mathrm{I}}+\rho\right)}\left(1-\frac{1}{N}\left(1-\varphi_{\mathrm{I}}\right)\right) .
\end{aligned}
$$

Then since $\left(\tau_{\mathrm{I}}\right)_{N}=\left(\tau_{\mathrm{I}}\right)_{\infty}+O(1 / N),\left(\tau_{\mathrm{U}}\right)_{N}=\left(\tau_{\mathrm{U}}\right)_{\infty}+O(1 / N), \varphi_{\mathrm{I}}=O(1 / N)$, and $\lambda_{\mathrm{I}}=$ $\lambda /\left(1-\xi_{\mathrm{I}}\right)=O(\lambda)+O(1 / N)=O\left(\varphi_{\mathrm{U}}\right)+O(1 / N)=O(1 / N)$, we obtain that $\gamma_{\infty} \neq 0$, $\gamma_{N}^{\mathrm{c}}-\gamma_{\infty}=O(1 / N)$ and $\gamma_{N}-\gamma_{\infty}=O(1 / N)$. Then, also, $\gamma_{N}-\gamma_{N}^{\mathrm{c}}=O(1 / N)$.

Now for simplicity of notation let us rewrite the prices expressions (9) and (10) in the following way:

$$
\begin{aligned}
& \tilde{p}_{N}^{\mathrm{c}}=A_{N}^{\mathrm{c}} \tilde{v}+B_{N}^{\mathrm{c}} \frac{1}{N} \sum_{n=1}^{N} \tilde{e}_{n}+C_{N}^{\mathrm{c}} \tilde{z}_{0}, \\
& \tilde{p}_{N}=A_{N} \tilde{v}+B_{N} \frac{1}{N} \sum_{n=1}^{N} \tilde{e}_{n}+C_{N} \tilde{z}_{0}, \\
& \tilde{p}_{\infty}=A_{\infty} \tilde{v}+C_{\infty} \tilde{z}_{0} .
\end{aligned}
$$

It follows from the proof of Proposition 1 that $A_{N}^{\mathrm{c}}$ and $A_{N}$ converge to $A_{\infty}, B_{N}^{\mathrm{c}}$ and $B_{N}$ converge to $B_{\infty} \neq 0$ and $C_{N}^{\mathrm{c}}$ and $C_{N}$ converge to $C_{\infty}$. All of these convergencies also happen at a rate of $1 / N$ or faster. Then

$\tilde{x}_{N}^{\mathrm{c}}\left(\tilde{p}, \tilde{\imath}_{n}\right)=\beta_{N}^{\mathrm{c}} \tilde{\imath}_{n}-\gamma_{N}^{\mathrm{c}} \tilde{p}_{N}^{\mathrm{c}}=\left(\beta_{N}^{\mathrm{c}}-\gamma_{N}^{\mathrm{c}} A_{N}^{\mathrm{c}}\right) \tilde{v}+\left(\beta_{N}^{\mathrm{c}}-\gamma_{N}^{\mathrm{c}} B_{N}^{\mathrm{c}} \frac{1}{N}\right) \tilde{e}_{n}-\gamma_{N}^{\mathrm{c}} B_{N}^{\mathrm{c}} \frac{1}{N} \sum_{k \neq n} \tilde{e}_{k}-\gamma_{N}^{\mathrm{c}} C_{N}^{\mathrm{c}} \tilde{z}_{0}$

A similar expression can be written for $\tilde{x}_{N}\left(\tilde{p}, \tilde{\imath}_{n}\right)$. Now let us define $D_{a}=\left(\beta_{N}^{\mathrm{c}}-\gamma_{N}^{\mathrm{c}} A_{N}^{\mathrm{c}}\right)-$ $\left(\beta_{N}-\gamma_{N} A_{N}\right), D_{b}=\left(\beta_{N}^{\mathrm{c}}-\gamma_{N}^{\mathrm{c}} B_{N}^{\mathrm{c}}(1 / N)\right)-\left(\beta_{N}-\gamma_{N} B_{N}(1 / N)\right), D_{\mathrm{c}}=\gamma_{N}^{\mathrm{c}} B_{N}^{\mathrm{c}}-\gamma_{N} B_{N}$ and $D_{d}=\gamma_{N}^{\mathrm{c}} C_{N}^{\mathrm{c}}-\gamma_{N} C_{N}$. Then we obtain

$$
E\left[\left(\tilde{x}_{N}^{\mathrm{c}}\left(\tilde{p}, \tilde{\imath}_{n}\right)-\tilde{x}_{N}\left(\tilde{p}, \tilde{\imath}_{n}\right)\right)^{2}\right]=D_{a}^{2} \tau_{v}^{-1}+D_{b}^{2} \tau_{e}^{-1}+D_{\mathrm{c}}^{2} \frac{N-1}{N^{2}} \tau_{v}^{-1}+D_{d}^{2} \sigma_{z_{0}}^{2} .
$$

It is immediate that $D_{a}=O(1 / N), D_{\mathrm{c}}=O(1 / N), D_{d}=O(1 / N)$, while

$$
D_{b}=\left(\beta_{N}^{\mathrm{c}}-\beta_{N}\right)-\frac{1}{N}\left(\gamma_{N}^{\mathrm{c}} B_{N}^{\mathrm{c}}-\gamma_{N} B_{N}\right) \propto \frac{1}{N}-\frac{1}{N} O(1 / N) \propto 1 / N .
$$

Therefore,

$$
E\left[\left(\tilde{x}_{N}^{\mathrm{c}}\left(\tilde{p}, \tilde{\imath}_{n}\right)-\tilde{x}_{N}\left(\tilde{p}, \tilde{\imath}_{n}\right)\right)^{2}\right] \propto 1 / N^{2}
$$


Now let us note that

$$
\tilde{x}_{\infty}\left(\tilde{p}, \tilde{\imath}_{n}\right)=\beta_{\infty} \tilde{\imath}_{n}-\gamma_{\infty} \tilde{p}_{\infty}=\left(\beta_{\infty}-\gamma_{\infty} A_{\infty}\right) \tilde{v}+\beta_{\infty} \tilde{e}_{n}-\gamma_{\infty} C_{\infty} \tilde{z}_{0}
$$

So if we define $K_{a}=\left(\beta_{N}^{\mathrm{c}}-\gamma_{N}^{\mathrm{c}} A_{N}^{\mathrm{c}}\right)-\left(\beta_{\infty}-\gamma_{\infty} A_{\infty}\right), K_{b}=\left(\beta_{N}^{\mathrm{c}}-\gamma_{N}^{\mathrm{c}} B_{N}^{\mathrm{c}}(1 / N)\right)-\beta_{\infty}$, $K_{\mathrm{c}}=\gamma_{N}^{\mathrm{c}} B_{N}^{\mathrm{c}}$ and $K_{d}=\gamma_{N}^{\mathrm{c}} C_{N}^{\mathrm{c}}-\gamma_{\infty} C_{\infty}$, then we obtain

$$
E\left[\left(\tilde{x}_{N}^{\mathrm{c}}\left(\tilde{p}, \tilde{\imath}_{n}\right)-\tilde{x}_{\infty}\left(\tilde{p}, \tilde{\imath}_{n}\right)\right)^{2}\right]=K_{a}^{2} \tau_{v}^{-1}+K_{b}^{2} \tau_{e}^{-1}+K_{\mathrm{c}}^{2} \frac{N-1}{N^{2}} \tau_{v}^{-1}+K_{d}^{2} \sigma_{z_{0}}^{2} .
$$

It is immediate that $K_{a}=O(1 / N), K_{b}=O(1 / N), K_{d}=O(1 / N)$, while $K_{\mathrm{c}}=\gamma_{N}^{\mathrm{c}} B_{N}^{\mathrm{c}}$ converges to $\gamma_{\infty} B_{\infty} \neq 0$. Hence,

$$
E\left[\left(\tilde{x}_{N}^{\mathrm{c}}\left(\tilde{p}, \tilde{\imath}_{n}\right)-\tilde{x}_{\infty}\left(\tilde{p}, \tilde{\imath}_{n}\right)\right)^{2}\right] \propto 1 / N
$$

Proof of Proposition 5 First for simplicity of notation let us again rewrite the expression for prices (9) as in the proof of Proposition 4:

$$
\begin{aligned}
& \tilde{p}_{N}^{\mathrm{c}}=A_{N}^{\mathrm{c}} \tilde{v}+B_{N}^{\mathrm{c}} \frac{1}{N} \sum_{k=1}^{N} \tilde{e}_{k}+C_{N}^{\mathrm{c}} \tilde{z}_{0}, \\
& \tilde{p}_{N}=A_{N} \tilde{v}+B_{N} \frac{1}{N} \sum_{k=1}^{N} \tilde{e}_{k}+C_{N} \tilde{z}_{0} .
\end{aligned}
$$

Then, also as in the proof of Proposition 4, let us obtain an expression for $\tilde{x}_{N}^{\mathrm{c}}\left(\tilde{p}, \tilde{\imath}_{n}\right)$ :

$$
\tilde{x}_{N}^{\mathrm{c}}\left(\tilde{p}, \tilde{\imath}_{n}\right)=\left(\beta_{N}^{\mathrm{c}}-\gamma_{N}^{\mathrm{c}} A_{N}^{\mathrm{c}}\right) \tilde{v}+\left(\beta_{N}^{\mathrm{c}}-\gamma_{N}^{\mathrm{c}} B_{N}^{\mathrm{c}} \frac{1}{N}\right) \tilde{e}_{n}-\gamma_{N}^{\mathrm{c}} B_{N}^{\mathrm{c}} \frac{1}{N} \sum_{k \neq n} \tilde{e}_{k}-\gamma_{N}^{\mathrm{c}} C_{N}^{\mathrm{c}} \tilde{z}_{0}
$$

Note that the expression for $\tilde{x}_{N}\left(\tilde{p}, \tilde{\imath}_{n}\right)$ is just similar (with no superscripts of $c$ ). That allows us to obtain an explicit expression for $\tilde{\pi}_{n}^{\mathrm{c}}\left(\tilde{p}, \tilde{\imath}_{n}\right)=\left(\tilde{v}-\tilde{p}_{N}^{\mathrm{c}}\right) \tilde{x}_{N}^{\mathrm{c}}\left(\tilde{p}, \tilde{\imath}_{n}\right)$ as a function of $\tilde{v}, \tilde{e}_{1}, \ldots, \tilde{e}_{N}, \tilde{z}_{0}$. Note that the expression for $\tilde{\pi}_{n}$ is again different only in the absence of the superscript c. Actually the difference between any corresponding coefficients in $\tilde{\pi}_{n}$ and $\tilde{\pi}_{n}^{\mathrm{c}}$ will be of the order $O(1 / N)$. So all of the coefficients in $\left(\tilde{\pi}_{n}-\tilde{\pi}_{n}^{\mathrm{c}}\right)$ will be of the order of $O(1 / N)$. Moreover $\left(\tilde{\pi}_{n}-\tilde{\pi}_{n}^{\mathrm{c}}\right)$ will be a homogenous polynomial of the second degree. Hence, we obtain that $\left(\tilde{\pi}_{N}-\tilde{\pi}_{N}^{\mathrm{c}}\right)^{2}$ is a homogenous polynomial of the forth degree. Since $\tilde{v}, \tilde{e}_{1}, \ldots, \tilde{e}_{N}, \tilde{z}_{0}$ are all independent with zero expectations, in $E\left[\left(\tilde{\pi}_{N}-\tilde{\pi}_{N}^{\mathrm{c}}\right)^{2}\right]$ only all of the fourths moments and all products of the second moments of $\tilde{v}, \tilde{e}_{1}, \ldots, \tilde{e}_{N}, \tilde{z}_{0}$ will be nonzero. It is easy to notice that all of the coefficients in this expression will be of the order of $O(1 / N) O(1 / N)=O\left(1 / N^{2}\right)$. Therefore,

$$
E\left[\left(\tilde{\pi}_{N}-\tilde{\pi}_{N}^{\mathrm{c}}\right)^{2}\right]=O\left(1 / N^{2}\right)
$$


To show that the rate of convergence is exactly $1 / N^{2}$ we need to be much more specific when describing $E\left[\left(\tilde{\pi}_{N}-\tilde{\pi}_{N}^{\mathrm{c}}\right)^{2}\right]$. For some coefficients $L_{v}, L_{z}, L_{v z}, L_{v n}, L_{k n}, L_{n z}$, where $1 \leq k, m \leq N$, we can write

$$
\begin{aligned}
\tilde{\pi}_{N}\left(\tilde{p}, \tilde{\imath}_{n}\right)-\tilde{\pi}_{N}^{\mathrm{c}}\left(\tilde{p}, \tilde{\imath}_{n}\right)= & \left(L_{v} \tilde{v}^{2}+\sum_{k=1}^{N} L_{k k} \tilde{e}_{k}^{2}+L_{z} \tilde{z}_{0}^{2}\right) \\
& +\left(\sum_{k=1}^{N} L_{v k} \tilde{v} \tilde{e}_{k}+L_{v z} \tilde{v} \tilde{z}_{0}+\sum_{k \neq m} L_{k m} \tilde{e}_{k} \tilde{e}_{m}+\sum_{k=1}^{N} L_{k z} \tilde{e}_{k} \tilde{z}_{0}\right) .
\end{aligned}
$$

Note that all of the coefficients $L_{v}, L_{z}, L_{v z}, L_{v n}, L_{k m}, L_{k z}$, where $1 \leq k, m \leq N$ are of the order $O(1 / N)$. Since only the fourths moments and all products of the second moments of $\tilde{v}, \tilde{e}_{1}, \ldots, \tilde{e}_{N}, \tilde{z}_{0}$ will be nonzero in $E\left[\left(\tilde{\pi}_{N}-\tilde{\pi}_{N}^{\mathrm{c}}\right)^{2}\right]$, we obtain that

$$
\begin{aligned}
E\left[\left(\tilde{\pi}_{N}\left(\tilde{p}, \tilde{\imath}_{n}\right)-\tilde{\pi}_{N}^{\mathrm{c}}\left(\tilde{p}, \tilde{\imath}_{n}\right)\right)^{2}\right]= & E\left[\left(L_{v} \tilde{v}^{2}+\sum_{k=1}^{N} L_{k k} \tilde{e}_{n}^{2}+L_{z} \tilde{z}_{0}^{2}\right)^{2}\right] \\
& +\sum_{k=1}^{N} L_{v k}^{2} \tau_{v}^{-1} \tau_{e}^{-1}+L_{v z}^{2} \tau_{v}^{-1} \sigma_{z_{0}}^{2}+\sum_{k \neq m} L_{k m}^{2} \tau_{e}^{-1} \tau_{e}^{-1}+\sum_{k=1}^{N} L_{k z}^{2} \tau_{e}^{-1} \sigma_{z_{0}}^{2} .
\end{aligned}
$$

Since all parts of this expression are positive and of the order of $O\left(1 / N^{2}\right)$, it is enough to demonstrate that one part is actually of the order of $1 / N^{2}$ to conclude that the entire expression is of the order of $1 / N^{2}$. Let us examine $L_{k z}$ for $k=n$ more closely. One obtains that

$$
\begin{gathered}
=\left(-C_{N}\left(\beta_{N}-\gamma_{N} B_{N} \frac{1}{N}\right)+B_{N} \frac{1}{N}\left(-\gamma_{N} C_{N}\right)\right) \\
-\left(-C_{N}^{\mathrm{c}}\left(\beta_{N}^{\mathrm{c}}-\gamma_{N}^{\mathrm{c}} B_{N}^{\mathrm{c}} \frac{1}{N}\right)+B_{N}^{\mathrm{c}} \frac{1}{N}\left(-\gamma_{N}^{\mathrm{c}} C_{N}^{\mathrm{c}}\right)\right) \\
=-\left(C_{N} \beta_{N}-C_{N}^{\mathrm{c}} \beta_{N}^{\mathrm{c}}\right)+\frac{1}{N}\left(\left(\gamma_{N} B_{N}-\gamma_{N}^{\mathrm{c}} B_{N}^{\mathrm{c}}\right)\right)-\frac{1}{N}\left(B_{N} \gamma_{N} C_{N}-B_{N}^{\mathrm{c}} \gamma_{N}^{\mathrm{c}} C_{N}^{\mathrm{c}}\right) \\
=-\left(C_{N} \beta_{N}-C_{N}^{\mathrm{c}} \beta_{N}^{\mathrm{c}}\right)+\frac{1}{N} O(1 / N)+\frac{1}{N} O(1 / N) \\
=-\left(C_{N} \beta_{N}-C_{N}^{\mathrm{c}} \beta_{N}^{\mathrm{c}}\right)+O\left(1 / N^{2}\right) .
\end{gathered}
$$

Inspection of (9) shows that $C_{N} \beta_{N}=A_{N}$ and $C_{N}^{\mathrm{c}} \beta_{N}^{\mathrm{c}}=A_{N}^{\mathrm{c}}$, while in the proof of Proposition 1 we demonstrated that $\left(A_{N}-A_{N}^{\mathrm{c}}\right)=a=\left(M M^{\mathrm{c}}\right)^{-1}\left(\left(\beta_{N}^{2}-\left(\beta_{N}^{\mathrm{c}}\right)^{2}\right) \tau_{v}^{-1} \sigma_{z_{0}}^{2}\right) \propto\left(\beta_{N}-\beta_{N}^{\mathrm{c}}\right) \propto$ $1 / N$. Therefore, $L_{n z} \propto 1 / N$. Hence $L_{n z}^{2} \propto 1 / N^{2}$ and

$$
E\left[\left(\tilde{\pi}_{N}\left(\tilde{p}, \tilde{\imath}_{n}\right)-\tilde{\pi}_{N}^{\mathrm{c}}\left(\tilde{p}, \tilde{\imath}_{n}\right)\right)^{2}\right] \propto L_{n z}^{2} \propto 1 / N^{2} .
$$

This finishes the proof for the strategic effect. Now let us examine the limit effect. The proof 
is more straightforward. Recall from the proof of Proposition 2 that

$$
\begin{aligned}
\tilde{x}_{\infty}\left(\tilde{p}, \tilde{l}_{n}\right) & =\left(\beta_{\infty}-\gamma_{\infty} A_{\infty}\right) \tilde{v}+\beta_{\infty} \tilde{e}_{n}-\gamma_{\infty} C_{\infty} \tilde{z}_{0} \quad \text { and } \\
\left(\tilde{v}-\tilde{p}_{\infty}\right) & =\left(1-A_{\infty}\right) \tilde{v}-C_{\infty} \tilde{z}_{0}
\end{aligned}
$$

while

$$
\begin{aligned}
& \tilde{x}_{N}^{\mathrm{c}}\left(\tilde{p}, \tilde{\imath}_{n}\right)=\left(\beta_{N}^{\mathrm{c}}-\gamma_{N}^{\mathrm{c}} A_{N}^{\mathrm{c}}\right) \tilde{v}+\left(\beta_{N}^{\mathrm{c}}-\gamma_{N}^{\mathrm{c}} B_{N}^{\mathrm{c}} \frac{1}{N}\right) \tilde{e}_{n}-\gamma_{N}^{\mathrm{c}} B_{N}^{\mathrm{c}} \frac{1}{N} \sum_{k \neq n} \tilde{e}_{k}-\gamma_{N}^{\mathrm{c}} C_{N}^{\mathrm{c}} \tilde{z}_{0} \text { and } \\
& \left(\tilde{v}-\tilde{p}_{N}^{\mathrm{c}}\right)=\left(1-A_{N}^{\mathrm{c}}\right) \tilde{v}-B_{N}^{\mathrm{c}} \frac{1}{N} \tilde{e}_{n}-B_{N}^{\mathrm{c}} \frac{1}{N} \sum_{k \neq n} \tilde{e}_{k}-C_{N}^{\mathrm{c}} \tilde{z}_{0} .
\end{aligned}
$$

By the same argument as the above for the strategic effect, in $E\left[\left(\tilde{\pi}_{n}^{\mathrm{c}}-\tilde{\pi}_{\infty}\right)^{2}\right]$ only all of the fourth moments and all products of the second moments of $\tilde{v}, \tilde{e}_{1}, \ldots, \tilde{e}_{N}, \tilde{z}_{0}$ will be nonzero. Similarly to the case of strategic effect, all coefficients will converge to zero at a rate of $O\left(1 / N^{2}\right)$ except for the parts where some moments of $\tilde{e}_{k}, k \neq n$ are included. (Note that for the rates of convergence we can ignore parts associated with $\tilde{e}_{n}$ and not some other $\tilde{e}_{k}$, $k \neq n$.) Hence the only parts of $E\left[\left(\tilde{\pi}_{n}^{\mathrm{c}}-\tilde{\pi}_{\infty}\right)^{2}\right]$ that have to be examined closely involve moments of $\tilde{e}_{k}, k \neq n$. So we have to concentrate on the following remaining difference between $\tilde{\pi}_{n}^{\mathrm{c}}$ and $\tilde{\pi}_{\infty}$ :

$$
\left(B_{N}^{\mathrm{c}} \frac{1}{N} \sum_{k \neq n} \tilde{e}_{k}\right) \tilde{x}_{N}^{\mathrm{c}}\left(\tilde{p}, \tilde{\imath}_{n}\right)+\left(\gamma_{N}^{\mathrm{c}} B_{N}^{\mathrm{c}} \frac{1}{N} \sum_{k \neq n} \tilde{e}_{k}\right)\left(\tilde{v}-\tilde{p}_{N}^{\mathrm{c}}\right) .
$$

Rewriting this expression, we obtain

$$
B_{N}^{\mathrm{c}}\left(\frac{1}{N} \sum_{k \neq n} \tilde{e}_{k}\right)\left(\tilde{x}_{N}^{\mathrm{c}}\left(\tilde{p}, \tilde{\imath}_{n}\right)+\gamma_{N}^{\mathrm{c}}\left(\tilde{v}-\tilde{p}_{N}^{\mathrm{c}}\right)\right) .
$$

Substituting expressions for $\tilde{x}_{N}^{\mathrm{c}}\left(\tilde{p}, \tilde{\imath}_{n}\right)$ and $\left(\tilde{v}-\tilde{p}_{N}^{\mathrm{c}}\right)$ let us exclude parts that will be $O\left(1 / N^{2}\right)$ once $E\left[\left(\tilde{\pi}_{n}^{\mathrm{c}}-\tilde{\pi}_{\infty}\right)^{2}\right]$ is computed. Then we obtain the following remaining difference between $\tilde{\pi}_{n}^{\mathrm{c}}$ and $\tilde{\pi}_{\infty}$ :

$$
\begin{aligned}
& B_{N}^{\mathrm{c}} \frac{1}{N} \sum_{k \neq n} \tilde{e}_{k}\left(\left(\beta_{N}^{\mathrm{c}}-\gamma_{N}^{\mathrm{c}} A_{N}^{\mathrm{c}}\right) \tilde{v}+\left(\beta_{N}^{\mathrm{c}}-\gamma_{N}^{\mathrm{c}} B_{N}^{\mathrm{c}} \frac{1}{N}\right) \tilde{e}_{n}-\gamma_{N}^{\mathrm{c}} C_{N}^{\mathrm{c}} \tilde{z}_{0}+\gamma_{N}^{\mathrm{c}}\left(1-A_{N}^{\mathrm{c}}\right) \tilde{v}-\gamma_{N}^{\mathrm{c}} C_{N}^{\mathrm{c}} \tilde{z}_{0}\right) \\
& =B_{N}^{\mathrm{c}} \frac{1}{N} \sum_{k \neq n} \tilde{e}_{k}\left(\left(\beta_{N}^{\mathrm{c}}+\gamma_{N}^{\mathrm{c}}-2 \gamma_{N}^{\mathrm{c}} A_{N}^{\mathrm{c}}\right) \tilde{v}+\left(\beta_{N}^{\mathrm{c}}-\gamma_{N}^{\mathrm{c}} B_{N}^{\mathrm{c}} \frac{1}{N}\right) \tilde{e}_{n}-2 \gamma_{N}^{\mathrm{c}} C_{N}^{\mathrm{c}} \tilde{z}_{0}\right) .
\end{aligned}
$$


Therefore,

$$
\begin{aligned}
& E\left[\left(\tilde{\pi}_{n}^{\mathrm{c}}\left(\tilde{p}, \tilde{\imath}_{n}\right)-\tilde{\pi}_{\infty}\left(\tilde{p}, \tilde{\imath}_{n}\right)\right)^{2}\right]=O\left(1 / N^{2}\right) \\
& \quad+\left(B_{N}^{\mathrm{c}}\right)^{2} \frac{N-1}{N^{2}} \tau_{v}^{-1}\left(\left(\beta_{N}^{\mathrm{c}}+\gamma_{N}^{\mathrm{c}}-2 \gamma_{N}^{\mathrm{c}} A_{N}^{\mathrm{c}}\right)^{2} \tau_{v}^{-1}+\left(\beta_{N}^{\mathrm{c}}-\gamma_{N}^{\mathrm{c}} B_{N}^{\mathrm{c}} \frac{1}{N}\right)^{2} \tau_{v}^{-1}+\left(2 \gamma_{N}^{\mathrm{c}} C_{N}^{\mathrm{c}}\right)^{2} \sigma_{z_{0}}^{2}\right) .
\end{aligned}
$$

Since $B_{\infty} \neq 0, C_{\infty} \neq 0, \beta_{\infty} \neq 0$ and $\gamma_{\infty} \neq 0$, it is immediate that both of the last two parts are nonzero, hence

$$
E\left[\left(\tilde{\pi}_{n}^{\mathrm{c}}\left(\tilde{p}, \tilde{\imath}_{n}\right)-\tilde{\pi}_{\infty}\left(\tilde{p}, \tilde{\imath}_{n}\right)\right)^{2}\right] \propto 1 / N .
$$

Proof of Proposition 6 First, note that using the full Taylor expansion for the exponential function we obtain, for two profit levels $\pi_{a}$ and $\pi_{b}$, that

$$
U\left(\pi_{a}\right) / U\left(\pi_{b}\right)-1=\exp \left(\rho\left(\pi_{b}-\pi_{a}\right)\right)-1=\sum_{k=1}^{\infty} \frac{1}{k !} \rho^{k}\left(\pi_{b}-\pi_{a}\right)^{k}
$$

and, therefore,

$$
\left(U\left(\pi_{a}\right) / U\left(\pi_{b}\right)-1\right)^{2}=\sum_{k=2}^{\infty} \Lambda_{k} \rho^{k}\left(\pi_{b}-\pi_{a}\right)^{k}
$$

with coefficients

$$
\Lambda_{k}=\sum_{l=1}^{k-1} \frac{1}{l !} \frac{1}{(k-l) !}
$$

Hence, we obtain

$$
E\left[\left(U\left(\pi_{a}\right) / U\left(\pi_{b}\right)-1\right)^{2}\right]=\sum_{k=2}^{\infty} \Lambda_{k} \rho^{k} E\left[\left(\pi_{b}-\pi_{a}\right)^{k}\right]
$$

Now for the strategic effect the argument is straightforward. Recall from the proof of Proposition 5 that all of the coefficients in $\left(\tilde{\pi}_{n}-\tilde{\pi}_{n}^{\mathrm{c}}\right)$ will be of the order of $O(1 / N)$. Therefore, all of the coefficients in $E\left[\left(\tilde{\pi}_{N}-\tilde{\pi}_{N}^{\mathrm{c}}\right)^{k}\right]$ will be of the order of $O\left(1 / N^{k}\right)$, while we have proved in Proposition 5 that $E\left[\left(\tilde{\pi}_{N}-\tilde{\pi}_{N}^{\mathrm{c}}\right)^{2}\right] \propto 1 / N^{2}$. Thus,

$$
E\left[\left(U\left(\tilde{\pi}_{N}\right) / U\left(\tilde{\pi}_{N}^{\mathrm{c}}\right)-1\right)^{2}\right] \propto E\left[\left(U\left(\tilde{\pi}_{N}^{\mathrm{c}}\right) / U\left(\tilde{\pi}_{N}\right)-1\right)^{2}\right] \propto 1 / N^{2} .
$$

For the limit effect the argument is similar. Recall that in the proof of Proposition 5 we have showed that all of the coefficients in $\left(\tilde{\pi}_{n}^{\mathrm{c}}-\tilde{\pi}_{\infty}\right)$ are similar to those in $\left(\tilde{\pi}_{n}-\tilde{\pi}_{n}^{\mathrm{c}}\right)$ with the exception of those related to $\tilde{e}_{k}, k \neq n$. The straightforward inspection of this difference reveals that nevertheless $\sum_{k=3}^{\infty} \Lambda_{k} \rho^{k} E\left[\left(\pi_{b}-\pi_{a}\right)^{k}\right]$ will be of the order $O\left(1 / N^{2}\right)$, while we have proved in Proposition 5 that $E\left[\left(\tilde{\pi}_{n}^{\mathrm{c}}-\tilde{\pi}_{\infty}\right)^{2}\right] \propto 1 / N$. Thus,

$$
E\left[\left(U\left(\tilde{\pi}_{N}^{\mathrm{c}}\right) / U\left(\tilde{\pi}_{\infty}\right)-1\right)^{2}\right] \propto E\left[\left(U\left(\tilde{\pi}_{\infty}\right) / U\left(\tilde{\pi}_{N}^{\mathrm{c}}\right)-1\right)^{2}\right] \propto 1 / N
$$


Finally, for $E\left[\left(U\left(\tilde{\pi}_{N}\right) / U\left(\tilde{\pi}_{\infty}\right)-1\right)^{2}\right]$ the argument is exactly the same as it is for $E\left[\left(U\left(\tilde{\pi}_{N}^{\mathrm{c}}\right) / U\left(\tilde{\pi}_{\infty}\right)-\right.\right.$ $\left.1)^{2}\right]$.

Proof of Proposition 7 Note first, that the parts 2 and 3 of Proposition 7 and the fact that $\varphi_{\mathrm{I}}^{\mathrm{c}}, \varphi_{\mathrm{I}}, \varphi_{\mathrm{U}}^{\mathrm{c}}$, and $\varphi_{\mathrm{U}}$ converge to zero at the rate $N / \sigma_{z}^{2}(N)$ were proved in the proof of Proposition 1 . Thus, we only need to prove part 1 . The proof proceeds in the similar way to the proof of Proposition 2. Since $(1-\zeta)=\left(1-\xi_{\mathrm{I}}\right)\left(1-\varphi_{\mathrm{I}}\right)$ and $0 \leq \xi_{\mathrm{I}} \leq 1 / N$ (see (6)), we have $\zeta_{N} \propto N / \sigma_{z}^{2}(N)$. Then as in the proof of Lemma 1:

$$
\frac{\left(1-\varphi_{\mathrm{I}}\right)^{3}}{\left(1-\varphi_{\mathrm{I}}^{\mathrm{c}}\right)^{3}} \frac{\varphi_{\mathrm{I}}^{\mathrm{c}}}{\varphi_{\mathrm{I}}}-1=\frac{\zeta(2-3 \zeta)}{(1-\zeta)^{2}} \propto \frac{N}{\sigma_{z}^{2}(N)}
$$

and

$$
\begin{aligned}
\frac{\left(1-\varphi_{\mathrm{I}}\right)^{3}}{\left(1-\varphi_{\mathrm{I}}^{\mathrm{c}}\right)^{3}} \frac{\varphi_{\mathrm{I}}^{\mathrm{c}}}{\varphi_{\mathrm{I}}}-1 & =\frac{\left(1-\varphi_{\mathrm{I}}\right)^{3}}{\left(1-\varphi_{\mathrm{I}}^{\mathrm{c}}\right)^{3}}\left(\frac{\varphi_{\mathrm{I}}^{\mathrm{c}}}{\varphi_{\mathrm{I}}}-1\right)+\left(\frac{\left(1-\varphi_{\mathrm{I}}\right)^{3}}{\left(1-\varphi_{\mathrm{I}}^{\mathrm{c}}\right)^{3}}-1\right) \\
& \propto \varphi_{\mathrm{I}}^{-1}\left(\varphi_{\mathrm{I}}-\varphi_{\mathrm{I}}^{\mathrm{c}}\right)+O\left(\varphi_{\mathrm{I}}-\varphi_{\mathrm{I}}^{\mathrm{c}}\right) \propto \frac{\sigma_{z}^{2}(N)}{N}\left(\varphi_{\mathrm{I}}-\varphi_{\mathrm{I}}^{\mathrm{c}}\right)
\end{aligned}
$$

Hence, $\left(\varphi_{\mathrm{I}}\right)_{N}-\left(\varphi_{\mathrm{I}}^{\mathrm{c}}\right)_{N} \propto N^{2} / \sigma_{z}^{4}(N)$. Then $\beta_{N}$ and $\beta_{N}^{\mathrm{c}}$ converge to $\beta_{\infty}=\tau_{e} / \rho$ and from (2) we obtain

$$
\varphi_{\mathrm{I}}-\varphi_{\mathrm{I}}^{\mathrm{c}}=\frac{(N-1) \sigma_{z}^{2} \tau_{e}\left(\beta_{N}^{2}-\left(\beta_{N}^{\mathrm{c}}\right)^{2}\right)}{\left((N-1) \beta_{N}^{2}+\sigma_{z}^{2} \tau_{e}\right)\left((N-1)\left(\beta_{N}^{\mathrm{c}}\right)^{2}+\sigma_{z}^{2} \tau_{e}\right)} \propto \frac{N \sigma_{z}^{2}(N)\left(\beta_{N}-\beta_{N}^{\mathrm{c}}\right)}{\sigma_{z}^{4}(N)} .
$$

Thus, $\beta_{N}-\beta_{N}^{\mathrm{c}} \propto N / \sigma_{z}^{2}(N)$. Now let $\tilde{z}_{0}:=\tilde{z}(N) / N$. Then $\sigma_{z_{0}}^{2}:=\operatorname{var}\left[\tilde{z}_{0}\right]=\sigma_{z}^{2}(N) / N^{2}$. Now let us denote by $M^{\mathrm{c}}:=\left(\beta_{N}^{\mathrm{c}}\right)^{2} \tau_{v}^{-1}+(1 / N)\left(\beta_{N}^{\mathrm{c}}\right)^{2} \tau_{e}^{-1}+\sigma_{z_{0}}^{2}$ and by $M:=\beta_{N}^{2} \tau_{v}^{-1}+(1 / N) \beta_{N}^{2} \tau_{e}^{-1}+$ $\sigma_{z_{0}}^{2}$. Then as in the proof of Proposition 2 we obtain

$$
\tilde{p}_{N}-\tilde{p}_{N}^{\mathrm{c}}=a \tilde{v}+a \frac{1}{N} \sum_{n=1}^{N} \tilde{e}_{n}+b \tilde{z}_{0}
$$

where

$$
a:=\left(M M^{\mathrm{c}}\right)^{-1}\left(\left(\beta_{N}^{2}-\left(\beta_{N}^{\mathrm{c}}\right)^{2}\right) \tau_{v}^{-1} \sigma_{z_{0}}^{2}\right) \propto\left(\beta_{N}-\beta_{N}^{\mathrm{c}}\right) \sigma_{z_{0}}^{2} \propto \frac{N}{\sigma_{z}^{2}(N)} \frac{\sigma_{z}^{2}(N)}{N^{2}} \propto \frac{1}{N}
$$

and

$$
b:=\left(M M^{\mathrm{c}}\right)^{-1}\left(\beta_{N} \beta_{N}^{\mathrm{c}}\left(\beta_{N}^{\mathrm{c}}-\beta_{N}\right)\left(\tau_{v}^{-1}+\frac{1}{N} \tau_{e}^{-1}\right) \tau_{v}^{-1}+\left(\beta_{N}-\beta_{N}^{\mathrm{c}}\right) \tau_{v}^{-1} \sigma_{z_{0}}^{2}\right) .
$$

So $b \propto\left(\beta_{N}-\beta_{N}^{\mathrm{c}}\right) \propto N / \sigma_{z}^{2}(N)$. Thus,

$$
\operatorname{var}\left[\tilde{p}_{N}-\tilde{p}_{N}^{\mathrm{c}}\right]=a^{2} \tau_{v}^{-1}+a^{2} \frac{1}{N} \tau_{e}^{-1}+b^{2} \sigma_{z_{0}}^{2} \propto\left(\frac{N^{2}}{\sigma_{z}^{4}(N)}\right)\left(\frac{\sigma_{z}^{2}(N)}{N^{2}}\right) \propto\left(\frac{1}{\sigma_{z}^{2}(N)}\right)
$$


Proof of Proposition 8 Let $\tilde{z}_{0}:=\tilde{z}(N) / N$. Then $\sigma_{z_{0}}^{2}:=\operatorname{var}\left[\tilde{z}_{0}\right] \propto 1 / N$. From (9) we obtain

$$
\begin{aligned}
& \tilde{p}_{N}^{\mathrm{c}}=\frac{\tau_{v}^{-1}}{\tau_{v}^{-1}+(1 / N) \tau_{e}^{-1}+\sigma_{z_{0}}^{2} /\left(\beta_{N}^{\mathrm{c}}\right)^{2}}\left(\tilde{v}+\frac{1}{N} \sum_{n=1}^{N} \tilde{e}_{n}+\frac{\tilde{z}_{0}}{\beta_{N}^{\mathrm{c}}}\right) \\
& \tilde{p}_{N}=\frac{\tau_{v}^{-1}}{\tau_{v}^{-1}+(1 / N) \tau_{e}^{-1}+\left(\sigma_{z_{0}}^{2} / \beta_{N}^{2}\right)}\left(\tilde{v}+\frac{1}{N} \sum_{n=1}^{N} \tilde{e}_{n}+\frac{\tilde{z}_{0}}{\beta_{N}}\right) .
\end{aligned}
$$

Then the (equal) left-hand sides of (8) converge to a non-zero constant as $N$ grows. Thus, the right-hand sides also should converge to a non-zero constant. Hence, $\varphi_{\mathrm{I}}^{\mathrm{c}}$ and $\varphi_{\mathrm{I}}$ converge to two different constants, say $\left(\varphi_{\mathrm{I}}^{\mathrm{c}}\right)_{\infty}$ and $\left(\varphi_{\mathrm{I}}\right)_{\infty}$. Then $(8)$ implies that $\left(\varphi_{\mathrm{I}}^{\mathrm{c}}\right)_{\infty}>\left(\varphi_{\mathrm{I}}\right)_{\infty}$. (This is also a conclusion of Theorem 7.1 in Kyle (1989).) Moreover, $\left(1-\varphi_{\mathrm{I}}^{\mathrm{c}}\right),\left(1-\varphi_{\mathrm{I}}\right)$ and $(1-2 \zeta)$ do not converge to zero. Therefore, it follows from (5) that $\beta_{N}^{\mathrm{c}}$ and $\beta_{N}$ also converge to some non-zero constants. Then from (2) we obtain

$$
\varphi_{\mathrm{I}}-\varphi_{\mathrm{I}}^{\mathrm{c}}=\frac{(N-1) \sigma_{z}^{2} \tau_{e}\left(\beta_{N}^{2}-\left(\beta_{N}^{\mathrm{c}}\right)^{2}\right)}{\left((N-1) \beta_{N}^{2}+\sigma_{z}^{2} \tau_{e}\right)\left((N-1)\left(\beta_{N}^{\mathrm{c}}\right)^{2}+\sigma_{z}^{2} \tau_{e}\right)} \propto \frac{N^{2}\left(\beta_{N}-\beta_{N}^{\mathrm{c}}\right)}{N^{2}} \propto\left(\beta_{N}-\beta_{N}^{\mathrm{c}}\right)
$$

Thus, $\left(\beta_{N}-\beta_{N}^{\mathrm{c}}\right)$ also converge to a non-zero constant. Direct computation shows that

$$
\begin{aligned}
\operatorname{var}\left[\tilde{p}_{N}^{\mathrm{c}}-\tilde{v}\right] & =\left(\frac{\tau_{v}^{-1}}{\tau_{v}^{-1}+(1 / N) \tau_{e}^{-1}+\sigma_{z_{0}}^{2} /\left(\beta_{N}^{\mathrm{c}}\right)^{2}}\right)^{2}\left(\tau_{e}^{-1} \frac{1}{N}+\frac{\sigma_{z_{0}}^{2}}{\left(\beta_{N}^{\mathrm{c}}\right)^{2}}\right)+o\left(\frac{1}{N}\right), \\
\operatorname{var}\left[\tilde{p}_{N}-\tilde{v}\right] & =\left(\frac{\tau_{v}^{-1}}{\tau_{v}^{-1}+(1 / N) \tau_{e}^{-1}+\sigma_{z_{0}}^{2} / \beta_{N}^{2}}\right)^{2}\left(\tau_{e}^{-1} \frac{1}{N}+\frac{\sigma_{z_{0}}^{2}}{\left(\beta_{N}\right)^{2}}\right)+o\left(\frac{1}{N}\right), \\
\operatorname{var}\left[\tilde{p}_{N}-\tilde{p}_{N}^{\mathrm{c}}\right] & =\left(\frac{\tau_{v}^{-1} / \beta_{N}}{\tau_{v}^{-1}+(1 / N) \tau_{e}^{-1}+\sigma_{z_{0}}^{2} / \beta_{N}^{2}}-\frac{\tau_{v}^{-1} / \beta_{N}^{\mathrm{c}}}{\tau_{v}^{-1}+(1 / N) \tau_{e}^{-1}+\sigma_{z_{0}}^{2} / \beta_{N}^{2}}\right)^{2} \sigma_{z_{0}}^{2}+o\left(\frac{1}{N}\right) .
\end{aligned}
$$

However,

$$
\left(\frac{\tau_{v}^{-1} / \beta_{N}}{\tau_{v}^{-1}+(1 / N) \tau_{e}^{-1}+\sigma_{z_{0}}^{2} / \beta_{N}^{2}}-\frac{\tau_{v}^{-1} / \beta_{N}^{\mathrm{c}}}{\tau_{v}^{-1}+(1 / N) \tau_{e}^{-1}+\sigma_{z_{0}}^{2} / \beta_{N}^{2}}\right)
$$

converges to $\left(1 / \beta_{N}-1 / \beta_{N}^{\mathrm{c}}\right)$, while

$$
\left(\frac{\tau_{v}^{-1}}{\tau_{v}^{-1}+(1 / N) \tau_{e}^{-1}+\sigma_{z_{0}}^{2} /\left(\beta_{N}^{\mathrm{c}}\right)^{2}}\right) \quad \text { and } \quad\left(\frac{\tau_{v}^{-1}}{\tau_{v}^{-1}+(1 / N) \tau_{e}^{-1}+\sigma_{z_{0}}^{2} / \beta_{N}^{2}}\right)
$$


converge to one. Hence,

$$
\begin{aligned}
\operatorname{var}\left[\tilde{p}_{N}^{\mathrm{c}}-\tilde{v}\right] & =\tau_{e}^{-1} \frac{1}{N}+\frac{\sigma_{z_{0}}^{2}}{\left(\beta_{N}^{\mathrm{c}}\right)^{2}}+o\left(\frac{1}{N}\right), \\
\operatorname{var}\left[\tilde{p}_{N}-\tilde{v}\right] & =\tau_{e}^{-1} \frac{1}{N}+\frac{\sigma_{z_{0}}^{2}}{\left(\beta_{N}\right)^{2}}+o\left(\frac{1}{N}\right), \\
\operatorname{var}\left[\tilde{p}_{N}-\tilde{p}_{N}^{\mathrm{c}}\right] & =\left(\frac{\sigma_{z_{0}}}{\beta_{N}}-\frac{\sigma_{z_{0}}}{\beta_{N}^{\mathrm{c}}}\right)^{2}+o\left(\frac{1}{N}\right) .
\end{aligned}
$$

Expressions (5) and (8) imply that $N\left(\sigma_{z_{0}}^{2} /\left(\beta_{N}^{\mathrm{c}}\right)^{2}\right)$ converges to $\tau_{e}^{-1}\left(\left(1-\left(\varphi_{\mathrm{I}}^{\mathrm{c}}\right)_{\infty}\right) /\left(\varphi_{\mathrm{I}}^{\mathrm{c}}\right)_{\infty}\right)$, while $N\left(\sigma_{z_{0}}^{2} /\left(\beta_{N}\right)^{2}\right)$ converges to $\tau_{e}^{-1}\left(\left(1-\left(\varphi_{\mathrm{I}}\right)_{\infty}\right) /\left(\varphi_{\mathrm{I}}\right)_{\infty}\right)$. Let us define

$$
x:=\sqrt{\frac{1-\left(\varphi_{\mathrm{I}}\right)_{\infty}}{\left(\varphi_{\mathrm{I}}\right)_{\infty}}} \text { and } \quad x_{\mathrm{c}}:=\sqrt{\frac{1-\left(\varphi_{\mathrm{I}}^{\mathrm{c}}\right)_{\infty}}{\left(\varphi_{\mathrm{I}}^{\mathrm{c}}\right)_{\infty}}} .
$$

Recall that $\left(\varphi_{\mathrm{I}}^{\mathrm{c}}\right)_{\infty}>\left(\varphi_{\mathrm{I}}\right)_{\infty}$. Hence, $x>x_{\mathrm{c}}$. Then

$$
\begin{aligned}
\operatorname{var}\left[\tilde{p}_{N}^{\mathrm{c}}-\tilde{v}\right] & =\tau_{e}^{-1}\left(1+x_{\mathrm{c}}^{2}\right) \frac{1}{N}+o\left(\frac{1}{N}\right), \\
\operatorname{var}\left[\tilde{p}_{N}-\tilde{v}\right] & =\tau_{e}^{-1}\left(1+x^{2}\right) \frac{1}{N}+o\left(\frac{1}{N}\right), \\
\operatorname{var}\left[\tilde{p}_{N}-\tilde{p}_{N}^{\mathrm{c}}\right] & =\tau_{e}^{-1}\left(x-x_{\mathrm{c}}\right)^{2} \frac{1}{N}+o\left(\frac{1}{N}\right) .
\end{aligned}
$$

Since $1+x^{2}>1+x_{\mathrm{c}}^{2}$ and $1+x^{2}>x^{2}>\left(x-x_{\mathrm{c}}\right)^{2}$, the proposition is proved.

Proof of Proposition 9 Let $\tilde{z}_{0}:=\tilde{z}(N) / N$. Then $\sigma_{z_{0}}^{2}:=\operatorname{var}\left[\tilde{z}_{0}\right]=\sigma_{z}^{2}(N) / N^{2}$. From $(9)$ we obtain

$$
\begin{aligned}
& \tilde{p}_{N}^{\mathrm{c}}=\frac{\tau_{v}^{-1}}{\tau_{v}^{-1}+(1 / N) \tau_{e}^{-1}+\sigma_{z_{0}}^{2} /\left(\beta_{N}^{\mathrm{c}}\right)^{2}}\left(\tilde{v}+\frac{1}{N} \sum_{n=1}^{N} \tilde{e}_{n}+\frac{\tilde{z}_{0}}{\beta_{N}^{\mathrm{c}}}\right) \\
& \tilde{p}_{N}=\frac{\tau_{v}^{-1}}{\tau_{v}^{-1}+(1 / N) \tau_{e}^{-1}+\sigma_{z_{0}}^{2} / \beta_{N}^{2}}\left(\tilde{v}+\frac{1}{N} \sum_{n=1}^{N} \tilde{e}_{n}+\frac{\tilde{z}_{0}}{\beta_{N}}\right)
\end{aligned}
$$

Then an analysis of (8) very similar to that in the proof of Proposition 8 shows that $\varphi_{\mathrm{I}}^{\mathrm{c}}$ converge to one at a rate of $\left(\sigma_{z}^{2}(N) / N\right)^{1 / 3}$, and that $\varphi_{\mathrm{I}}$ and $\zeta$ converge to $1 / 2$ at a rate of $\left(\sigma_{z}^{2}(N) / N\right)^{1 / 2}$. (Since $\zeta \leq 1 / 2$, by $(6)$ we obtain that $\varphi_{\mathrm{I}}$ and $\zeta$ converge to $1 / 2$.) Then from (5) we obtain that $\beta_{N}^{\mathrm{c}} \propto\left(\sigma_{z}^{2}(N) / N\right)^{1 / 3}$ and $\beta_{N} \propto\left(\sigma_{z}^{2}(N) / N\right)^{1 / 2}$. Thus $\left(\beta-\beta^{\mathrm{c}}\right) \propto$ $\left(\sigma_{z}^{2}(N) / N\right)^{1 / 3}$. Moreover, $\sigma_{z_{0}}^{2} /\left(\beta_{N}^{\mathrm{c}}\right)^{2} \propto\left(\sigma_{z}^{2}(N) / N\right)^{1 / 3}(1 / N)=o(1 / N)$ and $\sigma_{z_{0}}^{2} / \beta_{N}^{2} \propto 1 / N$. 
Direct computation shows that

$$
\begin{aligned}
\operatorname{var}\left[\tilde{p}_{N}^{\mathrm{c}}-\tilde{v}\right] & =\left(\frac{\tau_{v}^{-1}}{\tau_{v}^{-1}+(1 / N) \tau_{e}^{-1}+\sigma_{z_{0}}^{2} /\left(\beta_{N}^{\mathrm{c}}\right)^{2}}\right)^{2} \tau_{e}^{-1} \frac{1}{N}+o\left(\frac{1}{N}\right), \\
\operatorname{var}\left[\tilde{p}_{N}-\tilde{v}\right] & =\left(\frac{\tau_{v}^{-1}}{\tau_{v}^{-1}+(1 / N) \tau_{e}^{-1}+\sigma_{z_{0}}^{2} / \beta_{N}^{2}}\right)^{2}\left(\tau_{e}^{-1} \frac{1}{N}+\frac{\sigma_{z_{0}}^{2}}{\left(\beta_{N}\right)^{2}}\right)+o\left(\frac{1}{N}\right), \\
\operatorname{var}\left[\tilde{p}_{N}-\tilde{p}_{N}^{\mathrm{c}}\right] & =\left(\frac{\tau_{v}^{-1}}{\tau_{v}^{-1}+(1 / N) \tau_{e}^{-1}+\sigma_{z_{0}}^{2} / \beta_{N}^{2}}\right)^{2} \frac{\sigma_{z_{0}}^{2}}{\left(\beta_{N}\right)^{2}}+o\left(\frac{1}{N}\right) .
\end{aligned}
$$

However, $\left.\left(\tau_{v}^{-1} /\left(\tau_{v}^{-1}+(1 / N) \tau_{e}^{-1}+\sigma_{z_{0}}^{2} /\left(\beta_{N}^{\mathrm{c}}\right)^{2}\right)\right)\right)$ converges to one, while (5) and (8) imply that $N\left(\sigma_{z_{0}}^{2} /\left(\beta_{N}\right)^{2}\right)$ converges to $\tau_{e}^{-1}$. This proves the conclusions of the proposition.

Proof of Proposition 10 If $N$ grows at a slower rate than $\sigma_{z}$, then from the characterization (8) we obtain that $\varphi_{\mathrm{I}}^{\mathrm{c}}$ and $\varphi_{\mathrm{I}}$ converge to zero at a rate of $N / \sigma_{z}^{2}$ Then it follows from (3) that $\varphi_{\mathrm{U}}^{\mathrm{c}}$ and $\varphi_{\mathrm{U}}$ also converge to zero at a rate of $N / \sigma_{z}^{2}$. Hence, $\tau_{\mathrm{U}}^{\mathrm{c}}=\tau_{v}+\varphi_{\mathrm{U}}^{\mathrm{c}} N \tau_{e}$ and $\tau_{\mathrm{U}}=\tau_{v}+\varphi_{\mathrm{U}} N \tau_{e}$ converge to $\tau_{v}$, while $\tau_{\mathrm{I}}^{\mathrm{c}}$ and $\tau_{\mathrm{I}}$ converge to $\tau_{v}+\tau_{e}$. Then from (7) we obtain that $\zeta$ converges to zero at a rate of $N / \sigma_{z}^{2}$. Then, as in the proof of Lemma 1 ,

$$
\frac{\left(1-\varphi_{\mathrm{I}}\right)^{3}}{\left(1-\varphi_{\mathrm{I}}^{\mathrm{c}}\right)^{3}} \frac{\varphi_{\mathrm{I}}^{\mathrm{c}}}{\varphi_{\mathrm{I}}}-1=\frac{\zeta(2-3 \zeta)}{(1-\zeta)^{2}} \propto \frac{N}{\sigma_{z}^{2}(N)}
$$

and

$$
\begin{aligned}
\frac{\left(1-\varphi_{\mathrm{I}}\right)^{3}}{\left(1-\varphi_{\mathrm{I}}^{\mathrm{c}}\right)^{3}} \frac{\varphi_{\mathrm{I}}^{\mathrm{c}}}{\varphi_{\mathrm{I}}}-1 & =\frac{\left(1-\varphi_{\mathrm{I}}\right)^{3}}{\left(1-\varphi_{\mathrm{I}}^{\mathrm{c}}\right)^{3}}\left(\frac{\varphi_{\mathrm{I}}^{\mathrm{c}}}{\varphi_{\mathrm{I}}}-1\right)+\left(\frac{\left(1-\varphi_{\mathrm{I}}\right)^{3}}{\left(1-\varphi_{\mathrm{I}}^{\mathrm{c}}\right)^{3}}-1\right) \\
& \propto \varphi_{\mathrm{I}}^{-1}\left(\varphi_{\mathrm{I}}-\varphi_{\mathrm{I}}^{\mathrm{c}}\right)+O\left(\varphi_{\mathrm{I}}-\varphi_{\mathrm{I}}^{\mathrm{c}}\right) \propto \frac{\sigma_{z}^{2}(N)}{N}\left(\varphi_{\mathrm{I}}-\varphi_{\mathrm{I}}^{\mathrm{c}}\right) .
\end{aligned}
$$

Hence, $\left(\varphi_{\mathrm{I}}\right)_{N}-\left(\varphi_{\mathrm{I}}^{\mathrm{c}}\right)_{N} \propto N^{2} / \sigma_{z}^{4}(N)$. Then $\beta_{N}$ and $\beta_{N}^{\mathrm{c}}$ converge to $\beta_{\infty}=\tau_{e} / \rho$ and, from (2), we obtain

$$
\varphi_{\mathrm{I}}-\varphi_{\mathrm{I}}^{\mathrm{c}}=\frac{(N-1) \sigma_{z}^{2} \tau_{e}\left(\beta_{N}^{2}-\left(\beta_{N}^{\mathrm{c}}\right)^{2}\right)}{\left((N-1) \beta_{N}^{2}+\sigma_{z}^{2} \tau_{e}\right)\left((N-1)\left(\beta_{N}^{\mathrm{c}}\right)^{2}+\sigma_{z}^{2} \tau_{e}\right)} \propto \frac{N \sigma_{z}^{2}(N)\left(\beta_{N}-\beta_{N}^{\mathrm{c}}\right)}{\sigma_{z}^{4}(N)} .
$$

Thus, $\beta_{N}-\beta_{N}^{\mathrm{c}} \propto N / \sigma_{z}^{2}(N)$. Let us define $\tilde{z}_{0}:=\tilde{z}(N) / N$. Then $\sigma_{z_{0}}^{2}:=\operatorname{var}\left[\tilde{z}_{0}\right]=\sigma_{z}^{2}(N) / N^{2}$ is growing with $N$. Now let us denote by $M^{\mathrm{c}}:=\left(\beta_{N}^{\mathrm{c}}\right)^{2} \tau_{v}^{-1}+(1 / N)\left(\beta_{N}^{\mathrm{c}}\right)^{2} \tau_{e}^{-1}+\sigma_{z_{0}}^{2}$ and by $M:=\beta_{N}^{2} \tau_{v}^{-1}+(1 / N) \beta_{N}^{2} \tau_{e}^{-1}+\sigma_{z_{0}}^{2}$. Note that $M^{\mathrm{c}} \propto M \propto \sigma_{z_{0}}^{2}$. Then, as in the proof of Proposition 2, we obtain

$$
\tilde{p}_{N}-\tilde{p}_{N}^{\mathrm{c}}=a \tilde{v}+a \frac{1}{N} \sum_{n=1}^{N} \tilde{e}_{n}+b \tilde{z}_{0}
$$


where

$$
a:=\left(M M^{\mathrm{c}}\right)^{-1}\left(\left(\beta_{N}^{2}-\left(\beta_{N}^{\mathrm{c}}\right)^{2}\right) \tau_{v}^{-1} \sigma_{z_{0}}^{2}\right) \propto\left(\beta_{N}-\beta_{N}^{\mathrm{c}}\right) / \sigma_{z_{0}}^{2} \propto \frac{N}{\sigma_{z}^{2}(N)} \frac{N^{2}}{\sigma_{z}^{2}(N)} \propto \frac{N^{3}}{\sigma_{z}^{4}(N)}
$$

and

$$
b:=\left(M M^{\mathrm{c}}\right)^{-1}\left(\beta_{N} \beta_{N}^{\mathrm{c}}\left(\beta_{N}^{\mathrm{c}}-\beta_{N}\right)\left(\tau_{v}^{-1}+\frac{1}{N} \tau_{e}^{-1}\right) \tau_{v}^{-1}+\left(\beta_{N}-\beta_{N}^{\mathrm{c}}\right) \tau_{v}^{-1} \sigma_{z_{0}}^{2}\right) .
$$

So $b \propto\left(\beta_{N}-\beta_{N}^{\text {c }}\right) / \sigma_{z_{0}}^{2} \propto N^{3} / \sigma_{z}^{4}(N)$. Thus,

$$
\operatorname{var}\left[\tilde{p}_{N}-\tilde{p}_{N}^{\mathrm{c}}\right]=a^{2} \tau_{v}^{-1}+a^{2} \frac{1}{N} \tau_{e}^{-1}+b^{2} \sigma_{z_{0}}^{2} \propto\left(\frac{N^{3}}{\sigma_{z}^{4}(N)}\right)^{2}\left(\frac{\sigma_{z}^{2}(N)}{N^{2}}\right) \propto \frac{N^{4}}{\sigma_{z}^{6}(N)}
$$

This proves part 1 of the proposition. Inspection of expressions (9) shows that in the case described in Proposition 10

$$
\operatorname{var}\left[\tilde{p}_{N}^{\mathrm{c}}\right] \propto \operatorname{var}\left[\tilde{p}_{N}\right] \propto \operatorname{var}\left[\frac{\tilde{z}_{0}}{\sigma_{z_{0}}^{2}}\right] \propto \frac{1}{\sigma_{z}^{2}(N)} \propto \frac{N^{2}}{\sigma_{z}^{2}(N)}
$$

This proves parts 2 and 3 of the proposition.

Proof of Proposition 11 Let us start with an exogenously given number of informed speculators $N$. Expressions (8) and (6) imply, for $\rho$ converging zero, that $\varphi_{\mathrm{I}}^{\mathrm{c}} \rightarrow 1, \varphi_{\mathrm{I}} \rightarrow 1 / 2$ and $\zeta \rightarrow 1 / 2$. Then, by (3), $\varphi_{\mathrm{U}}^{\mathrm{c}} \rightarrow 1$ and $\varphi_{\mathrm{U}} \rightarrow 1 / 2$. Moreover, both $\varphi_{\mathrm{I}}^{\mathrm{c}} \rightarrow 1$ and $\varphi_{\mathrm{U}}^{\mathrm{c}} \rightarrow 1$ at a rate of $\rho^{2 / 3}$. Applying L'Hôpital's rule to the expression for the certainty equivalents of profits given by Kyle (1989, Theorem 10.1), we obtain

$$
\Pi^{\mathrm{c}}(N)=\frac{1}{2 \rho} \log \left(1+\frac{\left(1-\varphi_{\mathrm{I}}^{\mathrm{c}}\right)\left(1-\varphi_{\mathrm{U}}^{\mathrm{c}}\right) \tau_{e}}{\tau_{\mathrm{U}}^{\mathrm{c}}}\right) \propto \rho^{1 / 3} \rightarrow 0 \quad \text { as } \rho \rightarrow 0 .
$$

Therefore, $N_{\mathrm{c}}^{*}\left(\sigma_{z}\right)=0$ for $\rho \rightarrow 0$. Expression (2) implies that $N\left(\beta_{N}\right)^{2} /\left(N\left(\beta_{N}\right)^{2}+\sigma_{z}^{2} \tau_{e}\right)=$ $\varphi_{\mathrm{U}} \rightarrow 1 / 2$ for $\rho \rightarrow 0$. Since $\sigma_{z}$ is fixed for a given $N$, it follows that $N\left(\beta_{N}\right)^{2} \rightarrow \sigma_{z}^{2} \tau_{e}$ for $\rho \rightarrow 0$. Therefore,

$$
\beta_{N} \rightarrow \sigma_{z} \sqrt{\frac{\tau_{e}}{N}} \text { for } \rho \rightarrow 0 .
$$

Applying L'Hôpital's rule to the expression for the certainty equivalents of profits given by Kyle (1989, Theorem 10.1), we obtain

$$
\Pi(N)=\frac{1}{2 \rho} \log \left(1+\frac{\rho \beta_{N}\left(1-\varphi_{\mathrm{U}}\right)}{\tau_{\mathrm{U}}}\right) \rightarrow \frac{\sigma_{z} \sqrt{\tau_{e}}}{\left(4 \tau_{v}+2 N \tau_{v}\right) \sqrt{N}} \equiv \Pi_{0}(N) \quad \text { as } \rho \rightarrow 0 .
$$

We have that $\Pi_{0}(N) \rightarrow 0$ as $N \rightarrow \infty$. We know that $\Pi_{0}\left(N^{*}\left(\sigma_{z}\right)\right)$ will be approximately equal to $F\left(\sigma_{z}\right)$ (and the approximation will be good for $\sigma_{z}$ large). Therefore, $\lim _{\rho \rightarrow 0} N^{*}\left(\sigma_{z}\right) \propto$ $\left(\sigma_{z} / F\left(\sigma_{z}\right)\right)^{2 / 3}$. The strategic equilibrium allows a direct analysis of the risk-neutral case. 
Moreover, by continuity the same results have to be obtained for $\rho \rightarrow 0$ and $\rho=0$.

Proof of Proposition 12 As in the proof of Proposition 1, let us start with the exogenously given number of informed speculators $N$. Kyle (1989, Theorem 10.1) provides the following expressions for the certainty equivalents of profits:

$$
\begin{aligned}
\Pi^{\mathrm{c}}(N) & =\frac{1}{2 \rho} \log \left(1+\frac{\left(1-\varphi_{\mathrm{I}}^{\mathrm{c}}\right)\left(1-\varphi_{\mathrm{U}}^{\mathrm{c}}\right) \tau_{e}}{\tau_{\mathrm{U}}^{\mathrm{c}}}\right), \\
\Pi(N) & =\frac{1}{2 \rho} \log \left(1+\frac{\left(1-\varphi_{\mathrm{I}}\right)\left(1-\varphi_{\mathrm{U}}\right) \tau_{e}}{\tau_{\mathrm{U}}} \frac{(1-2 \zeta)}{(1-\zeta)^{2}}\right) .
\end{aligned}
$$

Since $N \tau_{e}=\tau_{E}$, the (equal) left-hand sides of (8) converge to a non-zero constant as $N$ grows. Thus, the right-hand sides also should converge to a non-zero constant. Thus, there exist two different non-zero constants $\left(\varphi_{\mathrm{I}}^{\mathrm{c}}\right)_{\infty}$ and $\left(\varphi_{\mathrm{I}}\right)_{\infty}$, such that $\left(\varphi_{\mathrm{I}}^{\mathrm{c}}\right)_{N} \rightarrow\left(\varphi_{\mathrm{I}}^{\mathrm{c}}\right)_{\infty}$ and $\left(\varphi_{\mathrm{I}}\right)_{N} \rightarrow\left(\varphi_{\mathrm{I}}\right)_{\infty}$ as $N \rightarrow \infty$. Similarly, there exist $\left(\varphi_{\mathrm{U}}^{\mathrm{c}}\right)_{\infty},\left(\varphi_{\mathrm{U}}\right)_{\infty}$ and $\zeta_{\infty}$. Moreover, $\left(1-\left(\varphi_{\mathrm{I}}^{\mathrm{c}}\right)_{\infty}\right),\left(1-\left(\varphi_{\mathrm{I}}\right)_{\infty}\right)$, $\left(1-\left(\varphi_{\mathrm{U}}^{\mathrm{c}}\right)_{\infty}\right),\left(1-\left(\varphi_{\mathrm{U}}\right)_{\infty}\right)$ and $\left(1-2 \zeta_{\infty}\right)$ are all non-zero constants. Therefore, since $\tau_{\mathrm{U}}^{\mathrm{c}}$ and $\tau_{\mathrm{U}}$ are bounded, both $\Pi^{\mathrm{c}}(N)$ and $\Pi(N)$ converge to zero. This implies the conclusions of the proposition.

Let us state a lemma before proving Proposition 13.

Lemma 5. For the monopolistic competition case

$$
\xi_{\mathrm{I}} N=\frac{\tau_{v}+\tau_{E}}{\left(\tau_{v} / \varphi_{\mathrm{U}}\right)+\tau_{E}} .
$$

Proof. Condition (B.22) in Kyle (1989) states that

$$
\zeta \tau_{\mathrm{U}}-\varphi_{\mathrm{U}} \tau_{\mathrm{I}}=\left(1-\varphi_{\mathrm{I}}\right)\left(\left(\xi_{\mathrm{I}}-\frac{\varphi_{\mathrm{U}}}{N}\right) \tau_{v}-\varphi_{\mathrm{U}}\left(1-N \xi_{\mathrm{I}}\right) \tau_{e}\right)
$$

Since the left-hand side of this expression is zero by (7) and $\varphi_{\mathrm{I}}<1$, we should obtain $\left(\xi_{\mathrm{I}}-\varphi_{\mathrm{U}} / N\right) \tau_{v}-\varphi_{\mathrm{U}}\left(1-N \xi_{\mathrm{I}}\right) \tau_{e}=0$. Therefore, $\left(N \xi_{\mathrm{I}}-\varphi_{\mathrm{U}}\right) \tau_{v}-\varphi_{\mathrm{U}}\left(1-N \xi_{\mathrm{I}}\right) \tau_{E}=0$. However, this is equivalent to $N \xi_{\mathrm{I}}\left(\tau_{v}+\varphi_{\mathrm{U}} \tau_{E}\right)=\varphi_{\mathrm{U}}\left(\tau_{v}+\tau_{E}\right)$. Thus $N \xi_{\mathrm{I}}=\left(\varphi_{\mathrm{U}}\left(\tau_{v}+\tau_{E}\right)\right) /\left(\tau_{v}+\varphi_{\mathrm{U}} \tau_{E}\right)=$ $\left(\tau_{v}+\tau_{E}\right) /\left(\left(\tau_{v} / \varphi_{\mathrm{U}}\right)+\tau_{E}\right)$.

Proof of Proposition 13 From formulas (4) we obtain the following expressions for prices in two equilibria in the $N$ th market:

$$
\begin{aligned}
& \tilde{p}_{N}^{\mathrm{c}}=\frac{\tau_{v}^{-1}}{\tau_{v}^{-1}+\tau_{E}^{-1}+\left(1 / \beta_{N}^{\mathrm{c}} N\right)^{2} \sigma_{z}^{2}}\left(\tilde{v}+\tilde{e}+\frac{1}{\beta_{N}^{\mathrm{c}} N} \tilde{z}\right), \\
& \tilde{p}_{N}=\frac{\tau_{v}^{-1}}{\tau_{v}^{-1}+\tau_{E}^{-1}+\left(1 / \beta_{N} N\right)^{2} \sigma_{z}^{2}}\left(\tilde{v}+\tilde{e}+\frac{1}{\beta_{N} N} \tilde{z}\right) .
\end{aligned}
$$

The proof proceeds in four steps. (1) Let us prove that there exist different non-zero constants 
$d$ and $d^{\mathrm{c}}$ such that

$$
\begin{aligned}
& \tilde{p}_{\infty}^{\mathrm{c}}=\frac{\tau_{v}^{-1}}{\tau_{v}^{-1}+\tau_{E}^{-1}+\left(d^{\mathrm{c}}\right)^{2} \sigma_{z}^{2}}\left(\tilde{v}+\tilde{e}+d^{\mathrm{c}} \tilde{z}\right) \quad \text { and } \\
& \tilde{p}_{\infty}=\frac{\tau_{v}^{-1}}{\tau_{v}^{-1}+\tau_{E}^{-1}+d^{2} \sigma_{z}^{2}}(\tilde{v}+\tilde{e}+d \tilde{z})
\end{aligned}
$$

This will imply that $\tilde{p}_{\infty}^{\mathrm{c}} \neq \tilde{p}_{\infty}$. As in the proof of Proposition 12 , there exist two different non-zero constants $\left(\varphi_{\mathrm{I}}^{\mathrm{c}}\right)_{\infty}$ and $\left(\varphi_{\mathrm{I}}\right)_{\infty}$, such that $\left(\varphi_{\mathrm{I}}^{\mathrm{c}}\right)_{N} \rightarrow\left(\varphi_{\mathrm{I}}^{\mathrm{c}}\right)_{\infty}$ and $\left(\varphi_{\mathrm{I}}\right)_{N} \rightarrow\left(\varphi_{\mathrm{I}}\right)_{\infty}$ as $N \rightarrow \infty$. Moreover $\left(1-\left(\varphi_{\mathrm{I}}^{\mathrm{c}}\right)_{\infty}\right),\left(1-\left(\varphi_{\mathrm{I}}\right)_{\infty}\right)$ and $\left(1-2 \zeta_{\infty}\right)$ are all non-zero constants. Thus, it follows from (5) that there exist non-zero constants $d$ and $d^{\mathrm{c}}$ such that $1 / \beta_{N}^{\mathrm{c}} N \rightarrow d^{\mathrm{c}}$ and $1 / \beta_{N} N \rightarrow d$. So we proved formulas for the limit prices. Now from (2) we obtain

$$
\begin{aligned}
\varphi_{\mathrm{I}}-\varphi_{\mathrm{I}}^{\mathrm{c}} & =\frac{(N-1) \sigma_{z}^{2} \tau_{e}\left(\beta_{N}^{2}-\left(\beta_{N}^{\mathrm{c}}\right)^{2}\right)}{\left((N-1) \beta_{N}^{2}+\sigma_{z}^{2} \tau_{e}\right)\left((N-1)\left(\beta_{N}^{\mathrm{c}}\right)^{2}+\sigma_{z}^{2} \tau_{e}\right)} \\
& \propto \frac{\left(\beta_{N}-\beta_{N}^{\mathrm{c}}\right)(1 / N)}{(1 / N)(1 / N)} \propto\left(\beta_{N} N-\beta_{N}^{\mathrm{c}} N\right) .
\end{aligned}
$$

Since $\left(\varphi_{\mathrm{I}}^{\mathrm{c}}\right)_{\infty} \neq\left(\varphi_{\mathrm{I}}\right)_{\infty}$, we have that $d \neq d^{\mathrm{c}}$. (2) Now let us prove the statement of the proposition for competitive equilibrium. Let us first introduce some notation. Let

$$
k_{N}^{\mathrm{c}}:=\frac{\tau_{v}^{-1}}{\tau_{v}^{-1}+\tau_{E}^{-1}+\left(1 / \beta_{N}^{\mathrm{c}} N\right)^{2} \sigma_{z}^{2}} \quad \text { and } \quad k_{\infty}^{\mathrm{c}}:=\frac{\tau_{v}^{-1}}{\tau_{v}^{-1}+\tau_{E}^{-1}+\left(d^{\mathrm{c}}\right)^{2} \sigma_{z}^{2}} .
$$

Then $k_{N}^{\mathrm{c}}-k_{\infty}^{\mathrm{c}} \propto\left(1 / \beta_{N}^{\mathrm{c}} N\right)^{2}-\left(d^{\mathrm{c}}\right)^{2} \propto 1 / \beta_{N}^{\mathrm{c}} N-d^{\mathrm{c}}$. Since $\tilde{p}_{N}^{\mathrm{c}}=k_{N}^{\mathrm{c}}\left(\tilde{v}+\tilde{e}+\left(1 / \beta_{N}^{\mathrm{c}} N\right) \tilde{z}\right)$ and $\tilde{p}_{\infty}^{\mathrm{c}}=k_{\infty}^{\mathrm{c}}\left(\tilde{v}+\tilde{e}+d^{\mathrm{c}} \tilde{z}\right)$, we obtain $\tilde{p}_{N}^{\mathrm{c}}-\tilde{p}_{\infty}^{\mathrm{c}}=\left(k_{N}^{\mathrm{c}}-k_{\infty}^{\mathrm{c}}\right)\left(\tilde{v}+\tilde{e}+\left(1 / \beta_{N}^{\mathrm{c}} N\right) \tilde{z}\right)+k_{\infty}^{\mathrm{c}}\left(1 / \beta_{N}^{\mathrm{c}} N-d^{\mathrm{c}}\right) \tilde{z}$ and, thus,

$$
\operatorname{var}\left[\tilde{p}_{N}^{\mathrm{c}}-\tilde{p}_{\infty}^{\mathrm{c}}\right] \propto\left(\frac{1}{\beta_{N}^{\mathrm{c}} N}-d^{\mathrm{c}}\right)^{2} \propto\left(\beta_{N}^{\mathrm{c}} N-\frac{1}{d^{\mathrm{c}}}\right)^{2} .
$$

Then by (5) we have

$$
\beta_{N}^{\mathrm{c}} N-\frac{1}{d^{\mathrm{c}}} \propto\left(\frac{\tau_{E}}{\rho}\left(1-\left(\varphi_{\mathrm{I}}^{\mathrm{c}}\right)_{N}\right)-\frac{\tau_{E}}{\rho}\left(1-\left(\varphi_{\mathrm{I}}^{\mathrm{c}}\right)_{\infty}\right)\right) \propto\left(\varphi_{\mathrm{I}}^{\mathrm{c}}\right)_{N}-\left(\varphi_{\mathrm{I}}^{\mathrm{c}}\right)_{\infty}
$$

And now we have from (8) that

$$
\frac{\sigma_{z}^{2} \rho^{2}}{\tau_{E}} \frac{N}{N-1}=\frac{\left(1-\left(\varphi_{\mathrm{I}}^{\mathrm{c}}\right)_{N}\right)^{3}}{\left(\varphi_{\mathrm{I}}^{\mathrm{c}}\right)_{N}} \quad \text { and } \quad \frac{\sigma_{z}^{2} \rho^{2}}{\tau_{E}}=\frac{\left(1-\left(\varphi_{\mathrm{I}}^{\mathrm{c}}\right)_{\infty}\right)^{3}}{\left(\varphi_{\mathrm{I}}^{\mathrm{c}}\right)_{\infty}}
$$

Therefore,

$$
\frac{\left(1-\left(\varphi_{\mathrm{I}}^{\mathrm{c}}\right)_{\infty}\right)^{3}}{\left(1-\left(\varphi_{\mathrm{I}}^{\mathrm{c}}\right)_{N}\right)^{3}} \frac{\left(\varphi_{\mathrm{I}}^{\mathrm{c}}\right)_{N}}{\left(\varphi_{\mathrm{I}}^{\mathrm{c}}\right)_{\infty}}-1 \propto 1 / N
$$


However, on the other side,

$$
\begin{aligned}
\frac{\left(1-\left(\varphi_{\mathrm{I}}^{\mathrm{c}}\right)_{\infty}\right)^{3}}{\left(1-\left(\varphi_{\mathrm{I}}^{\mathrm{c}}\right)_{N}\right)^{3}} \frac{\left(\varphi_{\mathrm{I}}^{\mathrm{c}}\right)_{N}}{\left(\varphi_{\mathrm{I}}^{\mathrm{c}}\right)_{\infty}}-1=\frac{\left(1-\left(\varphi_{\mathrm{I}}^{\mathrm{c}}\right)_{\infty}\right)^{3}}{\left(1-\left(\varphi_{\mathrm{I}}^{\mathrm{c}}\right)_{N}\right)^{3}} & \left(\frac{\left(\varphi_{\mathrm{I}}^{\mathrm{c}}\right)_{N}}{\left(\varphi_{\mathrm{I}}^{\mathrm{c}}\right)_{\infty}}-1\right)+\left(\frac{\left(1-\left(\varphi_{\mathrm{I}}^{\mathrm{c}}\right)_{\infty}\right)^{3}}{\left(1-\left(\varphi_{\mathrm{I}}^{\mathrm{c}}\right)_{N}\right)^{3}}-1\right) \\
& \propto\left(\varphi_{\mathrm{I}}^{\mathrm{c}}\right)_{N}-\left(\varphi_{\mathrm{I}}^{\mathrm{c}}\right)_{\infty} .
\end{aligned}
$$

Thus, $\left(\varphi_{\mathrm{I}}^{\mathrm{c}}\right)_{N}-\left(\varphi_{\mathrm{I}}^{\mathrm{c}}\right)_{\infty} \propto 1 / N$ and therefore $\operatorname{var}\left(\tilde{p}_{N}^{\mathrm{c}}-\tilde{p}_{\infty}^{\mathrm{c}}\right) \propto 1 / N^{2}$. (3) Now let us look at the equilibrium with imperfect competition. In the same way as above

$$
\operatorname{var}\left[\tilde{p}_{N}-\tilde{p}_{\infty}\right] \propto\left(\frac{1}{\beta_{N} N}-d\right)^{2} \propto\left(\beta_{N} N-\frac{1}{d}\right)^{2}
$$

Then, by (5),

$$
\beta_{N} N=\frac{\tau_{E}}{\rho}\left(1-\left(\varphi_{\mathrm{I}}\right)_{N}\right) \frac{\left(1-2 \zeta_{N}\right)}{\left(1-\zeta_{N}\right)} \quad \text { and } \quad \frac{1}{d}=\frac{\tau_{E}}{\rho}\left(1-\left(\varphi_{\mathrm{I}}\right)_{\infty}\right) \frac{\left(1-2 \zeta_{\infty}\right)}{\left(1-\zeta_{\infty}\right)}
$$

Then by $(6)\left(1-\zeta_{N}\right)=\left(1-\left(\varphi_{\mathrm{I}}\right)_{N}\right)\left(1-\xi_{\mathrm{I}}\right)$ and $\zeta_{\infty}=\left(\varphi_{\mathrm{I}}\right)_{\infty}$. Thus,

$$
\begin{aligned}
\beta_{N} N-\frac{1}{d} & =\frac{\tau_{E}}{\rho}\left(\frac{\left(1-2 \zeta_{N}\right)}{\left(1-\xi_{\mathrm{I}}\right)}-\left(1-2 \zeta_{\infty}\right)\right) \\
& \propto \frac{\xi_{\mathrm{I}}}{\left(1-\xi_{\mathrm{I}}\right)}\left(1-2 \zeta_{N}\right)+2\left(\zeta_{\infty}-\zeta_{N}\right) .
\end{aligned}
$$

Then $2\left(\zeta_{\infty}-\zeta_{N}\right)=2\left(\left(\varphi_{\mathrm{I}}\right)_{\infty}-\left(\varphi_{\mathrm{I}}\right)_{N}\right)-2 \xi_{\mathrm{I}}\left(1-\left(\varphi_{\mathrm{I}}\right)_{N}\right)$. By Lemma 5 , since $0<\lim _{N \rightarrow \infty} \varphi_{\mathrm{U}}=$ $\left(\varphi_{\mathrm{I}}\right)_{\infty}<1$, we can conclude $\xi_{\mathrm{I}} \propto 1 / N$, and $\left(\xi_{\mathrm{I}} /\left(1-\xi_{\mathrm{I}}\right)\right)\left(1-2 \zeta_{N}\right)=\xi_{\mathrm{I}}\left(1-2\left(\varphi_{\mathrm{I}}\right)_{N}\right)+o(1 / N)$. Therefore, we obtain

$$
\beta_{N} N-\frac{1}{d} \propto 2\left(\left(\varphi_{\mathrm{I}}\right)_{\infty}-\left(\varphi_{\mathrm{I}}\right)_{N}\right)-\xi_{\mathrm{I}}+o\left(\frac{1}{N}\right)
$$

Now let us show that it is impossible to have the case where $1 / N=o\left(\left(\varphi_{\mathrm{I}}\right)_{\infty}-\left(\varphi_{\mathrm{I}}\right)_{N}\right)$. Let us first prove that if $1 / N=o\left(\left(\varphi_{\mathrm{I}}\right)_{\infty}-\left(\varphi_{\mathrm{I}}\right)_{N}\right)$, then

$$
\frac{\left(1-\left(\varphi_{\mathrm{I}}\right)_{N}\right)^{3}}{\left(\varphi_{\mathrm{I}}\right)_{N}} \frac{\left(1-2 \zeta_{N}\right)^{2}}{\left(1-\zeta_{N}\right)^{2}}-\frac{\left(1-\left(\varphi_{\mathrm{I}}\right)_{\infty}\right)^{3}}{\left(\varphi_{\mathrm{I}}\right)_{\infty}} \frac{\left(1-2 \zeta_{\infty}\right)^{2}}{\left(1-\zeta_{\infty}\right)^{2}} \propto\left(\varphi_{\mathrm{I}}\right)_{\infty}-\left(\varphi_{\mathrm{I}}\right)_{N}
$$

Since $\zeta_{N}=\left(\varphi_{\mathrm{I}}\right)_{N}+O(1 / N)$, we obtain

$$
\frac{\left(1-\left(\varphi_{\mathrm{I}}\right)_{N}\right)^{3}}{\left(\varphi_{\mathrm{I}}\right)_{N}} \frac{\left(1-2 \zeta_{N}\right)^{2}}{\left(1-\zeta_{N}\right)^{2}}=\frac{\left(1-\left(\varphi_{\mathrm{I}}\right)_{N}\right)\left(1-2\left(\varphi_{\mathrm{I}}\right)_{N}\right)^{2}}{\left(\varphi_{\mathrm{I}}\right)_{N}}+O(1 / N)
$$

and since $\zeta_{\infty}=\left(\varphi_{\mathrm{I}}\right)_{\infty}$, we have

$$
\frac{\left(1-\left(\varphi_{\mathrm{I}}\right)_{\infty}\right)^{3}}{\left(\varphi_{\mathrm{I}}\right)_{\infty}} \frac{\left(1-2 \zeta_{\infty}\right)^{2}}{\left(1-\zeta_{\infty}\right)^{2}}=\frac{\left(1-\left(\varphi_{\mathrm{I}}\right)_{\infty}\right)\left(1-2\left(\varphi_{\mathrm{I}}\right)_{\infty}\right)^{2}}{\left(\varphi_{\mathrm{I}}\right)_{\infty}}
$$


Then

$$
\begin{aligned}
& \frac{\left(1-\left(\varphi_{\mathrm{I}}\right)_{N}\right)\left(1-2\left(\varphi_{\mathrm{I}}\right)_{N}\right)^{2}}{\left(\varphi_{\mathrm{I}}\right)_{N}}-\frac{\left(1-\left(\varphi_{\mathrm{I}}\right)_{\infty}\right)\left(1-2\left(\varphi_{\mathrm{I}}\right)_{\infty}\right)^{2}}{\left(\varphi_{\mathrm{I}}\right)_{\infty}} \\
& \quad=\frac{\left(\left(\varphi_{\mathrm{I}}\right)_{\infty}-\left(\varphi_{\mathrm{I}}\right)_{N}\right)}{\left(\varphi_{\mathrm{I}}\right)_{N}\left(\varphi_{\mathrm{I}}\right)_{\infty}}\left(1-8\left(\varphi_{\mathrm{I}}\right)_{N}\left(\varphi_{\mathrm{I}}\right)_{\infty}+4\left(\varphi_{\mathrm{I}}\right)_{N}\left(\varphi_{\mathrm{I}}\right)_{\infty}\left(\left(\varphi_{\mathrm{I}}\right)_{N}+\left(\varphi_{\mathrm{I}}\right)_{\infty}\right)\right)
\end{aligned}
$$

However, $\lim _{N \rightarrow \infty}\left(1-8\left(\varphi_{\mathrm{I}}\right)_{N}\left(\varphi_{\mathrm{I}}\right)_{\infty}+4\left(\varphi_{\mathrm{I}}\right)_{N}\left(\varphi_{\mathrm{I}}\right)_{\infty}\left(\left(\varphi_{\mathrm{I}}\right)_{N}+\left(\varphi_{\mathrm{I}}\right)_{\infty}\right)\right)=1-8\left(\left(\varphi_{\mathrm{I}}\right)_{\infty}\right)^{2}+$ $8\left(\left(\varphi_{\mathrm{I}}\right)_{\infty}\right)^{3} \neq 0$, since $0<\left(\varphi_{\mathrm{I}}\right)_{\infty}<\frac{1}{2}$. Therefore, $(11)$ is proved. However, by $(8)$, the left-hand side of $(11)$ must be of the order of $1 / N$. This proves that the case $1 / N=o\left(\left(\varphi_{\mathrm{I}}\right)_{\infty}-\left(\varphi_{\mathrm{I}}\right)_{N}\right)$ is impossible. Therefore, $\left(\varphi_{\mathrm{I}}\right)_{\infty}-\left(\varphi_{\mathrm{I}}\right)_{N}=O(1 / N)$ and we obtain

$$
\sqrt{\operatorname{var}\left[\tilde{p}_{N}-\tilde{p}_{\infty}\right]} \propto \beta_{N} N-\frac{1}{d}=O\left(\frac{1}{N}\right)
$$

(4) By (8)

$$
\frac{\sigma_{z}^{2} \rho^{2}}{\tau_{E}} \frac{N}{N-1}=\frac{\left(1-\left(\varphi_{\mathrm{I}}\right)_{N}\right)^{3}}{\left(\varphi_{\mathrm{I}}\right)_{N}} \frac{\left(1-2 \zeta_{N}\right)^{2}}{\left(1-\zeta_{N}\right)^{2}}
$$

and, thus, by (5),

$$
\left(\beta_{N} N\right)^{2}=\frac{\tau_{E}^{2}}{\rho^{2}}\left(1-\left(\varphi_{\mathrm{I}}\right)_{N}\right)^{2} \frac{\left(1-2 \zeta_{N}\right)^{2}}{\left(1-\zeta_{N}\right)^{2}}=\frac{\tau_{E}^{2}}{\rho^{2}} \frac{\sigma_{z}^{2} \rho^{2}}{\tau_{E}} \frac{\left(\varphi_{\mathrm{I}}\right)_{N}}{1-\left(\varphi_{\mathrm{I}}\right)_{N}} \frac{N}{N-1}
$$

Similarly

$$
\frac{1}{d^{2}}=\frac{\tau_{E}^{2}}{\rho^{2}} \frac{\sigma_{z}^{2} \rho^{2}}{\tau_{E}} \frac{\left(\varphi_{\mathrm{I}}\right)_{\infty}}{1-\left(\varphi_{\mathrm{I}}\right)_{\infty}}
$$

So

$$
\begin{aligned}
\left(\beta_{N} N\right)-\frac{1}{d} & \propto\left(\beta_{N} N\right)^{2}-\frac{1}{d^{2}} \propto \frac{\left(\varphi_{\mathrm{I}}\right)_{N}}{1-\left(\varphi_{\mathrm{I}}\right)_{N}} \frac{1}{N-1}+\left(\frac{\left(\varphi_{\mathrm{I}}\right)_{N}}{1-\left(\varphi_{\mathrm{I}}\right)_{N}}-\frac{\left(\varphi_{\mathrm{I}}\right)_{\infty}}{1-\left(\varphi_{\mathrm{I}}\right)_{\infty}}\right) \\
& \propto \frac{\left(1-\left(\varphi_{\mathrm{I}}\right)_{\infty}\right)\left(\varphi_{\mathrm{I}}\right)_{N}}{N-1}+\left(\varphi_{\mathrm{I}}\right)_{N}-\left(\varphi_{\mathrm{I}}\right)_{\infty}
\end{aligned}
$$

Also recall that, as we have shown in part (3) above, we should have

$$
\beta_{N} N-\frac{1}{d} \propto 2\left(\left(\varphi_{\mathrm{I}}\right)_{\infty}-\left(\varphi_{\mathrm{I}}\right)_{N}\right)-\xi_{\mathrm{I}}+o\left(\frac{1}{N}\right)
$$

Let us assume that $\beta_{N} N-1 / d=o(1 / N)$. Then we should have $\lim _{N \rightarrow \infty} N\left(\left(\varphi_{\mathrm{I}}\right)_{\infty}-\left(\varphi_{\mathrm{I}}\right)_{N}\right)=$ $\left(1-\left(\varphi_{\mathrm{I}}\right)_{\infty}\right)\left(\varphi_{\mathrm{I}}\right)_{\infty}$ and $\lim _{N \rightarrow \infty} 2 N\left(\left(\varphi_{\mathrm{I}}\right)_{\infty}-\left(\varphi_{\mathrm{I}}\right)_{N}\right)=\lim _{N \rightarrow \infty} N \xi_{\mathrm{I}}$. By Lemma $5, N \xi_{\mathrm{I}}=$ $\left(\tau_{v}+\tau_{E}\right) /\left(\left(\tau_{v} / \varphi_{\mathrm{U}}\right)+\tau_{E}\right)$, therefore $\beta_{N} N-1 / d=o(1 / N)$ implies an equality

$$
2\left(1-\left(\varphi_{\mathrm{I}}\right)_{\infty}\right)\left(\varphi_{\mathrm{I}}\right)_{\infty}=\frac{\tau_{v}+\tau_{E}}{\tau_{v} /\left(\varphi_{\mathrm{U}}\right)_{\infty}+\tau_{E}}
$$


This equality holds only if

$$
\left(1-\left(\varphi_{\mathrm{I}}\right)_{\infty}\right)=\frac{\left(\tau_{v}+\tau_{E}\right) / 2}{\tau_{v}+\tau_{E}\left(\varphi_{\mathrm{U}}\right)_{\infty}}
$$

This condition can be rewritten as

$$
\tau_{E}\left(\varphi_{\mathrm{U}}\right)_{\infty}^{2}+\left(\tau_{v}-\tau_{E}\right)\left(\varphi_{\mathrm{U}}\right)_{\infty}+\left(\tau_{E}-\tau_{v}\right) / 2=0
$$

This equation on $\left(\varphi_{\mathrm{U}}\right)_{\infty}$ has a determinant of $\left(\tau_{v}-\tau_{E}\right)^{2}-2\left(\tau_{E}-\tau_{v}\right) \tau_{E}=\tau_{v}^{2}-\tau_{E}{ }^{2}$. Therefore, for the case of $\tau_{E}>\tau_{v}$, this equation does not have a solution and $\beta_{N} N-1 / d=o(1 / N)$ is impossible. Thus, we must have $\beta_{N} N-1 / d \propto 1 / N$ for the case when $\tau_{E}>\tau_{v}$. Therefore,

$$
\sqrt{\operatorname{var}\left[\tilde{p}_{N}-\tilde{p}_{\infty}\right]} \propto \beta_{N} N-\frac{1}{d} \propto \frac{1}{N}
$$




\section{References}

[1] Admati, A. (1985) "A noisy rational expectations equilibrium for multi-asset security models," Econometrica 53, 629-657.

[2] Biais, B., D. Martimort and J.C. Rochet (2000), "Competing Mechanisms in a Common Value Environment", Econometrica 68(4), 799-837.

[3] Cho, J.W. and M. Krishnan (2000) "Prices as aggregators of private information: evidence from S\&P 500 Futures data", Journal of Financial and Quantitative Analysis 35, $111-126$.

[4] Cripps, M. and J. Swinkels (2006), "Efficiency of Large Double Auctions", Econometrica 74, 47-92.

[5] García, D. and B. Urosevic (2008), "Noise and Aggregation of Information in Large Markets", WP UPF.

[6] Gong, J. and P. McAfee (1996), "The General Double Auction Mechanism", Advances in Applied Microeconomics, vol. 6, M. Baye ed., JAI Press, 63-96.

[7] Grossman, S.J. and J.E. Stiglitz (1980) "On the impossibility of the informationally efficient markets," American Economic Review 70, 393-408.

[8] Hellwig, M.F. (1980) "On the aggregation of information in competitive markets," Journal of Economic Theory 22, 477-498.

[9] Kyle, A.S. (1989) "Informed speculation with imperfect competition," Review of Economic Studies 56, 317-356.

[10] Leland, H.E. (1992) "Insider Trading: Should It Be Prohibited?", Journal of Political Economy 100, 859-887.

[11] Reny, P.J. and M. Perry (2006) "Toward a strategic foundation for rational expectations equilibrium," Econometrica 74, 1231-1269.

[12] Royden, H.L. (1968) Real Analysis, second edition, MacMillan: New York.

[13] Rustichini, A., M.A. Satterthwaite and S.R. Williams (1994). "Convergence to efficiency in a simple market with incomplete information,” Econometrica 62, 1041-1063.

[14] Verrecchia, R. (1982) "Information aggregation in a noisy rational expectations economy," Econometrica 50, 1415-1430.

[15] Vives, X. (1995) "Short-term investment and the information efficiency of the market," Review of Financial Studies 8, 125-160. 
[16] Vives, X. (2002), "Private information, strategic behavior, and efficiency in Cournot markets," Rand Journal of Economics 33, 361-376.

[17] Yosha, O. (1997) "Diversification and competition: financial intermediation in a large Cournot-Walras economy," Journal of Economic Theory 75, 64-88. 


\section{CESifo Working Paper Series}

for full list see www.cesifo-group.org/wp

(address: Poschingerstr. 5, 81679 Munich, Germany, office@cesifo.de)

2385 Marko Koethenbuerger, How Do Local Governments Decide on Public Policy in Fiscal Federalism? Tax vs. Expenditure Optimization, September 2008

2386 Ronald McKinnon and Gunther Schnabl, China's Exchange Rate Impasse and the Weak U.S. Dollar, September 2008

2387 Yan-Leung Cheung, Yin-Wong Cheung and Alan T.K. Wan, A High-Low Model of Daily Stock Price Ranges, September 2008

2388 Louis Eeckhoudt and Harris Schlesinger, Changes in Risk and the Demand for Saving, September 2008

2389 Carsten Hefeker and Blandine Zimmer, Uncertainty and Fiscal Policy in an Asymmetric Monetary Union, September 2008

2390 Jay Pil Choi and Byung-Cheol Kim, Net Neutrality and Investment Incentives, September 2008

2391 Marcel Gérard, Financing Bologna, the Internationally Mobile Students in European Higher Education, September 2008

2392 Annette Alstadsæter and Knut Reidar Wangen, Corporations' Choice of Tax Regime when Transition Costs are Small and Income Shifting Potential is Large, September 2008

2393 António Afonso and Christophe Rault, 3-Step Analysis of Public Finances Sustainability: the Case of the European Union, September 2008

2394 Betsey Stevenson and Justin Wolfers, Economic Growth and Subjective Well-Being: Reassessing the Easterlin Paradox, September 2008

2395 Bernhard Eckwert and Itzhak Zilcha, Private Investment in Higher Education: Comparing Alternative Funding Schemes, September 2008

2396 Øystein Foros, Hans Jarle Kind and Jan Yngve Sand, Slotting Allowances and Manufacturers' Retail Sales Effort, September 2008

2397 Mohammad Reza Farzanegan, Illegal Trade in the Iranian Economy: Evidence from a Structural Model, September 2008

2398 Olivier Bos, Charity Auctions for the Happy Few, September 2008

2399 Robert S. Chirinko and Debdulal Mallick, The Marginal Product of Capital: A Persistent International Puzzle, September 2008 
2400 Ben D'Exelle and Arno Riedl, Elite Capture, Political Voice and Exclusion from Aid: An Experimental Study, September 2008

2401 Torben M. Andersen and Joydeep Bhattacharya, On Myopia as Rationale for Social Security, September 2008

2402 Fabienne Llense, French CEO Compensations: What is the Cost of a Mandatory Upper Limit?, September 2008

2403 Valentina Bosetti, Carlo Carraro, Alessandra Sgobbi and Massimo Tavoni, Delayed Action and Uncertain Targets. How Much Will Climate Policy Cost?, September 2008

2404 Robert G. Chambers, Rolf Färe, Shawna Grosskopf and Michael Vardanyan, Generalized Quadratic Revenue Functions, September 2008

2405 Leonidas Enrique de la Rosa, Overconfidence in a Career-Concerns Setting, September 2008

2406 Marcus Drometer and Johannes Rincke, The Design of Political Institutions: Electoral Competition and the Choice of Ballot Access Restrictions in the United States, September 2008

2407 Markku Lanne and Helmut Lütkepohl, Stock Prices and Economic Fluctuations: A Markov Switching Structural Vector Autoregressive Analysis, September 2008

2408 Thomas L. Brewer, International Energy Technology Transfers for Climate Change Mitigations, September 2008

2409 Alexander Kemnitz, Native Welfare Losses from High Skilled Immigration, September 2008

2410 Xavier Vives, Strategic Supply Function Competition with Private Information, September 2008

2411 Fabio Padovano and Roberto Ricciuti, The Political Competition-Economic Performance Puzzle: Evidence from the OECD Countries and the Italian Regions, September 2008

2412 Joan Costa-Font and Mireia Jofre-Bonet, Body Image and Food Disorders: Evidence from a Sample of European Women, September 2008

2413 Thorsten Upmann, Labour Unions - To Unite or to Separate?, October 2008

2414 Sascha O. Becker and Ludger Woessmann, Luther and the Girls: Religious Denomination and the Female Education Gap in $19^{\text {th }}$ Century Prussia, October 2008

2415 Florian Englmaier and Stephen Leider, Contractual and Organizational Structure with Reciprocal Agents, October 2008 
2416 Vittorio Daniele and Ugo Marani, Organized Crime and Foreign Direct Investment: The Italian Case, October 2008

2417 Valentina Bosetti, Carlo Carraro, Alessandra Sgobbi and Massimo Tavoni, Modelling Economic Impacts of Alternative International Climate Policy Architectures. A Quantitative and Comparative Assessment of Architectures for Agreement, October 2008

2418 Paul De Grauwe, Animal Spirits and Monetary Policy, October 2008

2419 Guglielmo Maria Caporale, Christophe Rault, Robert Sova and Anamaria Sova, On the Bilateral Trade Effects of Free Trade Agreements between the EU-15 and the CEEC-4 Countries, October 2008

2420 Yin-Wong Cheung and Daniel Friedman, Speculative Attacks: A Laboratory Study in Continuous Time, October 2008

2421 Kamila Fialová and Ondřej Schneider, Labour Market Institutions and their Effect on Labour Market Performance in the New EU Member Countries, October 2008

2422 Alexander Ludwig and Michael Reiter, Sharing Demographic Risk - Who is Afraid of the Baby Bust?, October 2008

2423 Doina Maria Radulescu and Michael Stimmelmayr, The Welfare Loss from Differential Taxation of Sectors in Germany, October 2008

2424 Nikolaus Wolf, Was Germany ever United? Evidence from Intra- and International Trade 1885 - 1933, October 2008

2425 Bruno S. Frey, David A. Savage and Benno Torgler, Noblesse Oblige? Determinants of Survival in a Life and Death Situation, October 2008

2426 Giovanni Facchini, Peri Silva and Gerald Willmann, The Customs Union Issue: Why do we Observe so few of them?, October 2008

2427 Wido Geis, Silke Uebelmesser and Martin Werding, Why go to France or Germany, if you could as well go to the UK or the US? Selective Features of Immigration to four major OECD Countries, October 2008

2428 Geeta Kingdon and Francis Teal, Teacher Unions, Teacher Pay and Student Performance in India: A Pupil Fixed Effects Approach, October 2008

2429 Andreas Haufler and Marco Runkel, Firms' Financial Choices and Thin Capitalization Rules under Corporate Tax Competition, October 2008

2430 Matz Dahlberg, Heléne Lundqvist and Eva Mörk, Intergovernmental Grants and Bureaucratic Power, October 2008

2431 Alfons J. Weichenrieder and Tina Klautke, Taxes and the Efficiency Costs of Capital Distortions, October 2008 
2432 Andreas Knabe and Ronnie Schöb, Minimum Wage Incidence: The Case for Germany, October 2008

2433 Kurt R. Brekke and Odd Rune Straume, Pharmaceutical Patents: Incentives for R\&D or Marketing?, October 2008

2434 Scott Alan Carson, Geography, Insolation, and Institutional Change in $19^{\text {th }}$ Century African-American and White Stature in Southern States, October 2008

2435 Emilia Del Bono and Daniela Vuri, Job Mobility and the Gender Wage Gap in Italy, October 2008

2436 Marco Angrisani, Antonio Guarino, Steffen Huck and Nathan Larson, No-Trade in the Laboratory, October 2008

2437 Josse Delfgaauw and Robert Dur, Managerial Talent, Motivation, and Self-Selection into Public Management, October 2008

2438 Christian Bauer and Wolfgang Buchholz, How Changing Prudence and Risk Aversion Affect Optimal Saving, October 2008

2439 Erich Battistin, Clara Graziano and Bruno Parigi, Connections and Performance in Bankers' Turnover: Better Wed over the Mixen than over the Moor, October 2008

2440 Erkki Koskela and Panu Poutvaara, Flexible Outsourcing and the Impacts of Labour Taxation in European Welfare States, October 2008

2441 Marcelo Resende, Concentration and Market Size: Lower Bound Estimates for the Brazilian Industry, October 2008

2442 Giandomenico Piluso and Roberto Ricciuti, Fiscal Policy and the Banking System in Italy. Have Taxes, Public Spending and Banks been Procyclical in the Long-Run? October 2008

2443 Bruno S. Frey and Katja Rost, Do Rankings Reflect Research Quality?, October 2008

2444 Guglielmo Maria Caporale, Antoaneta Serguieva and Hao Wu, Financial Contagion: Evolutionary Optimisation of a Multinational Agent-Based Model, October 2008

2445 Valentina Bosetti, Carlo Carraro and Massimo Tavoni, Delayed Participation of Developing Countries to Climate Agreements: Should Action in the EU and US be Postponed?, October 2008

2446 Alexander Kovalenkov and Xavier Vives, Competitive Rational Expectations Equilibria without Apology, November 2008 